\title{
Observation of the Dipole-Dipole Interaction in Dressed State Rydberg Atoms by Microwave Spectroscopy
}

\author{
Lauren Levac \\ Merton, WI
}

B.S., University of Wisconsin, 2010

A Thesis presented to the Graduate Faculty of the University of Virginia in Candidacy for the Degree of Masters of Science

Department of Physics

University of Virginia

May, 2013

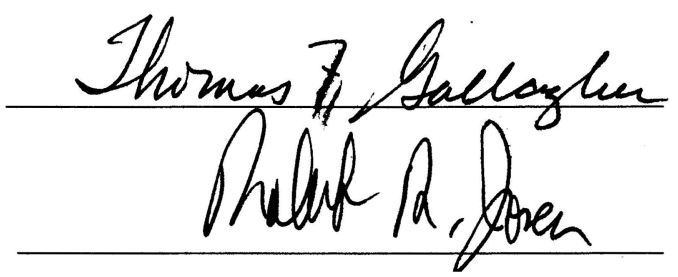




\begin{abstract}
Cold Rydberg atoms are a useful medium to study physical properties for several reasons. The trapped and cooled atoms move on the order of $0.24 \mu \mathrm{m} / \mu \mathrm{s}$ - essentially stationary on the timescale of our experiments. In addition Rydberg Rubidium atoms have one loosely bound electron at a distance from the ion core $\propto n^{2}$. When a dressing field is applied to that electron, it oscillates between states creating a single atom dipole which is a useful object of study. This thesis presents experimental studies of dressed state Rydberg atoms. It was found that the dressed state is highly sensitive to the polarization of the dressing field. We characterized dressed state cold Rydberg atoms - how they behave under changes in dressing frequency, dressing power, polarization of dressing and probe fields, and density.
\end{abstract}




\section{Contents}

1 Introduction 1

1.1 Dressed States of Rydberg Atoms . . . . . . . . . . . . . . . . . . . . . 1

1.2 Thesis Outline . . . . . . . . . . . . . . . . . . . . 3

2 Experimental Approach $4 \ldots$

2.1 Magneto-Optical Trap $(\mathrm{MOT}) \ldots \ldots \ldots \ldots \ldots \ldots \ldots \ldots$

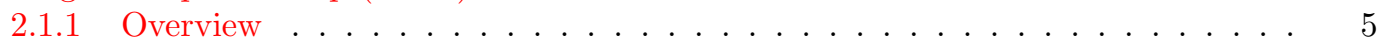

2.1.2 Littrow External Cavity Diode Laser . . . . . . . . . . . . . . . . . . 7

2.1.3 Frequency Locking Lasers via Saturated Absorption . . . . . . . . . . . . . . 9

2.1.4 Magnetic Fields . . . . . . . . . . . . . . . . . . . . . . . . . . . . . 13

Anti-Helmholtz Coils . . . . . . . . . . . . . . . . . . . . . . 13

Switching the MOT Magnetic Field . . . . . . . . . . . . . . 14

MOT Magnetic Field Circuit . . . . . . . . . . . . . . 15

Nulling Coils . . . . . . . . . . . . . . . . . . . . . . . 19

2.1.5 Ultra High Vacuum Chamber . . . . . . . . . . . . . . . . . . . . . . 19

2.2 Exciting and Detecting Rydberg Atoms . . . . . . . . . . . . . . . . . . . . 19

2.2 .1 Blue Laser . . . . . . . . . . . . . . . . . . . . . . . . 20

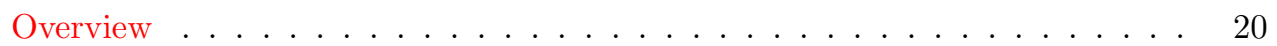

Toptica diode Laser . . . . . . . . . . . . . . . . . . . . . . . . 21

Tapered Amplifier . . . . . . . . . . . . . . . . . . . . . 21

Dye Amplification Stages and Frequency Doubling Crystal . . . . . . . . . 22

Triggering the Nd:YAG laser . . . . . . . . . . . . . . . . . . . 24

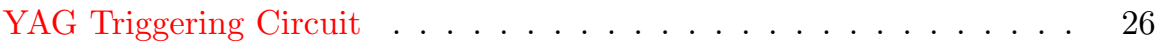

2.2 .2 Detecting the Rydberg Signal _ . . . . . . . . . . . . . . . . . . . . 29

Field Ionization Circuit _. . . . . . . . . . . . . . . . 31

2.3 Microwave Spectroscopy . . . . . . . . . . . . . . . . . . . . . . . 34

2.4 System Parameters . . . . . . . . . . . . . . . . . . . . . . 36

2.4.1 The Number of Atoms in the MOT and in the Rydberg state. . . . . . . . . 36

2.4.2 Calibrating Micro-Channel Plate Detector . . . . . . . . . . . . . . . . . . 39

2.4 .3 Width of MOT . . . . . . . . . . . . . . . . . . . 41

2.4 .4 Density . . . . . . . . . . . . . . . . . . . . 43

Density of MOT . . . . . . . . . . . . . . . . . 43

Density of Rydberg atoms . . . . . . . . . . . . . . . . . . . 44

2.4 .5 MOT Rise Time . . . . . . . . . . . . . . . . . . . . . 44

2.5 Nulling Stray Fields . . . . . . . . . . . . . . . . . . . . . . . 47

2.5 .1 Motivation . . . . . . . . . . . . . . . . . 47

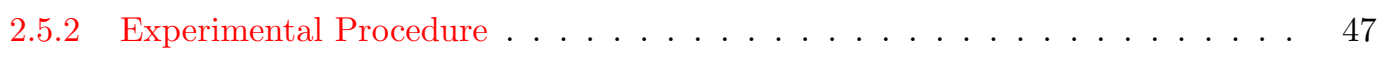

2.5 .3 Troubleshooting . . . . . . . . . . . . . . . . . 51 
3 Dressed State Experiment $\quad \mathbf{5 4}$

3.1 Introduction . . . . . . . . . . . . . . . . . . . . . . . . . . 54

3.2 Dressed State Theory . . . . . . . . . . . . . . . . . . . . . . . 54

3.2.1 Floquet Theory . . . . . . . . . . . . . . . . . . . 58

3.2.2 Calculating the Matrix Elements . . . . . . . . . . . . . . . . . . . 62

3.2.3 Calculating the Reduced Matrix Element . . . . . . . . . . . . . . . . . . . 64

3.2.4 Calculating the Hamiltonian's Eigenvalues . . . . . . . . . . . . . . . . . 65

3.3 Experimental Approach . . . . . . . . . . . . . . . . . . . . . . 69

3.4 Results and Analysis . . . . . . . . . . . . . . . . . . . . . . . 70

3.4.1 Polarization of the Fields . . . . . . . . . . . . . . . . . . . 71

3.4.2 Position Dependence . . . . . . . . . . . . . . . . . . . . . . . . . . . . . . . . . . . .

3.4.3 Dressing Field Amplitude Dependence . . . . . . . . . . . . . . . . . . . 78

3.4 Detuning dressed frequency . . . . . . . . . . . . . . . . . . . 79

3.4.5 Density Dependence ........................ 79

3.4.6 Relating Peak Frequency to Dressing Field Magnitude and Polarization . . . 85

4 Microwave Scattering Theory $\quad 91$

4.1 Modeling the Microwave EM Field Scattering from the Field Ionization Rods . . . . 91

4.2 Cylindrical Wave Equations . . . . . . . . . . . . . . . . . . . . . . . . . . 92

4.2.1 Scalar Wave Equation in Cylindrical Coordinates . . . . . . . . . . . . . . . 93

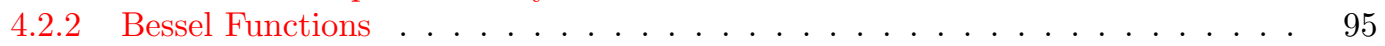

4.2 .3 Cylindrical Scalar Fields . . . . . . . . . . . . . . . . . . . . 98

4.2 .4 Vector Cylindrical Wave Functions . . . . . . . . . . . . . . . . . . . . 98

4.2 .5 Plane Waves in Cylindrical Wave Functions . . . . . . . . . . . . . . . . . . 99

4.3 Scattering from One Cylinder . . . . . . . . . . . . . . . . . . . 101

4.4 Scattering from Four Cylindrical Rods . . . . . . . . . . . . . . . . . . . . . . . 109

4.5 Polarization of the Field across the Finite MOT . . . . . . . . . . . . . . . . . . . 114

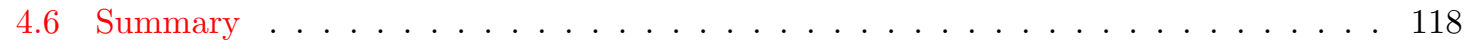

$\begin{array}{llr}5 & \text { Conclusions } & 120\end{array}$ 


\section{List of Figures}

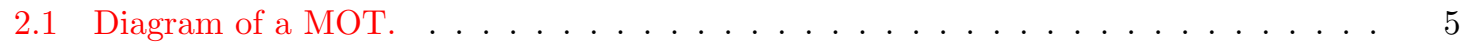

2.2 Energy levels used to trap Rubidium in a MOT. . . . . . . . . . . . . . . . 6

2.3 Diagram of Littrow configuration external cavity diode laser . . . . . . . . . . . . . 9

2.4 Saturated Absorption setup as used by repump and trapping lasers. . . . . . . . . . 10

2.5 Saturated Absorption Graphs . . . . . . . . . . . . . . . . . . . . . . . 10

2.6 Stick diagram of energy splittings of the allowed hyperfine transitions and the 'crossover

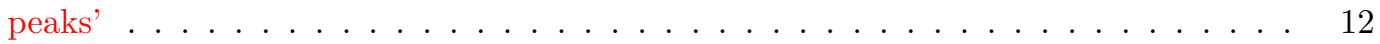

2.7 Diagram of MOT magnetic coils with coordinate system used in equations 2.1 and 2.2. 14

2.8 MOT magnetic field shut off . . . . . . . . . . . . . . . . . . . . . 15

2.9 Different MOT magnetic field switching delay times . . . . . . . . . . . . . . 16

2.10 Circuit used to control magnetic field for MOT. Component values in Table 2.3. .17

2.11 Diagram of Blue laser . . . . . . . . . . . . . . . . . . . . . . . . . . . 20

2.12 Diagram of tapered amplifier's alignment components . . . . . . . . . . . . . . 22

2.13 Power fluctuations in the YAG . . . . . . . . . . . . . . . . . . . . . . . . . . . . . . . . . . . . . .

2.14 YAG Triggering Circuit . . . . . . . . . . . . . . . . . . . . . . 27

2.15 Field Ionization Pulse Diagrams. . . . . . . . . . . . . . . . . . . . . . . 29

2.16 Graph of Field Ionization Pulse . . . . . . . . . . . . . . . . . . . . . . . . 30

2.17 Field Ionization Pulse Circuit $(\mathrm{FI})$. . . . . . . . . . . . . . . . . . . . . . . . . 32

2.18 FI pulse damping . . . . . . . . . . . . . . . . . . . . . . . . . . 33

2.19 MCP Circuit . . . . . . . . . . . . . . . . . . . . . . . 34

2.20 Setups for the two microwave fields used in this thesis. . . . . . . . . . . . . 35

2.2141 p transition . . . . . . . . . . . . . . . . . . . . . . . 37

2.22 Oscilloscope scan of MCP with a small 41p signal and a large 41s signal . . . . . . 40

2.23 MOT. . . . . . . . . . . . . . . . . . . . . . . . . 42

2.24 MOT filling time for different getter currents . . . . . . . . . . . . . . . . . . 46

2.25 Diagram of energy levels for nulling experiment . . . . . . . . . . . . . . . . . . 48

2.26 Timing diagram for nulling experiment . . . . . . . . . . . . . . . . . . . 48

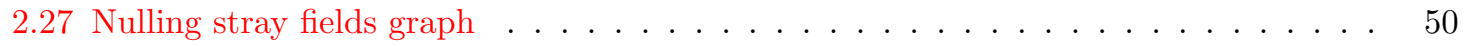

2.28 Field Ionization pulse overlap . . . . . . . . . . . . . . . . . . . . . . . . 52

2.29 Microwave power transition broadening . . . . . . . . . . . . . . . . 53

3.1 Diagram of the levels coupled in the dressed state . . . . . . . . . . . . . . . 55

3.2 Graphs of the off resonance eigenvalues . . . . . . . . . . . . . . . . . . 67

3.3 Graph of positive eigenvalues as a function of $\mathrm{g}$. . . . . . . . . . . . . . . 68

3.4 Transitions used in the Dressed State Experiment . . . . . . . . . . . . . . . . . . . 69

3.5 Timing diagram for the Dressed State Experiment . . . . . . . . . . . . . . . 70

3.6 Graph comparing horizontal dressing polarization with vertical dressing polarization. 72

3.7 Graph comparing horizontal probe polarization with vertical probe polarization $\quad$. 73 
3.8 Graph comparing two scans both with horizontal dressing and probe polarizations taken four hours apart. . . . . . . . . . . . . . . . . . . . 74

3.9 Diagram showing the normal orientation of microwave horns. . . . . . . . . . . 75

3.10 Graphs of shifting the dressing horn position with the dressing field polarization horizontal. . . . . . . . . . . . . . . . . . . . . 76

3.11 Graphs of shifting the dressing horn position with the dressing field polarization vertical. 77

3.12 Scanning over different field strengths, dressing field and probe field with horizontal polarization. . . . . . . . . . . . . . . . . . . . 80

3.13 Scanning over different field strengths, dressing field with vertical polarization and probe field with horizontal polarization. . . . . . . . . . . . . . . . . 81

3.14 Scanning the dressing field frequency. . . . . . . . . . . . . . . . . . 82

3.15 Graph of the off resonance eigenvalues for a linearly polarized interaction field. . . . 83

3.16 Low and high density MW scans. . . . . . . . . . . . . . . . . . . . 84

4.1 Diagram of $\mathrm{k}$ and its components. . . . . . . . . . . . . . . . . . 94

4.2 Diagram of a cylinder with an incoming plane wave . . . . . . . . . . . . 107

4.3 Diagram of actual set up drawn roughly to scale . . . . . . . . . . . . . . . 110

4.4 Diagram of the 4 field ionization rods with a perpendicular initial field. . . . . . . . 111

4.5 Diagram of the 4 field ionization rods with a tilted initial field. . . . . . . . . . . . 112

4.6 Diagram of points measured to get an idea of the variation over the size of the MOT. 115

4.7 These graphs depict the polarization of the field at different positions over a period. 117 


\section{List of Tables}

2.1 Theoretical wavelengths and frequencies for transitions used in this thesis. . . . . . 7

2.2 Typical values for various parts of the setup. . . . . . . . . . . . . . . . . 7

2.3 Components in MOT Magnetic Field Switching Circuit . . . . . . . . . . . . . . . . 18

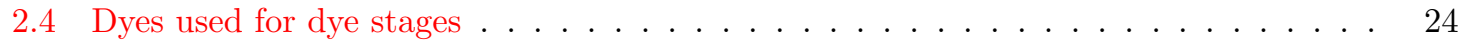

2.5 Components in YAG Triggering Circuit . . . . . . . . . . . . . . . . . . 28

2.6 Components in Field Ionization Circuit . . . . . . . . . . . . . . . . . . . . . . 32

2.7 Components in MCP Circuit . . . . . . . . . . . . . . . . . . . . . . . . . 32

2.8 Calibration values for MCP taken $9 / 30 / 11$ with $V_{M C P}=1648 \mathrm{~V}$ and $V_{F I}=40 \mathrm{~V} . \quad . \quad 41$

2.9 Widths of the MOT taken on different days . . . . . . . . . . . . . . . . . . . . 41

2.10 Summary of filling time of the MOT . . . . . . . . . . . . . . . . . . 45

2.11 Final nulling currents used to null all stray fields. . . . . . . . . . . . . . . . . . . . 49

2.12 Different types of microwave transition broadening and other problems . . . . . . . 51

3.1 Summary of Eigenvalues and degeneracies for different cases. . . . . . . . . . . . . 66

3.2 Horizontal polarization, experimental dressing field . . . . . . . . . . . . . . . . . 88

3.3 Vertical polarization, experimental dressing field . . . . . . . . . . . . . . . . . . 88

3.4 Horizontal polarization, statistics for $g, E, E_{d}$, and $P$ for Table 3.2 . . . . . . . 89

3.5 Vertical polarization, statistics for $g, E, E_{d}$, and $P$ for Table 3.3 . . . . . . . . 89

4.1 Coordinates for points in Figure 4.2 . . . . . . . . . . . . . . . . . . . . . . . . 107

4.2 Microwave field values in Cartesian coordinates at the center of the MOT . . . . . 114

4.3 Summarizes $g$ and $\phi$ for the total field at different points in the MOT. . . . . . . . . 119 


\section{Chapter 1}

\section{Introduction}

\subsection{Dressed States of Rydberg Atoms}

Rydberg atoms are an interesting system used to study a broad range of quantum phenomena [1]. Rydberg atoms are atoms where a electron is excited to a high energy level - n=14 to 200 or more. In the work described in this thesis we have used $n=41$. Since the radius of the electron's orbit is $\propto n^{2}$ these orbits quickly become large. The work done in this thesis was with Rubidium (Rb), an alkali atom. Since alkali atoms have only one valance electron, when it is excited to a Rydberg state the atom has a valence electron and ionic core and can be treated as being similar to Hydrogen. In addition, the binding energy of the electron to the atom is $\propto n^{-2}$ so these atoms are also loosely bound. The radiative lifetime scales as $n^{3}$. These properties make Rydberg atoms very useful for studying the interactions between atoms and external fields. One example of this Field Ionization, described in Section 2.2.2. Another is the dipole-dipole interaction, and studying it is the ultimate goal of the dressed state experiment, described in Chapter 3.

While the large principle quantum number has advantages, it can also present challenges. The energy gap between adjacent levels decreases as $n^{-3}$. Therefore, high resolution excitation lasers are necessary to selectively excite the electrons to a specific Rydberg energy level. This has been done using a diode laser amplified through dye cells as described in Section 2.2.1. In addition, it is possible to change Rydberg states with high resolution microwave fields. For example, the transition from $41 s_{1 / 2} \rightarrow 41 p_{1 / 2}$ is used as a probe of other interactions in this work with extremely high accuracy. 
The Rb atoms can be trapped using a magneto-optical trap (MOT), which confines and cools the atoms to $300 \mu \mathrm{K}$. At that temperature they move only $0.24 \mu \mathrm{m}$ in $1 \mu \mathrm{s}$ [2] and can be referred to as a frozen Rydberg gas. Since the time scale of interest is microseconds, the atoms are effectively stationary-and therefore atom-atom interaction does not change over the course of the experiment. Moreover, by switching off the trapping magnetic field and nulling any other stray fields (see Section 2.5), any dephasing effects from inhomogeneous magnetic fields can be minimized.

Dressed state atoms are created by coupling two states with a microwave pulse. In this experiment $n p$ and $(n+1)$ s states were coupled. This coupling causes the eigenstates to become a mixture of $n p$ and $(n+1)$ s. The eigenvalues are derived in Section 3.2. This phenomena can be viewed through microwave spectroscopy, as detailed in Section 3.3. The oscillation between states creates a dipole moment within the atom itself. This dipole moment can then interact with corresponding dipoles in nearby atoms. The dipole-dipole interaction is position and orientation dependent. By studying the interactions in a frozen Rydberg gas with dressed state atoms of different densities, we can learn more about them. Since dipole-dipole interactions are an important part of atom-atom interactions, it's study is useful for atomic and molecular systems in a number of scientific fields including quantum information $[3,4,5]$.

Studying dipole-dipole interactions was the original goal of this thesis. However as often happens in science, other phenomena previously unconsidered dominated the results. Microwave fields were used to create the dressed states and high resolution microwave spectroscopy was used to probe the states. It was found that the fields scatter and create a non-homogeneous elliptically polarized dressing field. This results in a loss of degeneracy in the eigenvalues - seen as broadening or additional splitting when the dressed levels are probed with a second, probe, microwave field.

This thesis describes the dressed state generated by fields with an elliptical component from scattering. While some aspects are setup dependent, it's useful to characterize this source of broadening and splitting in atomic resonances that can occur in high resolution microwave spectroscopy of dressed state atoms. 


\subsection{Thesis Outline}

The subsequent chapters describe 2 the experimental approach, 3 the dressed state experiment, 4 the microwave scattering theory, and 5 conclusions.

A large portion of the work done over the two years was in understanding, maintaining, and calibrating the experimental apparatus used for the experiments. Chapter 2 gives an outline of the various part of the set up used and some of the calibration measurements done. The first three of the five sections cover the three stages of the setup. First is the MOT, used to trap and cool the atoms. Next was exciting the atoms to Rydberg states and detecting what state they are in. Third we discuss using microwave spectroscopy to scan across a transition and learn about the atoms. The fourth section discusses some physical properties of the MOT. The final section covers a crucial and delicate part for the setup; nulling all stray fields, so they don't cause inhomogeneous broadening and obscure the information sought. There were no previous estimated values for the setup, and due to the limits of the current controllers for the nulling coils, it was incredibly sensitive. It was also incredibly educational, as most everything that could foul up any later experiment occurred during this experiment. This section also contains a brief troubleshooting table to help identify any problems that come up.

Chapter 3 outlines the dressed state experiment. The goal of this experiment was to understand more about how the dipole created by the dressed state behaves. However, it turns out the polarization of the dressing field plays a far larger role than expected initially. As a result, most of the section is on characterizing the atoms' behavior in the dressing field. This section starts with an introduction, followed by an experimental approach section. The experimental approach section outlines the details specific to that experiment, but builds on the information in the experimental approach chapter. Next is a section containing the results and analysis.

Chapter 4 outlines the theory for scattering a field off four cylindrical rods. The result of this chapter is a first order approximation of the total dressing field at the MOT under different initial conditions.

Chapter 5 discusses the conclusions that can be drawn from this experiment and suggests additional potential avenues to pursue. 


\section{Chapter 2}

\section{Experimental Approach}

Before conducting any new research, it is necessary to achieve useful experimental conditions in the setup and then maintain them daily. Since all components, barring the optical tables, decay in time this can be a time consuming process. In this chapter the various parts necessary for the experimental setup are described as well as some useful measurements of the magneto-optical trap (MOT). For more details, previous theses can be recommended. For example, Anderson's thesis [6] has an excellent theoretical and practical description of the MOT, but his work was done on a different setup. Wenhui's thesis was preformed on the same setup as this one and is a good source for details on it [7]. Hyunwook Park taught me a great deal about how to run the experimental apparatus and his thesis [2] contains more details on a number of procedures.

This chapter starts with outlining the experimental components in order of experimental procedure2.1 Magneto-Optical Trap (MOT), 2.2 Rydberg atoms, and 2.3 Microwave Spectroscopy - then proceeds to various measurements to characterize the MOT - 2.4 System Calibration - and finishes with 2.5 Nulling stray fields. Every day before any new experiment can be started, nulling all stray fields must be confirmed through measurement of a transform-limited microwave transition lineshape. 


\subsection{Magneto-Optical Trap (MOT)}

\subsubsection{Overview}

A Magneto-Optical Trap (MOT) is used to trap Rubidium atoms at temperatures around $300 \mu \mathrm{K}[7]$ in an ultra-high vacuum chamber. The MOT creates a potential well at the center of the vacuum chamber in both position and velocity space. There are two main components: the anti-Helmholtz magnetic coils and a trap laser. A repump laser is also necessary to maintain a MOT. They are diagrammed in Figure 2.1.

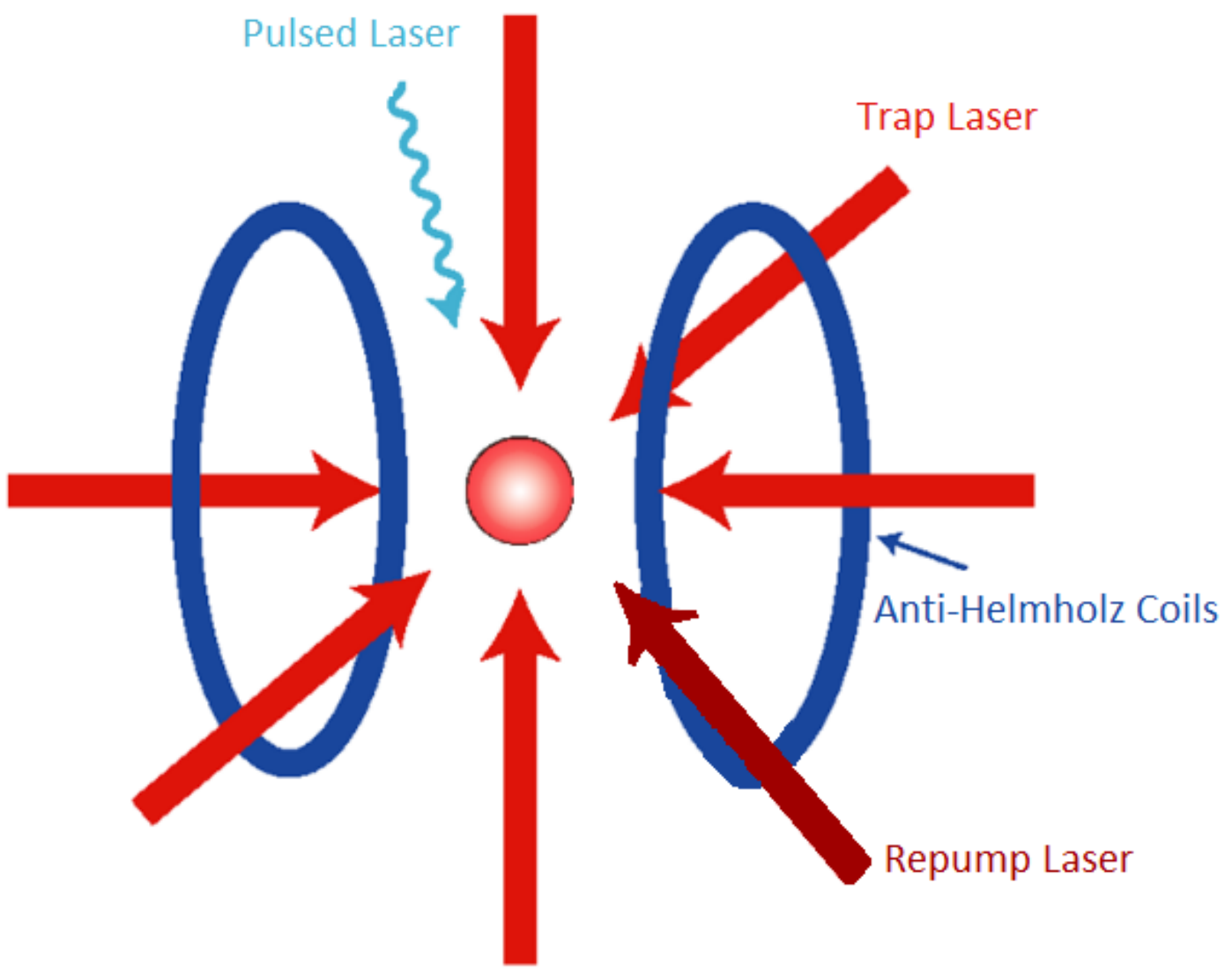

Figure 2.1: Diagram of a MOT.

The trap laser is split into three beams, sent through the chamber on three perpendicular axes, and then each beam is retro-reflected on itself. The repump laser is sent in on a fourth axis and 
intersects the other lasers at the center of the chamber. Trapping utilizes the $5 s_{1 / 2} \rightarrow 5 p_{3 / 2}$ transition shown in Figure 2.2. Rubidium atoms' valence electron's ground state is $5 s_{1 / 2}$ which has the hyperfine levels $\mathrm{F}=2$ and $\mathrm{F}=3$. The trap laser excites electrons from $5 s_{1 / 2} F=3 \rightarrow 5 p_{3 / 2} F=4$. However, the trap laser is red detuned a small amount from the transition. This mean that atoms that are moving in the opposite direction to a laser's propagation will be more likely to absorb a photon due to the first order Doppler shift. The photon is then re-emitted in a random direction. The absorption of multiple photons will give a net momentum kick, slowing the atom down and keeping it nearly at rest. This is referred to as "optical molasses."

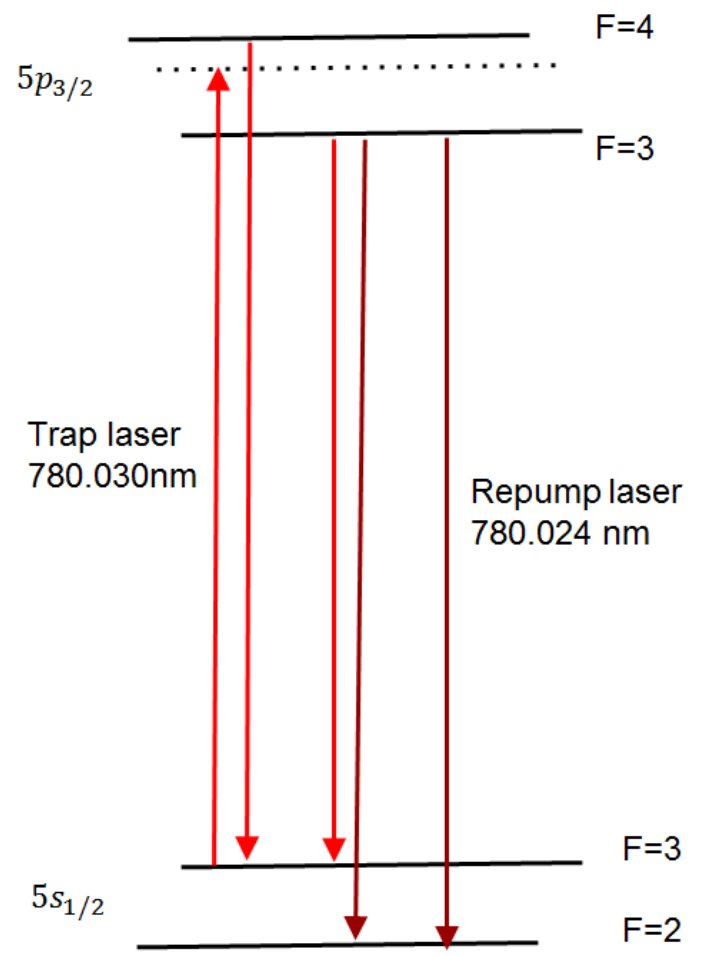

Figure 2.2: Energy levels used to trap Rubidium in a MOT.

The magnetic coils create a spatially dependent force pulling the atoms towards the center of the trap. They do this by producing a magnetic field which creates a Zeeman shift increasing with distance from the center of the trap. Since the trap laser is red detuned, the increase in the Zeeman shift means the atoms away from the center of the trap preferentially absorb a photon pushing them back towards the center of the chamber. This creates a potential well in position space.

The repump laser is necessary because there is a non-zero probability $(\sim 1 \%)$ that trap laser will 
excite an atom to the $5 p_{3 / 2} F=3$ state and it decays into $5 s_{1 / 2} F=2$ instead of $5 s_{1 / 2} F=3$ or the atoms may have originally been in the $5 s_{1 / 2} F=2$ state. Atoms in the $5 s_{1 / 2} F=2$ state will not be excited by the trap laser. The repump laser excites those atoms back to $5 p_{3 / 2} F=3$ so they have the chance to decay into $5 s_{1 / 2} F=3$ and see the trap laser.

These three components trap the atoms into a MOT. If any one of them is blocked or disrupted for longer than a couple milliseconds the trap will completely disperse.

The theoretical wavelengths (in air) and frequencies used in this thesis are listed in Table 2.1. Some components have frequency multiplicative elements and for them the initial frequency or wavelength is also given. Typical values for currents, temperatures and other settings are found in Table 2.2 with references to the section to where the component is explained.

\begin{tabular}{|l|l|l|l|l|}
\hline Initial State & Final State & Wavelength (in air) & Frequency & Value Pre-Multiplier \\
\hline $5 s_{1 / 2, F=3}$ & $5 p_{3 / 2, F=4}$ & $780.024 \mathrm{~nm}$ & & \\
\hline $5 s_{1 / 2, F=2}$ & $5 p_{3 / 2, F=3}$ & $780.030 \mathrm{~nm}$ & & \\
\hline $5 p_{1 / 2}$ & $41 s_{1 / 2}$ & $480.691 \mathrm{~nm}$ & & $961.382 \mathrm{~nm}$ \\
\hline $41 s_{1 / 2}$ & $41 p_{1 / 2}$ & & $56.629 \mathrm{GHz}$ & $14.1577 \mathrm{GHz}$ \\
\hline $41 p_{1 / 2}$ & $42 s_{1 / 2}$ & & $59.8966 \mathrm{GHz}$ & $19.9656 \mathrm{GHz}$ \\
\hline
\end{tabular}

Table 2.1: Theoretical wavelengths and frequencies for transitions used in this thesis.

\begin{tabular}{|l|l|l|l|}
\hline Component & part & Setting Value & Discussed in Section: \\
\hline \multirow{2}{*}{ Trap Laser } & Current & $73.7 \mathrm{~mA}$ & 2.1 .1 \\
\cline { 2 - 4 } & Temperature & $14 \mathrm{k} \Omega$ & 2.1 .1 \\
\hline \multirow{2}{*}{ Repump Laser } & Current & $63.5 \mathrm{~mA}$ & 2.1 .1 \\
\cline { 2 - 4 } & Temperature & $24.1^{\circ} \mathrm{C}$ & 2.1 .1 \\
\hline \multirow{2}{*}{960 nm Laser } & Current & $71.9 \mathrm{~mA}$ & 2.2 .1 \\
\cline { 2 - 4 } & Temperature & $22.9^{\circ} \mathrm{C}$ & 2.2 .1 \\
\hline \multirow{2}{*}{ Tapered Amplifier } & Current & $71.9 \mathrm{~mA}$ & 2.2 .1 \\
\cline { 2 - 4 } & Temperature & $22.9^{\circ} \mathrm{C}$ & 2.2 .1 \\
\hline Getter (supplies Rd Atoms) & Current & $4.7 \mathrm{~A}$ & 2.4 .5 \\
\hline Field Ionization & DC voltage & $30 \mathrm{~V}$ & 2.2 .2 \\
\hline MCP & Voltage & $1648 \mathrm{~V}$ & 2.2 .2 \\
\hline
\end{tabular}

Table 2.2: Typical values for various parts of the setup.

\subsubsection{Littrow External Cavity Diode Laser}

Stable, narrow linewidth diode lasers are an essential component of the MOT. The lasers excite atoms between different hyperfine levels. Due to the relatively small spacing between hyperfine levels and 
the necessity for precise red-detuning, the laser linewidth needs to be less than $5 \mathrm{MHz}$. This can be done with Littrow external cavity diode lasers which naturally have linewidths of $\sim 1 \mathrm{MHz}$. The challenge is maintaining a specific frequency. To solve that problem, we side lock the lasers to a saturated absorption peak, described in Section 2.1.3.

Both the trap and repump lasers are homemade Littrow configuration diode lasers. The diagram for this setup can be seen in Figure 2.3. The laser diode is temperature and current controlled by ILX Lightwave controllers. The laser is collimated by a short focal length lens placed at a distance equal to the focal length from the diode such that the beam stays approximately the same size for the entirety of its path. The diffraction grating and the length of the external cavity it creates are frequency selective elements. Changing the angle shifts the first order wavelength that is reflected back into the diode. The feedback from this light will pass through the laser's gain element multiple times and therefore be of greater intensity. The second frequency selective element is the cavity length. The cavity will have constructive interference for wavelengths where the length of the cavity is an integer multiple of half the wavelength. The diffraction grating position can be set coarsely using a adjustment screw and fine tuned by a piezoelectric stack (piezo). The piezo length can be controlled through an applied voltage from the locking circuit. Using the diode current and temperature it is possible to tune the lasers to the needed wavelengths. Key in tuning is patience and shifting mode hops (where the laser jumps in wavelength) away from the desired wavelength.

I have had success finding the correct wavelength by first using the wavemeter to observe whether I'm close to the correct wavelength, and then looking for the correct saturated absorption spectrum (SAS). First use the adjustment screw for the diffraction grating to get withing $\sim 0.05 \mathrm{~nm}$ of the desired wavelength. Next temperature and current can be manipulated to get the wavelength within $\sim 0.005 \mathrm{~nm}$ of the desired wavelength. However, the wavemeter has a slight offset that varies with time which needs to be taken into account. The piezo can scan over $\sim 0.01 \mathrm{~nm}$, and it should be possible to use it to find the right saturated absorption spectrum (SAS). Their most common appearance for my setup is pictured in Figure 2.5. If the laser mode-hops before the right frequency, try changing the temperature. Once you have the SAS, frequently it can be optimized by small changes to the current and peizo in tandem. The repump laser should be at $780.030 \mathrm{~nm}$, and the trap laser at $780.024 \mathrm{~nm}$. 


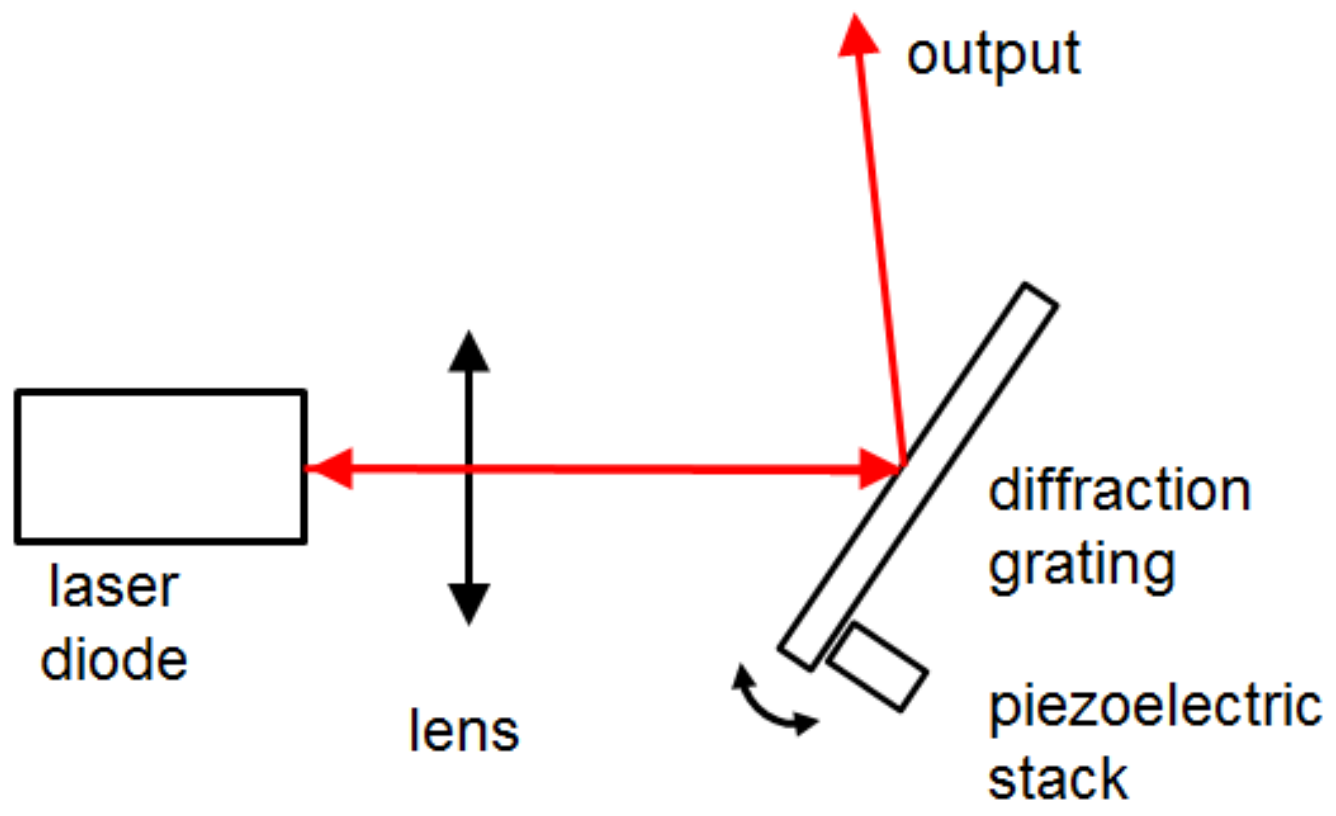

Figure 2.3: Diagram of Littrow configuration external cavity diode laser

\subsubsection{Frequency Locking Lasers via Saturated Absorption}

To trap atoms it is necessary for the trapping and repump lasers to be tuned to very specific wavelengths and to stay at those wavelengths. The lasers easily drift in frequency, because they are very sensitive to mechanical vibrations, temperature fluctuations, and current fluctuations. It is impossible to keep the lab silent and stable enough to hold the diode frequency constant, so this stability is accomplished through a feedback system.

A few percent of the laser beams are picked off and sent to a saturated absorption setup, pictured in Figure 2.4. Ramping the frequency of the laser generates a photodiode signal with hyperfine peaks. Figure 2.5 contains graphs for the transitions used to lock both the repump and trap lasers. A lock box can then be used to side-lock to a specific point on the hyperfine spectrum indicated by an arrow. Whenever the laser tries to drift off the chosen wavelength, the lock box will adjust the voltage sent to the piezo, changing the laser's cavity length and therefore frequency, and "push" the laser back to the correct place in the spectrum.

A saturated absorption setup (see Figure 2.4) sends the laser through a glass cell containing 


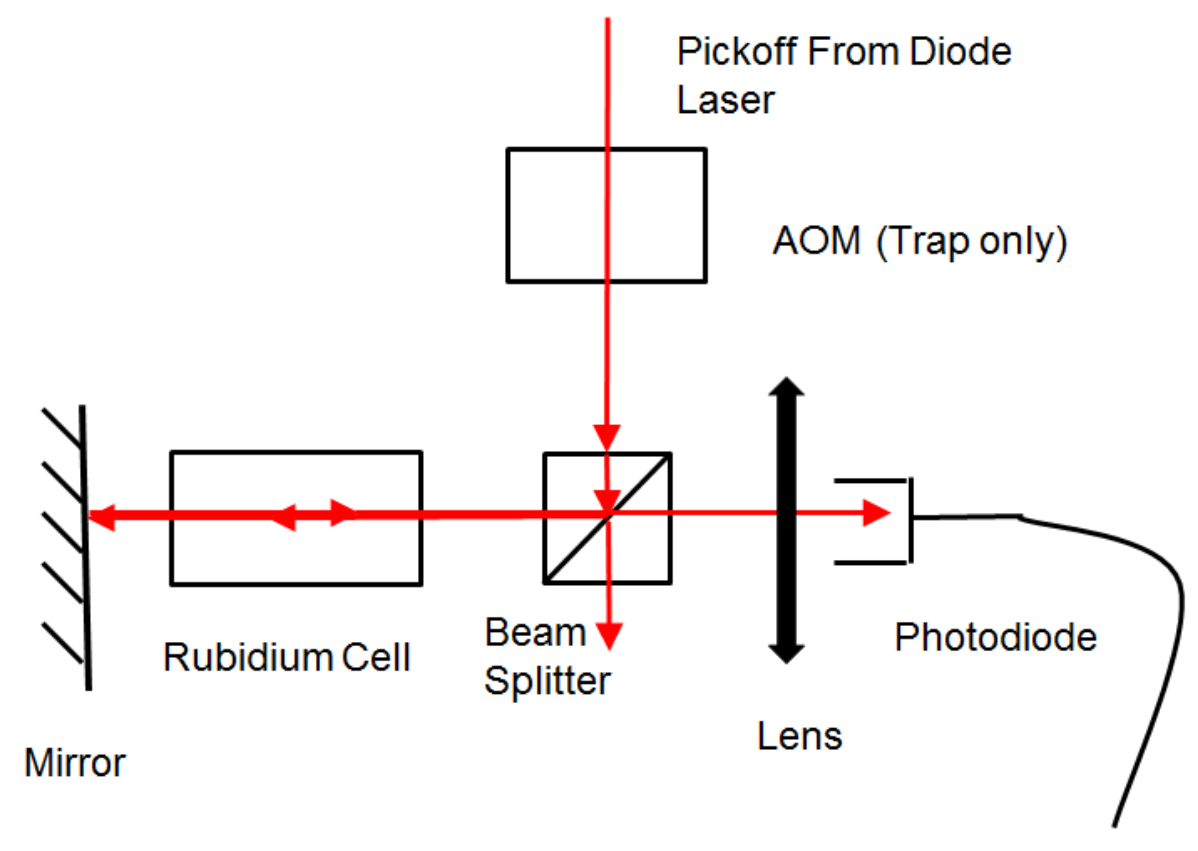

To Lock Box

Figure 2.4: Saturated Absorption setup as used by repump and trapping lasers.

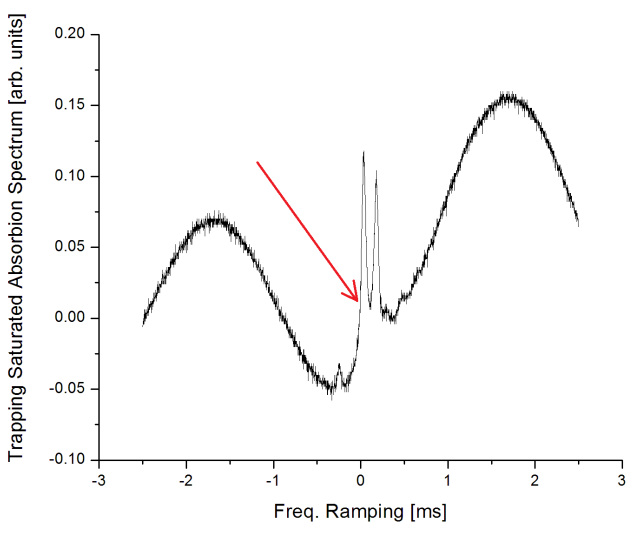

(a) Trap SAS, scans over $R b^{85} \mathrm{~F}=3-\mathrm{F}^{\prime}$.

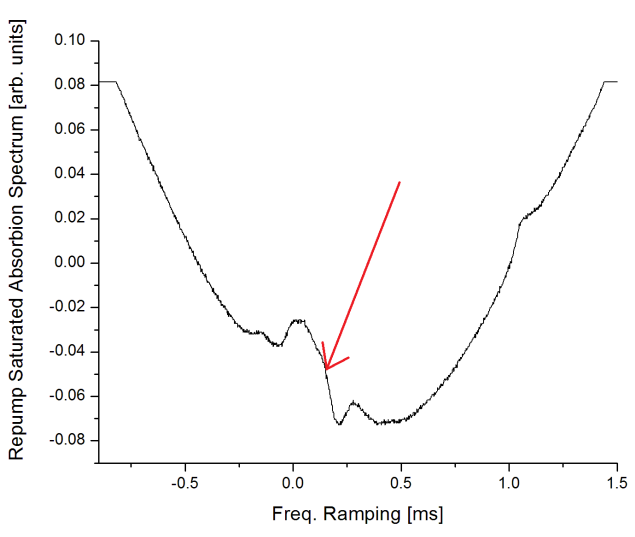

(b) Repump SAS, scans over $R b^{85} \mathrm{~F}=2-\mathrm{F}$ '.

Figure 2.5: Saturated Absorption Spectrum (SAS) used for locking both lasers. These are created using a ramped voltage sent to the piezo to ramp the laser over a range of frequencies. Frequency increases to the left. 
rubidium atoms. If a photo-diode were to be placed directly after this, the spectrum viewed through ramping the frequency would be Doppler broadened to about $1 \mathrm{GHz}$ wide. It would not be possible to distinguish the hyper-fine transitions. Instead, the laser is retro-reflected along its previous path through the cell. When it is retroreflected through the cell, the Doppler shift of the light relative to the motion of the atoms is opposite the shift on the first pass through the cell. The spectrum will then contain a Doppler broadened peak with several dips. These occur at the non-Doppler shifted transition frequencies and crossover frequencies. At these unshifted frequencies atoms not moving in the direction of the laser propagation have no Doppler shift, and the absorption from the first pass will diminish absorption on the second pass. Crossover frequencies are half way between two transition frequencies. The Doppler shifts of the two transitions cancel and there is a peak from the increased absorption. The cross over peaks are frequently large. See Anderson's thesis [6] for an excellent explanation of crossover peaks. Figure $2.6[6]$ shows a stick diagram of the allowed hyperfine transitions and the crossover peaks. It contains spectra for both $\mathrm{Rb}^{85}$ and $\mathrm{Rb}^{87}$, the two most common isotopes. In all experiments we use $\mathrm{Rb}^{85}$. Figure 2.5 shows the actual oscilloscope traces for the trap and repump lasers.

To align the saturated absorption setup shown in Figure 2.4, first pick off some light from the main beam. The light is sent at roughly 90 degree incidence to a beam splitter. The light is sent through the rubidium cell, then retro-reflected back on itself. The retro-reflection needs to be precise. An iris or a note card with a small hole is necessary for this. Finally a photo-diode (Thorlabs Det110) is placed in the path of the retro-reflected beam after it has gone through the beam splitter again. An optional lens placed before the photo diode makes the photo-diode's alignment less crucial. Small adjustments in the retro-reflecting laser direction can be used to maximize the size of the hyperfine structure.

Since the diode laser's frequency drifts easily, it's necessary to lock the laser to a specific frequency. The trap laser is side-locked to the high frequency side of the crossover peak of the $\mathrm{F}=3 \rightarrow 3$ and $\mathrm{F}=3 \rightarrow 4$ resonances. The crossover peak frequency is $60.4 \mathrm{MHz}$ less than the $\mathrm{F}=3 \rightarrow 4$ resonance and the point where it is side-locked is approximately $54.6 \mathrm{MHz}$ less. An arrow points to the location in Figure 2.5a. In between the laser pick-off and the beamsplitter directing the laser beam to the Rb cell the beam is sent through an acousto-optic modulator (AOM) from Intraaction corp (AOM-40N). The AOM shifts the frequency of the picked off light down by $42 \mathrm{MHz}$. This is equivalent to shifting 


\section{$\Delta \mathrm{F}=1$ Hyperfine Transitions}

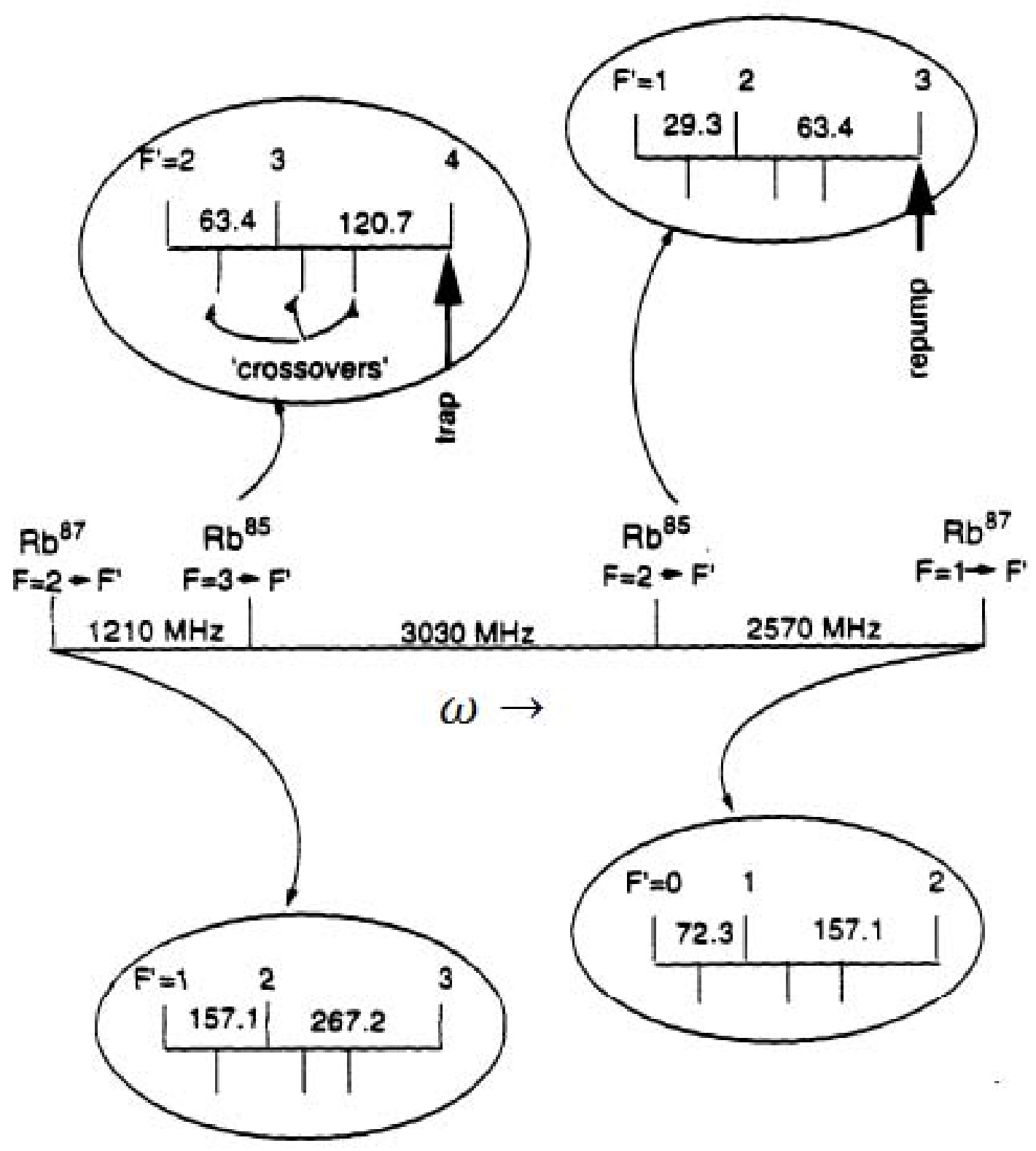

Figure 2.6: Stick diagram (not to scale) of energy splittings of the allowed hyperfine transitions and the 'crossover peaks' seen in the saturated absorption spectrum. Taken from Anderson [6]. 
the trap laser frequency $42 \mathrm{MHz}$ higher. This gives the trap laser a total detuning of $\sim 12 \mathrm{MHz}$ from the $\mathrm{F}=3 \rightarrow 4$ transition. The exact detuning is dependent on where the laser is side-locked on the slope of the crossover peak. This is optimized daily to produce the best red-shift for the MOT. The repump laser frequency is locked near to the $\mathrm{F}=2 \rightarrow 3$ resonance, indicated in Figure 2.5b by an arrow. Once the lasers are locked, potential frequency shifts are countered through the piezo shifting the diffraction grating in the laser cavity. The lasers can remain locked for more than an hour provided there are not any disturbances or vibrations.

\subsubsection{Magnetic Fields}

\section{Anti-Helmholtz Coils}

The anti-Helmholtz coils provide a gradient magnetic field over vacuum chamber (see Figure 2.1). This will be referred to elsewhere in the thesis as the MOT magnetic field. A brief theoretical discussion of its purpose is in Section 2.1.1. There are two equally sized rings wound 95 times with copper magnet wire with Polyurethane-Nylon coating in an aluminum housing. The coils are water cooled to prevent the wire insulation from melting. The coil rings are $2 R \cong 15 \mathrm{~cm}$ in diameter and separated by $2 A \cong 12 \mathrm{~cm}$. An equal but oppositely directed current is sent through each coil. This creates a field that cancels out at the center of the vacuum chamber (where the MOT will be) and increases away from it. The magnetic field near the axis of these coils can be expressed as [7]:

$$
\begin{aligned}
B_{z}=\sum_{n} b_{n} B_{z n} & =b_{i} z+b_{3}\left[z^{3}-3 z \rho^{2}\right]+\cdots \\
B_{\rho}=\sum_{n} b_{n} B_{\rho n} & =b_{i} z+b_{3}\left[-\frac{2 \rho z^{2}}{2}+\frac{\rho^{2} z}{8}\right]+\cdots \\
b_{1} & =\frac{3 \mu_{0} I A R^{2}}{\left(R^{2}+A^{2}\right)^{5 / 2}} \\
b_{2} & =\frac{5\left(4 A^{2}-3 R^{2}\right)}{6\left(A^{2}+r^{2}\right)} \times b_{1}
\end{aligned}
$$

where the coordinate system is defined with the origin at the center between the two coils, $\mathrm{z}$ is along the axis connecting the center of the coils, $\rho$ is the distance from the $\mathrm{z}$ axis, the coils are in the $z= \pm A$ planes, and $\mathrm{R}$ is the radius of the coils. This is diagrammed in Figure 2.7. For our case, 


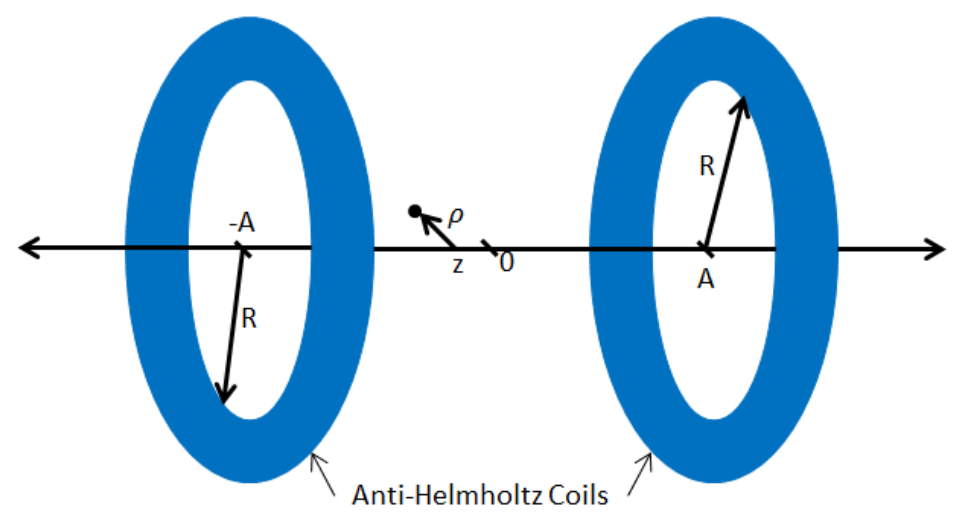

Figure 2.7: Diagram of MOT magnetic coils with coordinate system used in equations 2.1 and 2.2.

$\mathrm{A} / \mathrm{R} \approx 0.8$ so $b_{1} \gg b_{3}$. This means the field increases approximately linearly in all directions from zero field at the center of the of the pair of coils. The magnetic field gradients are[7]:

$$
\frac{\partial B}{\partial z}=-2 \frac{\partial B}{\partial \rho}=b_{1}=1.48 \times I(\text { Amp })(\text { Gauss } / \mathrm{cm})
$$

The coils can be run either in a non-switching mode or switching mode. Non-switching mode supplies a constant current (10A) to the coils creating a field gradient that is present at all times. Switching mode, described below, diverts the current from the coils for part of a $30 \mathrm{~Hz}$ cycle. During the brief time the coils are off it is possible to run experiments that are sensitive to external magnetic fields.

\section{Switching the MOT Magnetic Field}

Rydberg states are extremely sensitive to external fields, which is part of what makes them interesting to study. However, the MOT magnetic field used to trap the atoms is stronger than any external magnetic field that might be studied. Even more important is reducing the inhomogeneous broadening $(\sim 5 \mathrm{MHz})$ of the transition resonances. For this reason, it's necessary to switch off the MOT magnetic field when we are probing the atoms' interactions. Due to the large current used in the coils to create the MOT magnetic field and the aluminum coil housing, the field does not turn off instantaneously but falls off exponentially on the scale of milliseconds, as depicted in Figure 2.8. Figure 2.9 shows how the transition $5 p_{3 / 2} \rightarrow 41 s_{1 / 2}$ changes as a function of the delay time between 
switching off the magnetic field and triggering the blue laser pulse. For $5 \mathrm{~ms}$ and more of delay time before the blue pulse, there is no broadening due to stray fields from the MOT magnetic field. At $4 \mathrm{~ms}$, broadening starts to appear and becomes more apparent as the delay is decreased. With no delay, the transition line width is four times that of the transform-limited peak and shows definite signs of splitting from the inhomogeneous magnetic field. In all other experiments a $5 \mathrm{~ms}$ delay time was used. The coils are then switched back on $1 \mathrm{~ms}$ after the blue laser pulse, after the atoms have been ionized and the data collected (Section 2.2.2).

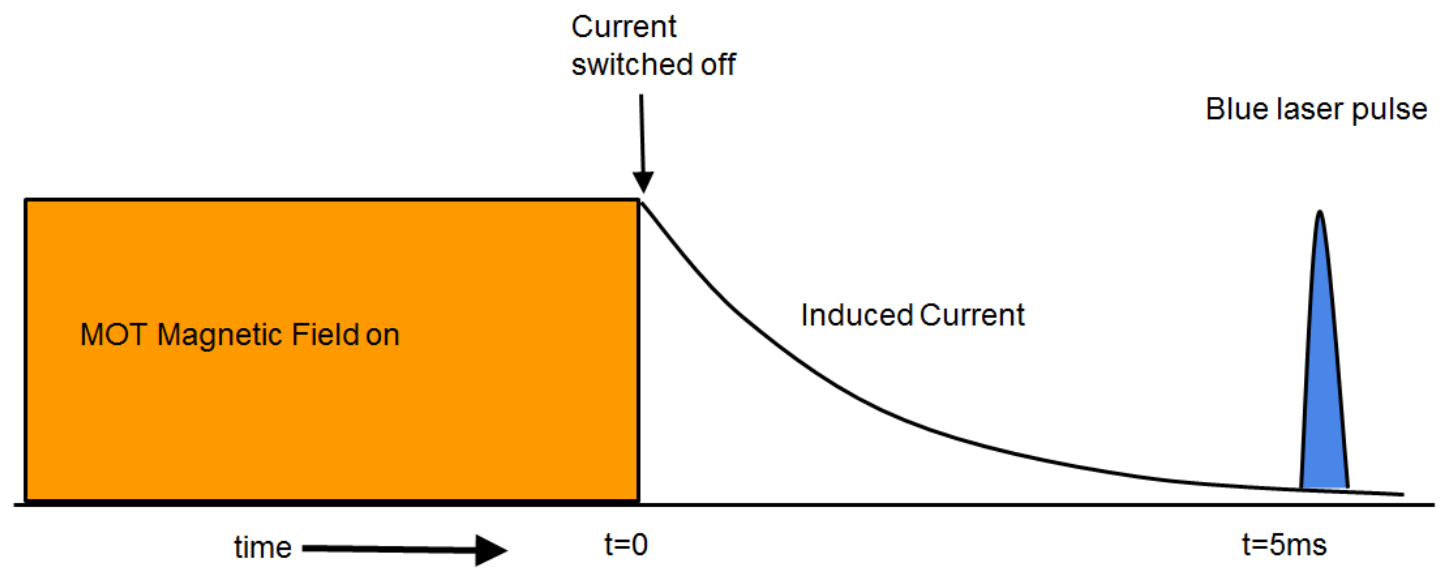

Figure 2.8: MOT magnetic field current turning off still leaves an induced current that needs to decay before the blue laser shot can excite the atoms without stray fields.

MOT Magnetic Field Circuit The magnetic field current is switched (or left constant) using the circuit in Figure 2.10 with the components listed in Table 2.3. There are two inputs into the circuit: $C O N N_{1}$ connects a pulse generator via a BNC cable to circuit, and $C O N N_{2}$ connects the DC power supply to the circuit. The DC power supply is set to $10 \mathrm{~A}$ (which generates 10V) and must be manually ramped on and off to avoid the chance of sparks. The output of the circuit goes across the anti-Helmholtz coils which have a natural resistance $R_{\text {coil }}$ and a natural inductance $L_{\text {coil }}$. The circuit output, controlled by $S_{1}$ and $S_{2}$, is either a DC current of $10 \mathrm{~A}$ or $30 \mathrm{~Hz}$ pulsed current matching the signal at CONN1 from the pulse generator where the high output is $10 \mathrm{~A}$ and the low output is $0 \mathrm{~A}$.

When the switches are set to continuous mode, $S_{1}$ connects to ground and $S_{2}$ connects to a wire 

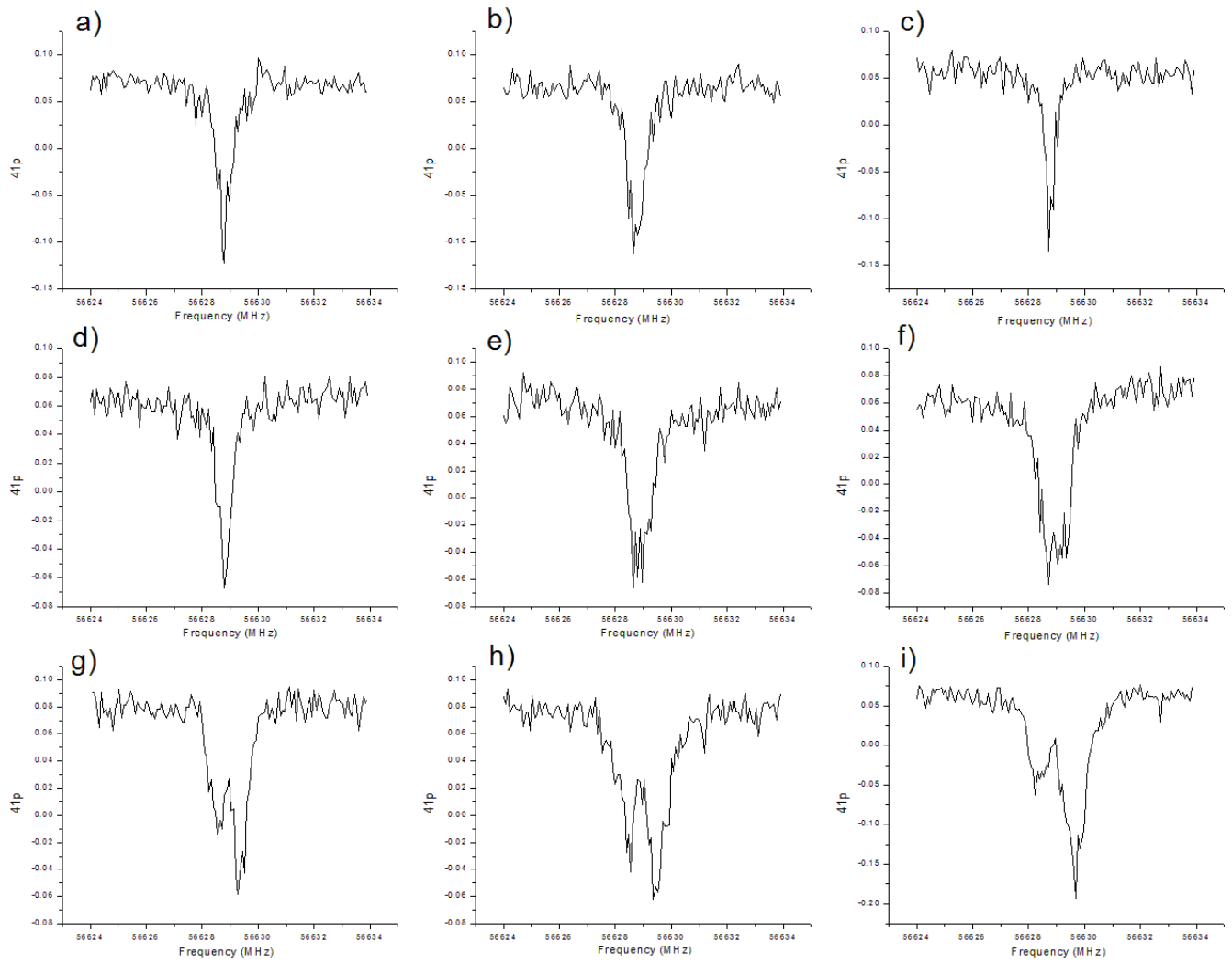

Figure 2.9: Shows the $41 s_{1 / 2} \rightarrow 41 p_{1 / 2}$ transition with a range of delay times a) $\left.8 \mathrm{~ms} \mathrm{~b}\right) 7 \mathrm{~ms} \mathrm{c}$ ) 6 ms d) $5 \mathrm{~ms} \mathrm{e)} 4 \mathrm{~ms} \mathrm{f)} 3 \mathrm{~ms} \mathrm{~g}$ ) $2 \mathrm{~ms} \mathrm{~h}) 1 \mathrm{~ms} \mathrm{i)} 0 \mathrm{~ms}$. The nulling coils were on and optimized before taking this data. 

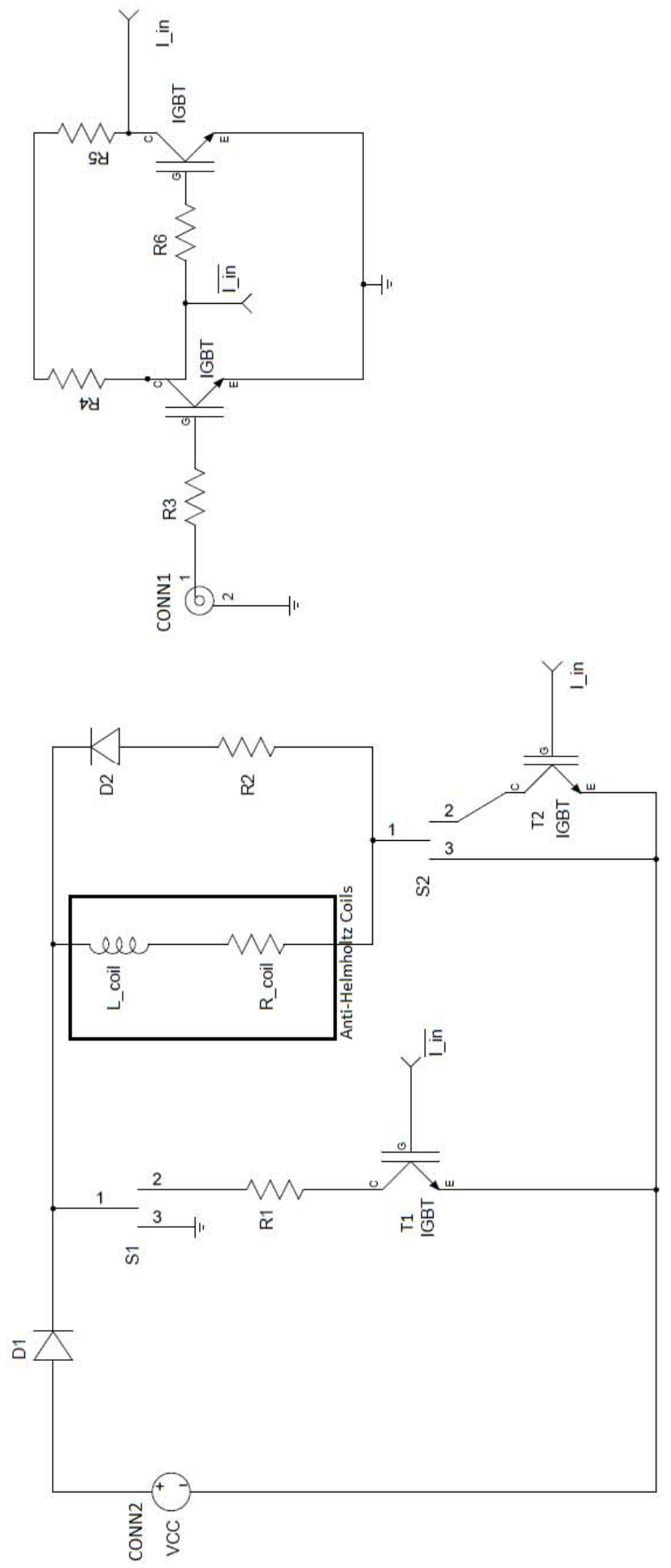

Figure 2.10: Circuit used to control magnetic field for MOT. Component values in Table 2.3. 


\begin{tabular}{|c|c|l|}
\hline Component & Value & Details \\
\hline$R_{1}$ & $1 \Omega$ & high power, air cooled \\
\hline$R_{2}$ & $20 \Omega$ & high power, air cooled \\
\hline$R_{3}$ & $10 \mathrm{k} \Omega$ & \\
\hline$R_{4}$ & $1 \mathrm{k} \Omega$ & \\
\hline$R_{5}$ & $1 \mathrm{k} \Omega$ & \\
\hline$R_{6}$ & $10 \mathrm{k} \Omega$ & \\
\hline$R_{\text {coil }}$ & $\sim 1 \Omega$ & Resistance of coil and vacuum chamber \\
\hline$L_{\text {coil }}$ & - & Inductance of coil and vacuum chamber \\
\hline$D_{1}$ & diode & \\
\hline$D_{2}$ & diode & \\
\hline$S_{1}$ & switch & connected to S2 \\
\hline$S_{2}$ & switch & connected to S1 \\
\hline$T_{1}$ & IGBT & Transistor \\
\hline$T_{2}$ & IGBT & Transistor \\
\hline$C O N N_{1}$ & $0-5 \mathrm{~V}$ & BNC connects to pulse generator \\
\hline$C O N N_{2}$ & $\sim 10 \mathrm{~V}$ & DC voltage supply-set by current at $10 \mathrm{~A}$ \\
\hline
\end{tabular}

Table 2.3: Values for components in the MOT Magnetic Field Switching circuit in Figure 2.10.

bypassing the transistor $T_{2}$. This allows the current to flow though the coils and not through any of the other resistors.

When the switches are set to switching mode, $S_{1}$ connects to $R_{1}$ and $T_{1}$, and $S_{2}$ connects to $T_{2}$. The transistors $T_{1}$ and $T_{2}$ are gated by $\overline{I_{i n}}$ and $I_{i n}$ respectively. $\overline{I_{i n}}$ and $I_{i n}$ are generated by the input pulse where ideally $\overline{I_{i n}}+I_{i n}=\mathrm{DC} 5 \mathrm{~V}$ signal. When one is high, the other is at ground. $I_{i n}$ is the same as the input pulse. When it is high, it shorts $T_{2}$ - this allows the current to pass through the coils creating the MOT magnetic field. When $I_{i n}$ is low and $\overline{I_{i n}}$ is high, current won't pass though the coils but through $R_{1} . R_{1}=1 \Omega$ is roughly the same as $R_{\text {coil }}$ so the power supply doesn't see a resistance jump. Since the coils have a natural inductance $L_{\text {coil }}$ it will dissipate though $R_{2}$. $D_{2}$ prevents current from flowing through $R_{2}$ for anything other than dissipating the induced current, and $D_{1}$ protects the power supply.

The present value of $R_{2}=20 \mathrm{Ohms}$ has not been carefully optimized. The way to do that would be do vary the value of $R_{2}$ using a potentiometer or multiple resistors and find the one that allows the shortest switching delay. Having the magnetic field off for a shorter time increases the amount of atoms for the blue laser to excite. Without the magnetic field the atoms are not trapped and can drift away from the beam path. 


\section{Nulling Coils}

Three sets of Helmholtz coils are used to null the external magnetic field. Setting the currents and therefore the fields produced by these coils is the premise of Section 2.5. The coils are on three orthogonal axes. Each pair of coils is independently controlled and used to counter the external magnetic field on one axis. These run at a much lower current than the MOT magnetic field coils and so do not need to be water cooled.

\subsubsection{Ultra High Vacuum Chamber}

An Ultra High Vacuum (UHV) is necessary to form the MOT. Vacuum pressures higher than $10^{-8}$ Torr do not allow the current setup to trap atoms [2]. To maintain the UHV a 2011s Physical Electronics ion pump is running at all times. If the ion pump turns off, it's necessary to turn it back on within 48 hours. Otherwise the pressure inside the chamber may rise above the upper limit of pressure of the ion pump $\left(10^{-4}\right.$ Torr). In that case (as well as whenever the chamber is opened), a roughing pump must be used to initially lower the pressure. Please see Park's thesis [2] for details on opening the pump and changing components.

The two components most likely to require servicing are the micro-channel plate detector (MCP) and Rubidium or Cesium getters. The MCP detector is used to detect the atoms as discussed in Section 2.2.2. The getters supply the atoms used in the MOT. We used Rubidium, but the chamber also has Cesium getters installed. When a current (supplied by a Kepco power supply) is sent though a getter, it "boils off" atoms. As the getter ages more current is needed to generate the same number of atoms in the chamber. There are multiple getters installed so if the one currently in use runs out, it's possible to merely change the external power source connections to start a new getter. This minimizes the need to open the chamber. When the chamber needs to be opened, it's recommended to change all getters and the MCP to minimize the number of times the chamber must be opened.

\section{$2.2 \quad$ Exciting and Detecting Rydberg Atoms}

Rydberg atoms are atoms in which a valance electron is excited to a high energy level. As discussed in the introduction, the have large and loosely bound orbits make Rydberg atoms very useful for studying interactions between atoms and external fields. One example of this is field ionization, 
described in Section 2.2.2, another is the dipole-dipole interaction. Studying them was the goal of the dressed state experiment described in Chapter 3. More information on Rydberg atoms can be found in Gallagher's Rydberg Atoms [1].

\subsubsection{Blue Laser}

\section{Overview}

The work described in this thesis was done with Rydberg atoms excited to $n=41$. The atoms were excited by a laser at $480 \mathrm{~nm}$ (see Table 2.1 for the exact wavelength). The laser must be tunable and have narrow linewidth so to excite only the desired state. Diode lasers are a good source of narrow linewidth, tunable laser light, however $480 \mathrm{~nm}$ diodes are not commercially available so a frequency doubled $960 \mathrm{~nm}$ diode laser is used. In addition, the light that reaches the MOT needs to be pulsed and of higher power than a diode laser traditionally emits, so several stages of amplification are necessary. The setup is pictured in Figure 2.11. Each stage and it's alignment procedure is outlined in the following subsections.

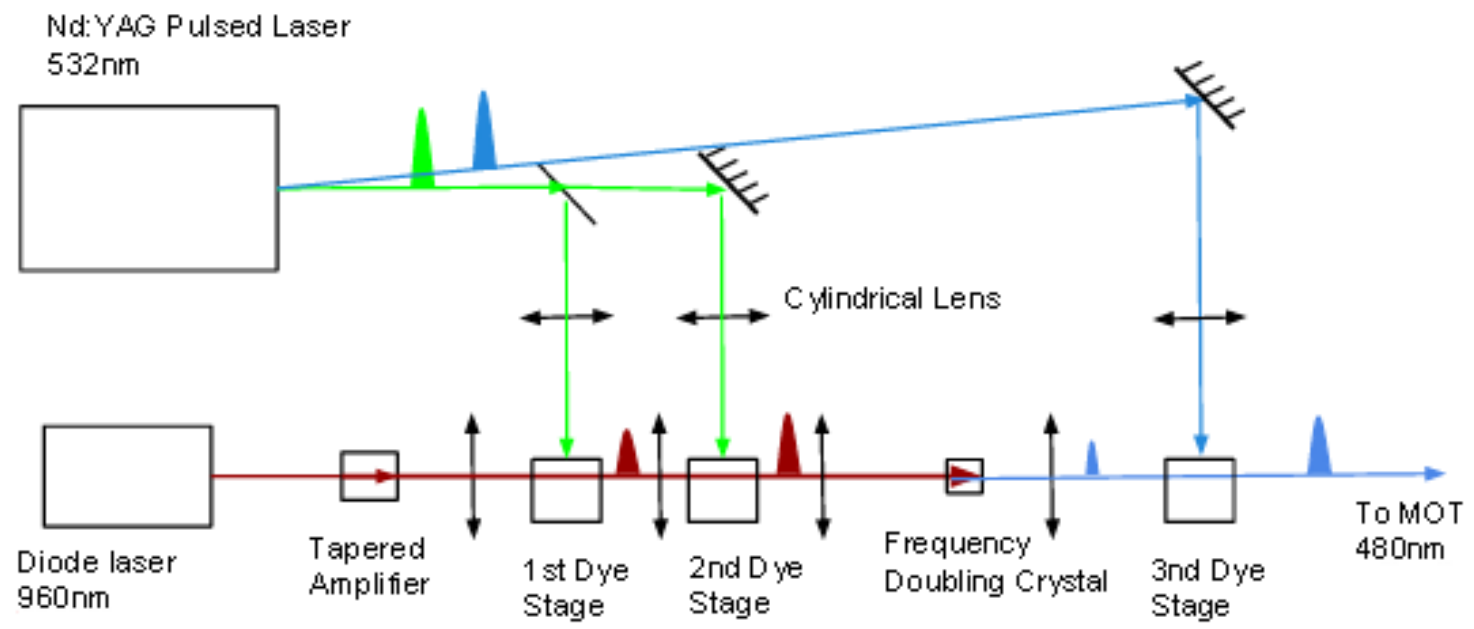

Figure 2.11: Diagram of how the $480 \mathrm{~nm}$ light used to excite the atoms to Rydberg states is created. 


\section{Toptica diode Laser}

The initial light comes from a Toptica Photonics diode laser (DL100). See Table 2.2 for typical current and temperatures. The laser is a Littrow external cavity diode laser tuned through the diffraction grating position, current, and temperature to get the desired wavelength. Fine tuning is done by changing the $\mathrm{DC}$ voltage sent to the piezo controlling the cavity length. It is important for there not to be mode hops within $\sim 0.003 \mathrm{~nm}$ or for $\sim 80 \%$ of the piezo range of either side of the resonance. This laser is generally not locked, but will drift off resonance over the course of an hour (more or less depending on the day). If checked and retuned regularly, this is not a problem.

The laser beam is sent to a Fabry-Perot cavity (Coherent spectrum analyzer) to check that it is single mode. The picked off light from the first isolator after the tapered amplifier is guided into the Fabry-Perot cavity. The cavity length is ramped over the laser wavelength and it outputs a frequency spectrum with peaks at the laser frequencies. The laser is single mode if only one peak can be seen in the spectrum over the length of one cavity piezo cycle. It is possible to use this peak to lock the laser. However, the length of the cavity drifts with time and temperature shifts. Locking the laser to the cavity actually decreased the amount of time the laser was on resonance. A way to solve this problem is to first lock the cavity to different laser that is locked with a saturated absorption or some other method, and then lock the $960 \mathrm{~nm}$ laser to the locked cavity. However, for the purposes of this thesis, locking was not necessary.

\section{Tapered Amplifier}

A tapered amplifier (TA) is essentially a diode laser without end mirrors. It can produce much higher output power replicating the seed beam in all characteristics - including mode, polarization, and frequency. The light from the $960 \mathrm{~nm}$ diode laser is around $60 \mathrm{~mW}$. When properly aligned through the tapered amplifier, it is possible to get more than $400 \mathrm{~mW}$ of power. Proper alignment is delicate and requires mode-matching. The TA emits a low power guide beam in the direction of the input, and a amplified beam from the output. This guide beam needs to be matched in direction and size with the input beam. Figure 2.12 shows the set up used for proper alignment. Mirrors 1 and 2 are used to match the guide beam in translational space. Lens 2 is placed on a translation stage, and by shifting it on the axis parallel to the beam path it's possible to approximately match the guide beam in size. 


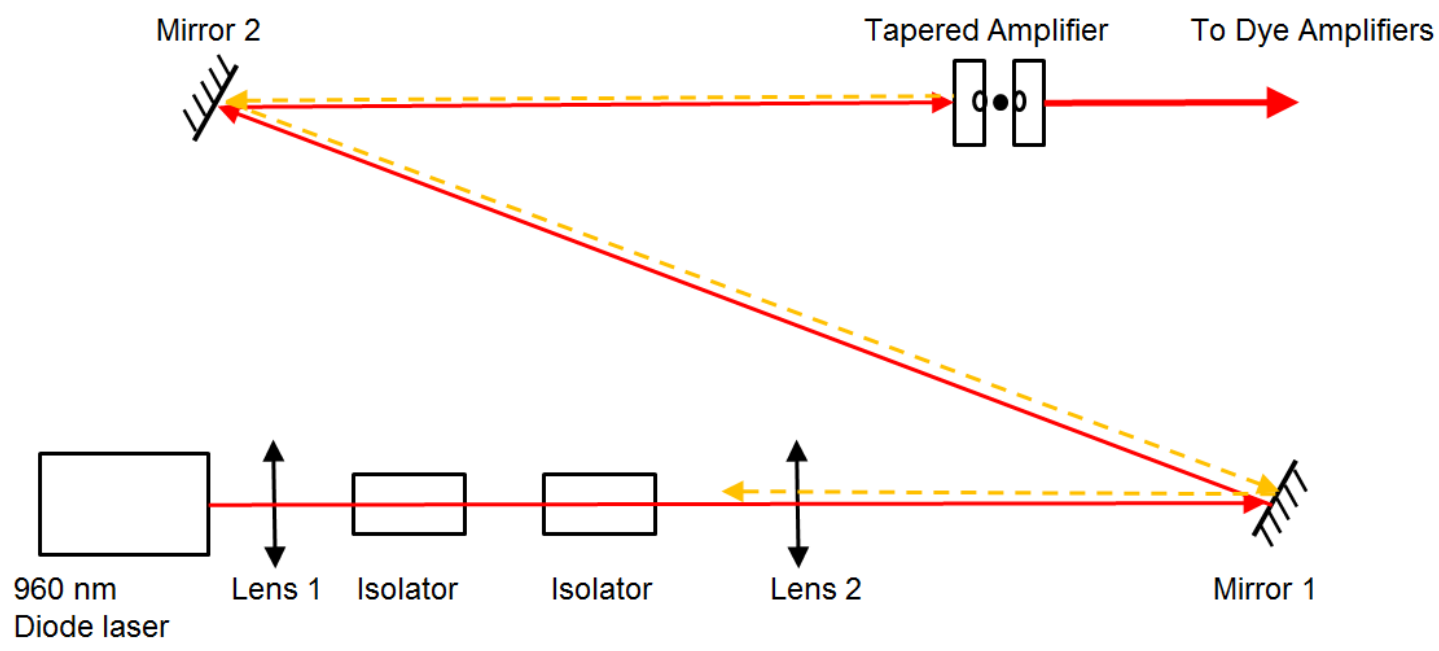

Figure 2.12: Diagram of tapered amplifier's components necessary for alignment. The red solid line is the beam from the diode laser. The orange dashed line is the guide beam.

\section{Dye Amplification Stages and Frequency Doubling Crystal}

We use two dye amplification stages to pulse amplify the continuous $960 \mathrm{~nm}$ diode laser. The dye is pumped through glass cells and excited using the second harmonic of a Nd:YAG Spectra-Physics Quanta-Ray laser. The laser is focused to a line just within the front of the dye cell. This is the pump beam. The $960 \mathrm{~nm}$ beam from the diode laser enters the cell from the side along the excitation line of the pump beam. It pulls out the energy of the excited molecules in the dye into a pulse. This pulse is then amplified in a second, identical stage. Next, the light passes through a Potassium Niobate $\left(\mathrm{KNbO}_{3}\right)$ crystal. Through second harmonic generation in the crystal, $480 \mathrm{~nm}$ light is produced. Optionally the laser beam can go through a third dye stage excited by the YAG laser third harmonic that adds power and is useful for experiments that require a high density of Rydberg atoms. Figure 2.11 shows the dye stages and frequency doubling crystals.

To create a dye amplification stage one needs:

- a pump laser

- the laser to be amplified

- a cylindrical lens (ideally on a translation stage) 
- mirrors (totally or partially reflective as necessary for multiple stages) to control the pump and $960 \mathrm{~nm}$ beams

- 2 spherical lenses

- a dye cell

- a motor to pump the dye

- 0.5-1 L container of the appropriate dye (See Table 2.4)

- tubing to connect the dye cell, motor, and dye container.

The dye tubing and the parts to which it is attached can be secured with zip ties. It is advisable to run methanol or something similarly benign through a new dye stage initially to both clean it out and to check for leaks. Once it has run for 30 minutes without leaks, it should be emptied and the dye reservoir attached. The dye cell needs to be placed such that the beam to be amplified (the $960 \mathrm{~nm}$ beam) runs parallel to the face of the cell and as close to the glass face as possible while remaining in the dye. The two spherical lenses can be placed with one before the cell to decrease the size of the beam in the dye and one after to re-collimate it. Resizing the laser can increase the amount of power it can pull from the excited dye.

After the beam to be amplified is aligned, the pump beam (one of the YAG harmonics) is sent to hit the front of the dye cell. The cylindrical lens is used to focus the pump beam into a line. The cylindrical lens should be placed at a distance from the cell such that it focuses within the dye, and the vertical width should match the width of beam to be amplified. Through manipulating the cylindrical lens rotation and the final pump mirror, it is possible to align the pump beam and the beam to be amplified such that they will pass through the same section of dye. The pump beam will excite the dye molecules in a pulse, then the beam being amplified will pull that power out producing a high power pulse of $960 \mathrm{~nm}$ light. The pump motor circulates fresh dye to be excited. The dye will need to be replaced when the output beam slowly loses power over the course of a couple days. The larger the dye reservoir, the longer the dye will last. For example, $0.5 \mathrm{~L}$ of dye across 2 stages with use every day lasts about 2 months. Using $1 \mathrm{~L}$ of dye instead should double that lifetime. For maximum power output, both the pump and the probe beams should be vertically polarized so their polarizations match. This is a larger problem for some dyes and doesn't seem to be crucial for the 
dyes used in this thesis. The first two stages have their polarizations naturally aligned. In the third stage, the gain from changing the polarization was approximately canceled by the power lost going through Fresnel Rhombs used to change the polarization. The dye mixtures used are given in Table 2.4 .

\begin{tabular}{|l|l|l|l|l|}
\hline Frequency & Dye & Pumped with & g/L dye & solvents \\
\hline $960 \mathrm{~nm}$ & LDS965 & YAG 532 nm & 0.12 & .85 propylene carbonate \\
\cline { 4 - 5 } & & & .15 ethylene glycol \\
\hline $480 \mathrm{~nm}$ & Coumarin 480 & YAG 355 nm & 0.4 & 1.00 methanol \\
\hline
\end{tabular}

Table 2.4: Dyes used for dye stages

The frequency doubling crystal is on a platform that controls tilt on two axes to optimize the phase matching. More detail on frequency doubling can be found in Appendix A of Wenhui Li's thesis [7]. After the crystal is a prism which separates the $960 \mathrm{~nm}$ and $480 \mathrm{~nm}$ beams. After the prism, the $960 \mathrm{~nm}$ light is ignored, and the 480nm light is used for alignment. Since $480 \mathrm{~nm}$ can be seen with the naked eye, it's easiest to optimize visually both crystal alignment and the dye stages. Without dye amplification, the best possible $480 \mathrm{~nm}$ light output from the crystal is on the lower limit of what can be seen. However, any dye amplification will greatly increase the blue light visibility. One trick for fine tuning adjustments is to deliberately attenuating the light so that smaller changes in power are easily seen.

\section{Triggering the Nd:YAG laser}

All the pulse generators with their various delays are triggered from the YAG Q-switch sync output. The YAG has two triggers: the lamp trigger turns on the lamps to excite the Nd:YAG rod, and the Q-switch trigger controls the Pockels cell that allows the laser light to escape in a pulse once it has reached maximum gain. For this reason, it needs to be delayed from the lamp switch trigger. The delay (generally between $150 \mu s$ and $200 \mu s$ ) that optimizes the power output is based on how quickly the laser rod reaches maximum stored energy and is slightly different for each laser. There are three alternatives for triggering the YAG laser, each with different benefits.

1. Use internal triggers. This is the simplest option, however the internal YAG trigger phase shifts relative to the $\mathrm{AC}$ line changing the $\mathrm{AC}$ pick up on the signal line. This creates a background shifting that distorts the transition lineshapes as seen in Figure 2.13 a. This can generally be 


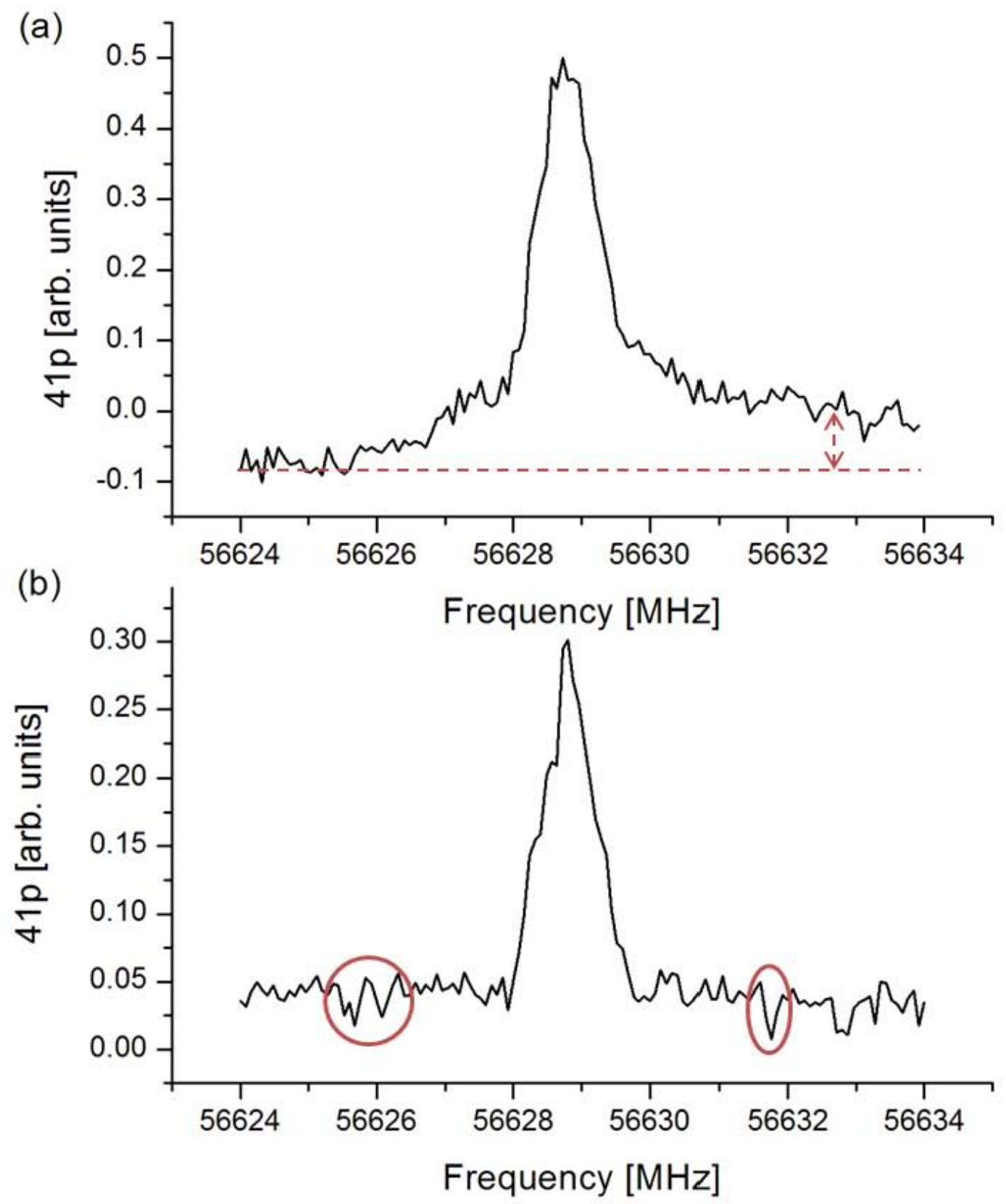

Figure 2.13: Graph a) shows a scan triggered off the internal trigger compared to graph b) triggered off the AC line. Note with graph a) there is a change in the background level from low frequency to high frequency. With graph b) there are sharp drops which are small in the background, but render a scan useless if they occur on the peak. 
countered by averaging at least 8 data runs.

2. Sync the lamp trigger to the $\mathrm{AC}$ line/2 $(30 \mathrm{~Hz})$. This fixes the background shifting problem but introduces another one - due to the way the triggering circuit is designed, the laser will skip laser shots when the circuit comparator doesn't trigger off the AC line. This causes the trigger output to miss a cycle or half cycle. At times the trigger skips frequently, and it isn't possible to take reliable data. For several months the circuit skipped less than twice a day-easy to work around. Then over the next summer it skipped a shot every couple seconds for several weeks. It should be possible for this flaw in the circuit to be fixed. The synchronizing circuit is described below and pictured in Figure 2.14.

3. Sync the Q-switch to the $\mathrm{AC}$ line/4 $(15 \mathrm{~Hz})$ and the lamp trigger to the $\mathrm{AC}$ line $/ 2(30 \mathrm{~Hz})$. The YAG lamps need to be triggered at $30 \mathrm{~Hz}$ and will not trigger at a different rate without reconfiguration of lensing and disabling a interlock circuit by a Spectraphysics technician. However, it is possible to trigger the Q-switch at $15 \mathrm{~Hz}$ (yielding laser output at $15 \mathrm{~Hz}$ ). This is useful when working with high densities of Rydberg atoms where a larger percentage of the MOT is excited to Rydberg levels. Triggering at $15 \mathrm{~Hz}$ instead of $30 \mathrm{~Hz}$ allows the MOT twice the time to rebuild its population, resulting in more trapped atoms to be excited. However, triggering is from the $\mathrm{AC}$ line and suffers the same problems as option 2 and requires an extra SRS Delay/ Pulse Generator.

YAG Triggering Circuit The YAG triggering circuit is diagrammed in Figure 2.14. Starting from the $\mathrm{AC}$ line, the signal goes through a diode which outputs only the top half of the sine wave and then a voltage divider to bring it into the range of $5 \mathrm{~V}$. Next it goes through a comparator, LM311, which turns the half-sine wave into a square wave. $P O T_{1}$ creates a variable voltage divider that determines where on the curve the square wave starts. If turned to ground, it remains a flat line at ground. If tuned too far in the other direction it triggers at the top of the half-sine and the square wave output rails high. Triggering in between the two extremes decreases the likelihood of the comparator missing the AC line signal and the circuit not triggering.

Next the $60 \mathrm{~Hz}$ square wave goes to a flip-flop circuit set up as a frequency divider. This outputs a $30 \mathrm{~Hz}$ square wave to $\mathrm{BNC} C O N N_{1}$ which can be used to trigger the laser at $30 \mathrm{~Hz}$. The trigger can be inputted to the YAG lamp trigger on the power supply and the trigger switch on the YAG 


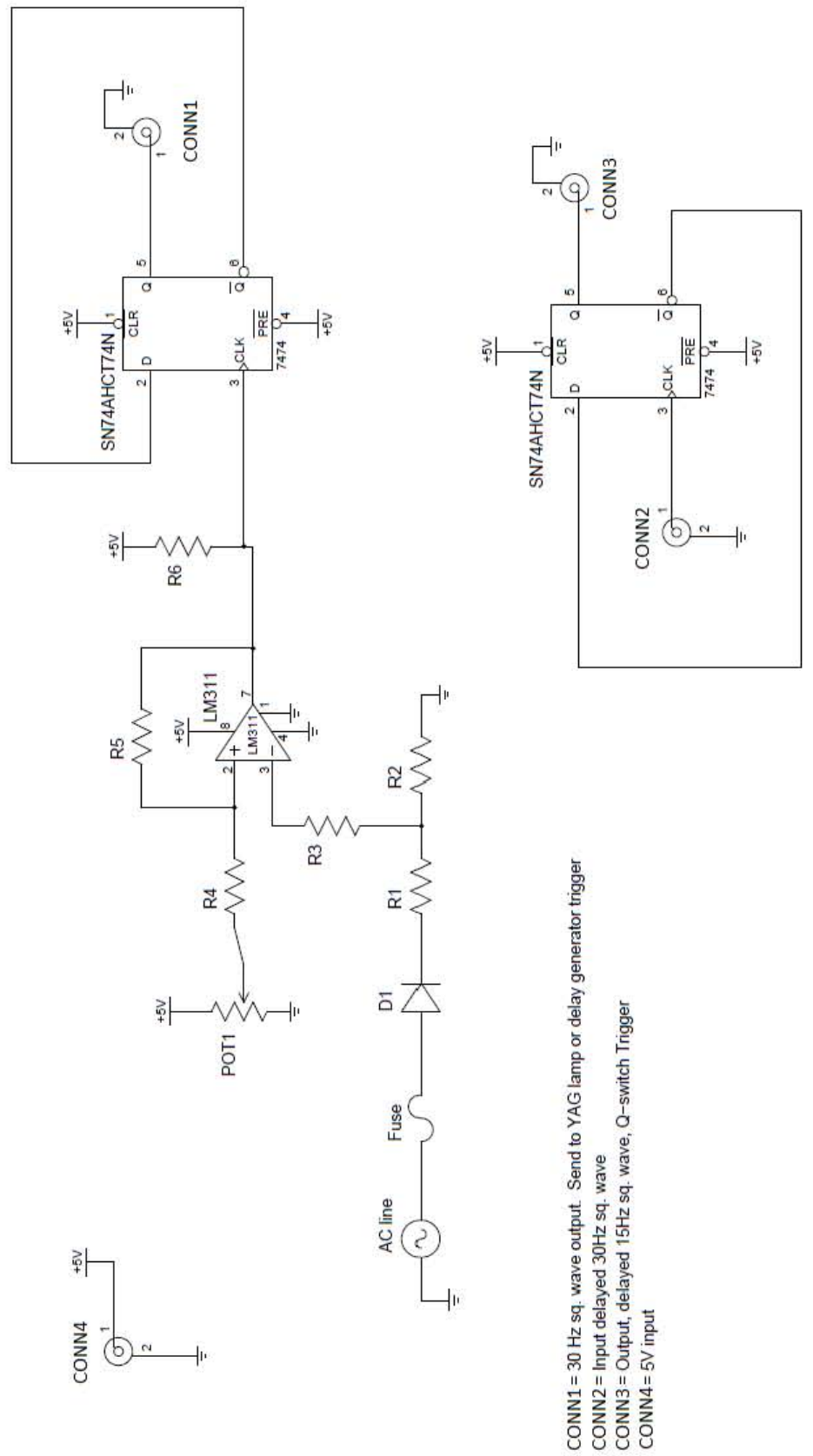

Figure 2.14: Circuit used to trigger the YAG laser at either $15 \mathrm{~Hz}$ or $30 \mathrm{~Hz}$ - see Table 2.5 for component values. 


\begin{tabular}{|l|l|l|}
\hline Component & Value & Details \\
\hline R1 & $1.5 \mathrm{M} \Omega$ & \\
\hline R2 & $50 \mathrm{k} \Omega$ & \\
\hline R3 & $1 \mathrm{M} \Omega$ & \\
\hline R4 & $10 \mathrm{k} \Omega$ & \\
\hline R5 & $1 \mathrm{M} \Omega$ & \\
\hline R6 & $1 \mathrm{k} \Omega$ & fast blow \\
\hline POT1 & $2 \mathrm{k} \Omega$ & Half-sine output \\
\hline Fuse & $1 \mathrm{~mA}$ & Turns half-sine into $60 \mathrm{~Hz}$ sq wave \\
\hline D1 & $V_{\text {rev,rms }}=100 V, 1 m A$ & set up as frequency divider \\
\hline LM311 & Comparator & set up as frequency divider \\
\hline Flip-flop 1 & SN74AHCT74N & $\begin{array}{l}30 \text { Hz sq. wave output. } \\
\text { Send to YAG lamp trigger for } 30 \mathrm{~Hz} \\
\text { trig.newline or delay generator for } 15 \mathrm{~Hz}\end{array}$ \\
\hline Flip-flop 2 & SN74AHCT74N & Input delayed 30 Hz sq. wave \\
\hline CONN1 & BNC & $\begin{array}{l}\text { Output delayed } 15 \text { Hz delayed sq. wave } \\
\text { Q-switch trigger }\end{array}$ \\
\hline CONN2 & BNC & 5V input \\
\hline CONN3 & BNC & \\
\hline CONN4 & BNC &
\end{tabular}

Table 2.5: Values for components in the YAG Triggering circuit in Figure 2.14.

controller set to EXT. With triggering from the AC line in this fashion there is little power change from shot to shot, unless the trigger misses completely which results in no power for that shot. Since all other pulses trigger off the Q-Switch output of the laser, everything skips a pulse, and the MOT is generally diminished for several shots if it is in the switching mode (see section 2.1.4).

There are times when it is useful to trigger the YAG at $15 \mathrm{~Hz}$ described in option 3. To do so it is necessary to take the $30 \mathrm{~Hz}$ trigger from $\mathrm{BNC} C O N N_{1}$ in the circuit in Figure 2.14 and send it to a delay generator. Then, a $30 \mathrm{~Hz}$ pulse can be sent to the laser lamp trigger from one of it's outputs with no or minimal delay. A second pulse, delayed the appropriate amount of time to optimize the laser, is sent back to the triggering circuit into $\mathrm{BNC} C O N N_{2}$. It goes through another flip-flop circuit that divides it into a $15 \mathrm{~Hz}$ pulse. This outputs through BNC CONN 3 and should then be sent to the YAG Q-switch trigger. On the laser controller, the lamp triggering and Q-switching triggering switch need to be changed to external. If everything is set up, it is a simple matter of changing the Q-switch switch from Q-SW (internal) to EXT or back to allow the laser to be changed from $15 \mathrm{~Hz}$ to $30 \mathrm{~Hz}$. Since all other pulses are triggered from the Q-switch sync output, most of the triggering for other devices and circuitry can remain unchanged. The only part that 
requires adjustment is the magnetic field switching pulse. It needs to be set such that the field is on for all but $6 \mathrm{~ms}$ per cycle (see Section 2.1.4). If it is forgotten the MOT flickers or doesn't form, as the magnetic switching coils are on for 1 cycle but not the next. It is a good idea to compare the magnetic coils switching trigger pulse to the Q-switch pulse to make certain that it is as desired.

\subsubsection{Detecting the Rydberg Signal}

We detect our atoms using Field Ionization (FI) and the resulting ions or electrons are sent to a micro-channel plate detector (MCP). Rydberg atoms are in large loosely bound orbits, so it's experimentally possible to apply a large external electric field that will ionize the atoms [1]. Figure 2.15a demonstrates how the applied electric field tips the potential energy well of the atom, allowing higher level electrons to escape. The field is a pulse which rises almost linearly until it's maximum in approximately $2 \mu \mathrm{s}$. The electrons from different energy levels are ionized in order of increasing binding energy (See Figure 2.15b). Since the ions or electrons will reach the MCP at different times, it is possible to separate and identify the signal from the atoms in different Rydberg states. The $\mathrm{MCP}$ has to be calibrated so that it is possible to know the approximate number of atoms in each Rydberg state (see Section 2.4.2).

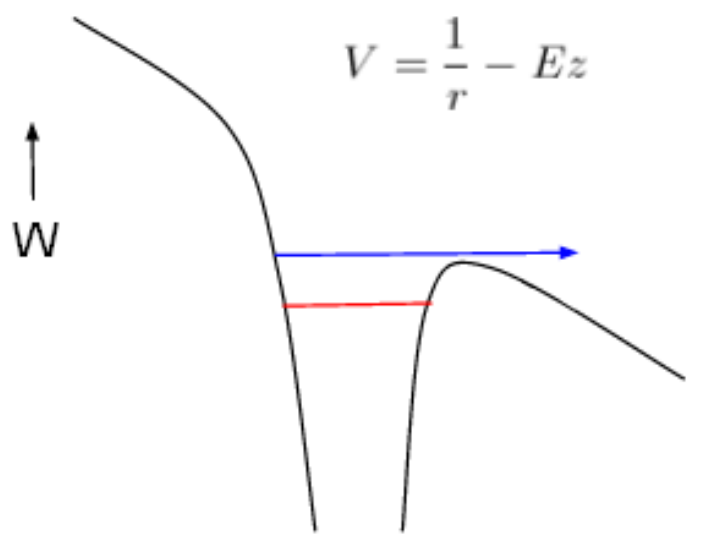

(a) An electric field applied to the atoms tips the potential well such that loosely bound electrons are able to escape.

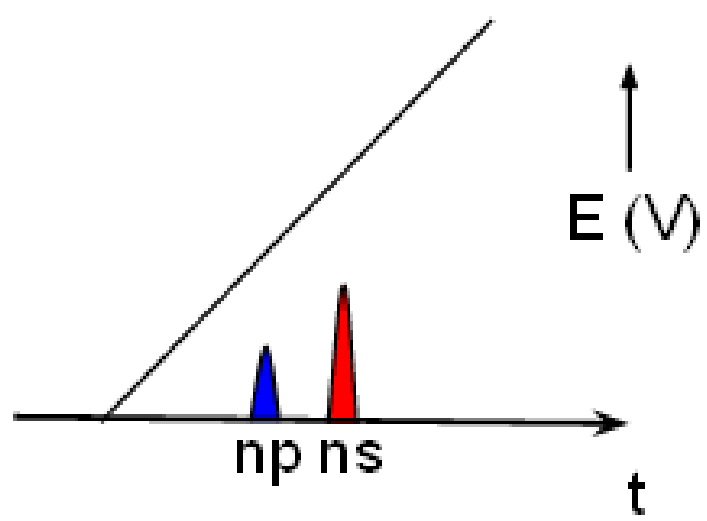

(b) Timing diagram for Field Ionization pulse.

Figure 2.15: Field Ionization Pulse Diagrams.

Once the atoms have been ionized, either the ions or electrons are detected by a MCP. The signal 
(a)

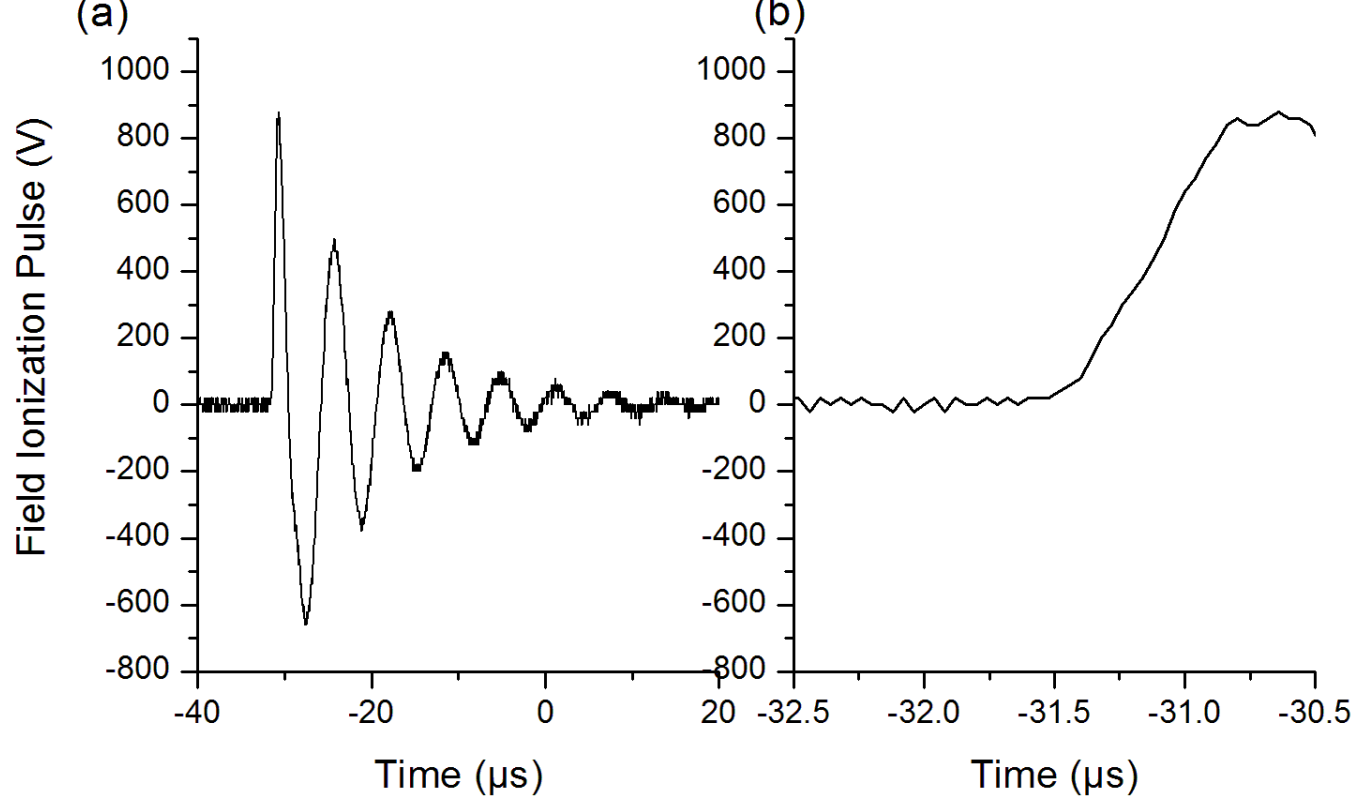

Figure 2.16: Graph of a field ionization pulse a) full pulse, b) The part of the pulse actually used to ionize the atoms. By fitting the linear slope, the rising time for the peak is approximated to $1.3 \mathrm{kV} / \mu \mathrm{s}$. 
is sent to a SRS gated integrator where the desired signal is gated, and the integrated signal is sent to the computer through a National Instruments BNC-2110 or BNC-2115. A Labview program then collects these values while scanning a microwave synthesizer over a range of frequencies.

The FI pulse is generated using the circuit shown in Figure 2.17. The voltage pulse output is sent to two of the four rods surrounding the MOT to create the field. The rods are $0.165 \mathrm{~cm}$ in diameter and positioned at the corners of a square with sides length $1.78 \mathrm{~cm}$ [7]. Figure 2.16 shows the image of a FI pulse sent to the rods by the circuit.

Field Ionization Circuit Describing the circuit components: $C O N N_{1}$ is connected to a DC voltage set within the range of 30-100V (generated by a Sorensen DC power supply) which charges the capacitor $C_{1}$. $R_{1}$ protects the power supply as well as determining the $\mathrm{RC}$ constant and therefore the time necessary to charge the capacitor. That time needs to be less than the time in between FI pulses (triggered from the YAG laser shot). When a trigger pulse is sent from a BNC Pulse Generator to $\mathrm{CONN}_{2}$ and the gate of the SCR6405 chip, the chip becomes a diode connecting the transformer to ground and effectively shorting the capacitor causing it to discharge across the transformer. The switch serves to change the direction of the current flow and therefore the sign of the output pulse. The two zener diodes (Model \#1N4746) slow the pulse's rising time. When the charge stored in the capacitor goes across the transformer a corresponding, amplified pulse is outputted to $\mathrm{CONN}_{3}$ and is sent to the rods in the vacuum chamber that create the field. Since this pulse rises sharply at a rate on the order of $1 \mathrm{kV} / \mu s$, there is ringing afterwards. A resistor $R_{3}$ can be used to damp this ringing. However, $R_{3}$ also damps the overall amplitude of the pulse. Figure 2.18 shows the FI pulse with a)no resistance across the output $\left(R_{3}=\infty\right)$, b) $R_{3}=100 k \Omega$ c) $R_{3}=22 k \Omega$ and d) $R_{3}=4.4 k \Omega$, all with $\mathrm{DC}$ voltage $=30 \mathrm{~V}$. For all data taken before May 23, 2012, $R_{3}$ was not in place. Afterwards $R_{3}=100 k \Omega$ was used.

In these experiment, unless stated otherwise, the MCP and FI pulse are set up to detect electrons. However, it is possible to switch between detecting ions and electrons, by changing three things:

1. MCP voltage supplied, positive for electrons, negative for ions

2. MCP circuit switched as described in the circuit diagram in Figure 2.19

3. FI pulse circuit switch flipped (see Figure 2.17) 


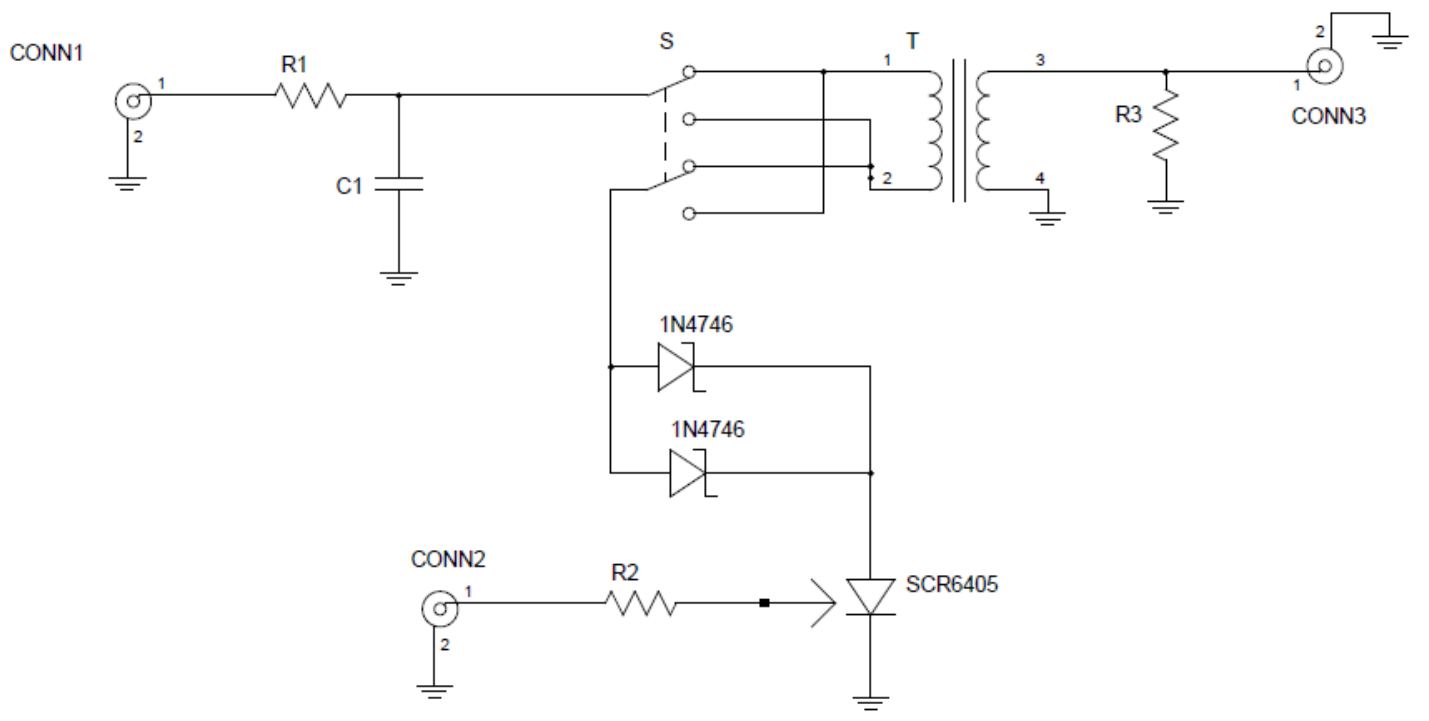

Figure 2.17: Circuit used to generate Field Ionization Pulse (FI)- see Table 2.6 for component values.

\begin{tabular}{|l|l|l|}
\hline Component & Value & Details \\
\hline R1 & $8 \Omega$ & \\
\hline R2 & $51.8 \Omega$ & \\
\hline R3 & $100 \mathrm{k} \Omega$ & Optional \\
\hline C1 & $47 \mu F$ & \\
\hline S & Switch & to detect ions or electrons \\
\hline D1 & $1 \mathrm{~N} 4746$ & \\
\hline D2 & 1N4746 & \\
\hline T & Trigger Transformer, T228 & Gain 30x \\
\hline SCR6405 & SCR6405 & gate \\
\hline CONN1 & BNC & DC Voltage, 30-50V \\
\hline CONN2 & BNC & Trigger pulse, $5 \mathrm{~V}$ \\
\hline CONN3 & BNC & FI pulse output \\
\hline
\end{tabular}

Table 2.6: Values for components in the Field Ionization circuit in Figure 2.17.

\begin{tabular}{|l|l|}
\hline Component & Value \\
\hline R1 & $100 k \Omega$ \\
\hline R2 & $11.3 M \Omega$ \\
\hline R3 & $15 \Omega$ \\
\hline C1 & unknown \\
\hline
\end{tabular}

Table 2.7: Values for components in the MCP circuit in Figure 2.19. 

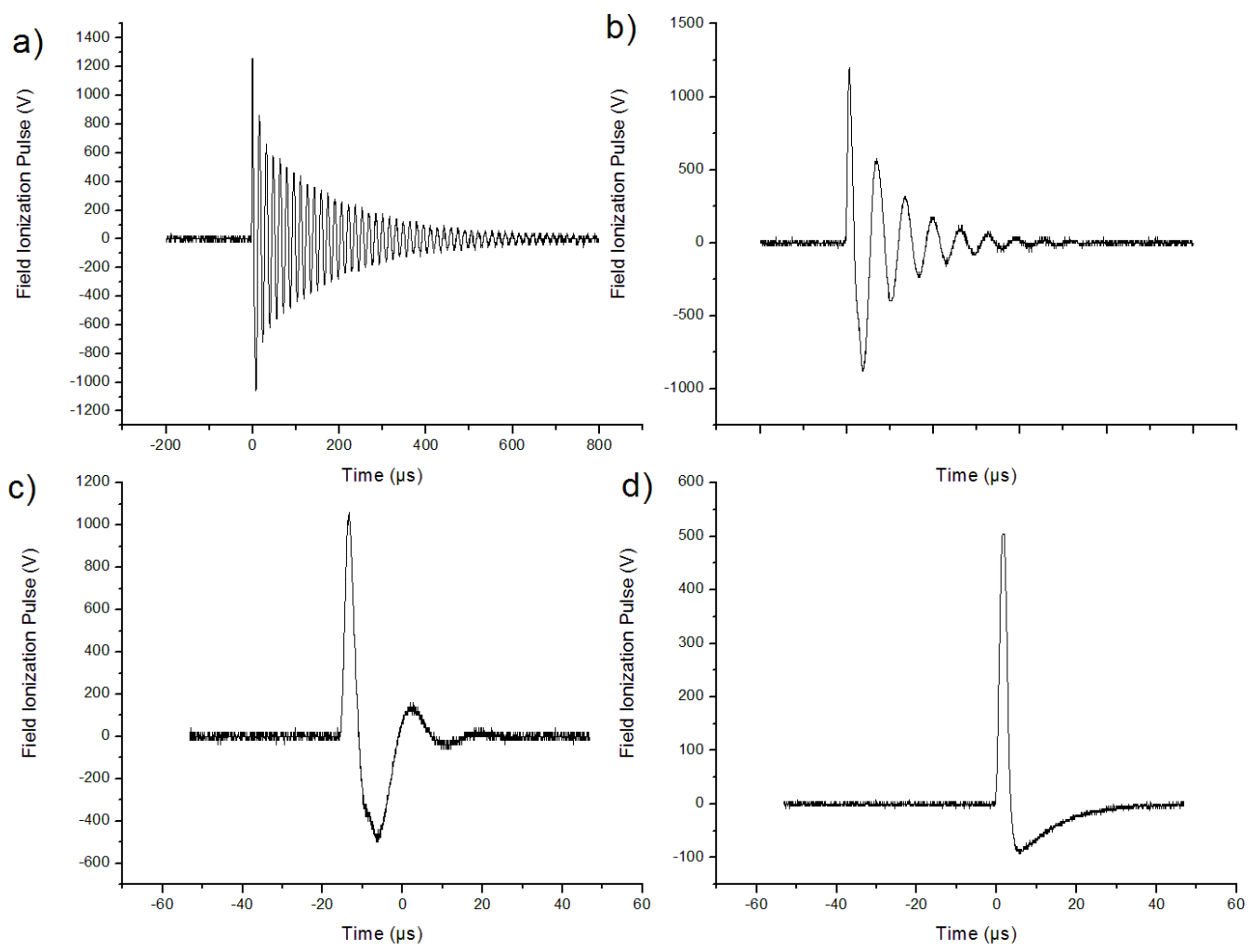

Figure 2.18: FI pulse with a)no resistance across the output $\left(R_{3}=\infty\right)$ b) $R_{3}=100 k \Omega$ c) $R_{3}=22 k \Omega$ and d) $R_{3}=4.4 k \Omega$ 


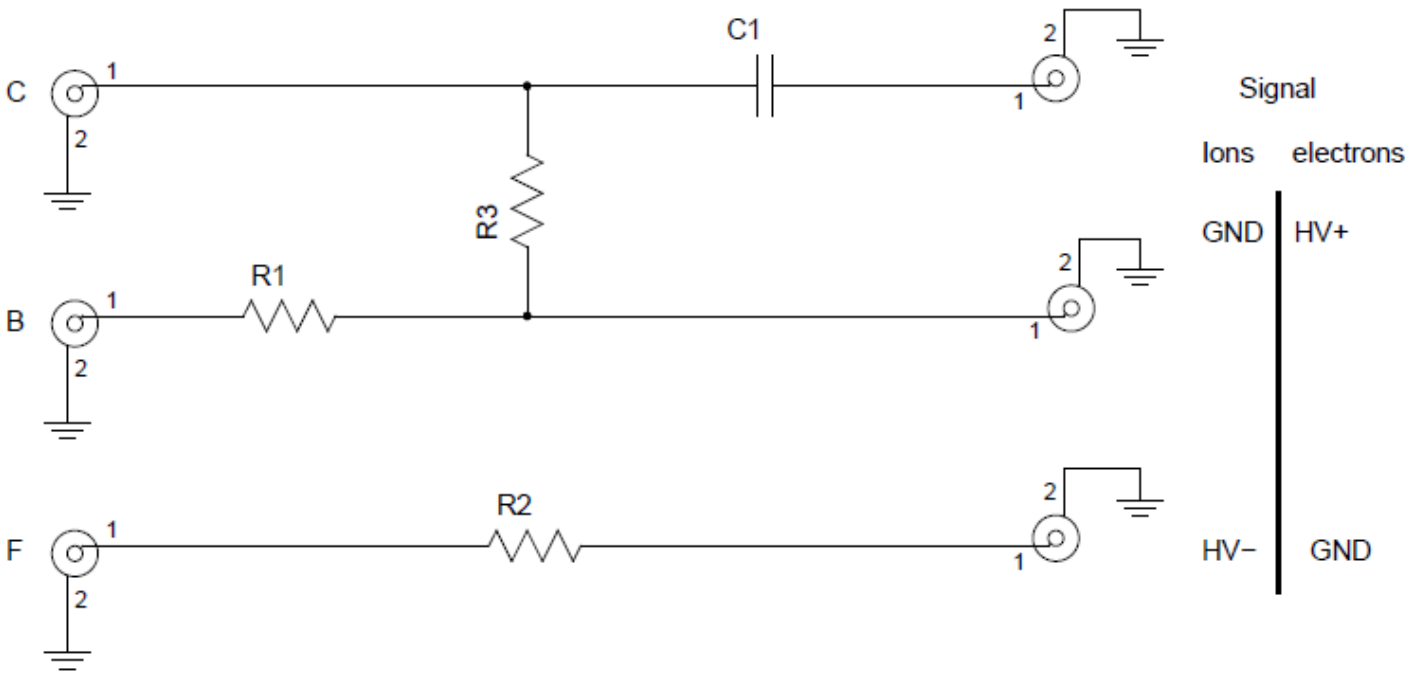

Figure 2.19: Circuit used to change MCP between detecting ions and electrons-see Table 2.7 for component values.

\subsection{Microwave Spectroscopy}

Once the atoms have been excited to a Rydberg level they can be changed to different states through the use of microwave pulses. See Table 2.1 for the transitions and frequencies used in this thesis. By scanning over the resonance of one of these transitions, it is possible to learn about the state based on the lineshape. The resonance frequencies correspond to energy differences between energy eigenvalues. They can be broadened both symmetrically and asymmetrically. Common causes of broadening that can be controlled are stray fields (see Sections 2.1.4 and 2.5), microwave probe power, microwave pulse length, and Rydberg atom density (tied to dipole broadening). In Figure 2.21 there is a transform limited resonance lineshape, which is the narrowest lineshape possible for a given microwave pulse length. More detail about getting a transform limited pulse can be found in Section 2.5.

The microwave fields are generated by a microwave synthesizer (HP 83620 synthesized sweeper), pulsed, and pass through frequency multipliers and variable attenuators to be broadcast from a microwave horn into the vacuum chamber of the MOT. Figure 2.20 shows the specific setup for the two microwave fields used in this thesis. The frequency of these pulses can be controlled through 


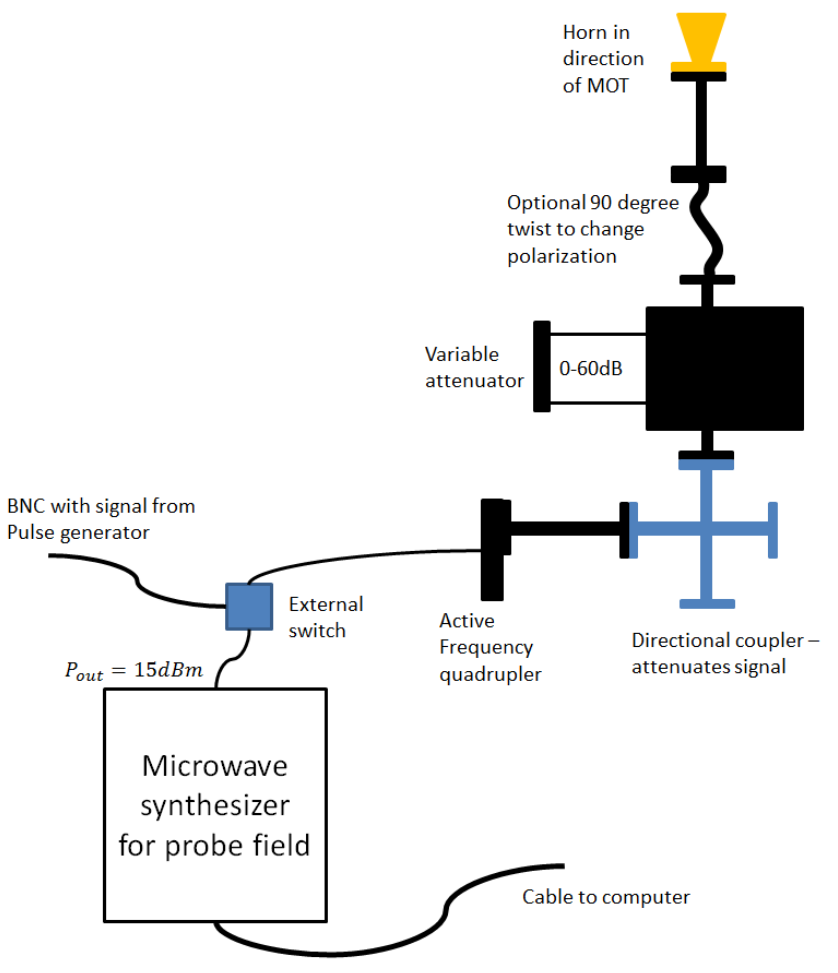

(a) Probe microwave field setup

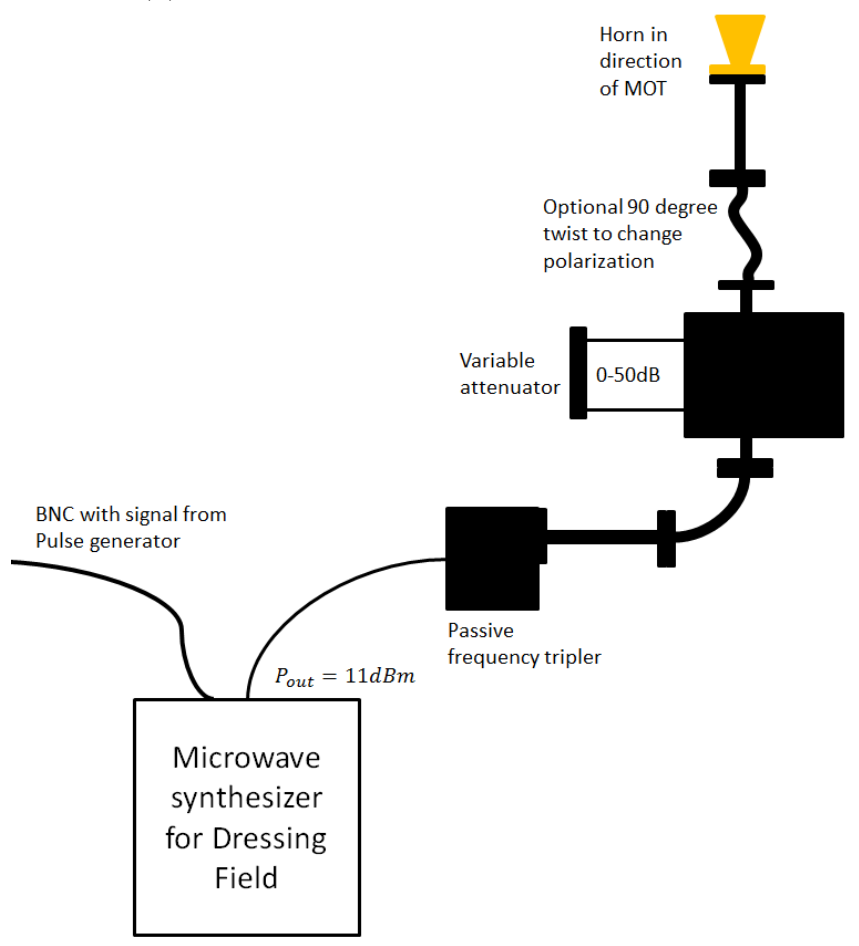

(b) Dressing microwave field setup

Figure 2.20: Setups for the two microwave fields used in this thesis. 
either the synthesizer interface (dressing field) or a Labview program (probe field). The pulse timing and length comes from a delay pulse generator (SRS Model DG535 4 channel digital delay /pulse generator) triggered from the YAG laser Q-switch external trigger. This is sent to an external switch for the probe microwave field. The dressing field used in Chapter 3 comes from a second microwave synthesizer where the trigger pulse is sent directly to the synthesizer and it's output is pulsed.

The frequencies required for the atomic transitions used are in the 50-60 GHz range. As the frequency synthesizers generate fields in the $10 \mathrm{MHz}-20 \mathrm{GHz}$ range, they are sent through either a tripler, a quadrupler, or two doublers to get to the appropriate frequency. Power levels of the microwave fields are controlled through the synthesizer power out $\left(P_{\text {out }}\right)$ and then attenuated by variable attenuators after the frequency multiplying elements. This is essential as additional power can cause broadening. The components after the frequency multipliers are connected by waveguide up to where they are emitted through a microwave horn. The emitted polarization can be changed through the use of $90^{\circ}$ twist waveguides. The active quadupler and doublers require $P_{\text {out }}$ of the synthesizer of $\geq 15 \mathrm{dBm}$. As a result they require a fixed attenuation (of more than 50dB) and a variable attenuator $(0-50 \mathrm{~dB})$ to bring the microwave pulse power down into the range where there is no power broadening. If a large enough fixed attenuator can't be found, then a directional coupler can be used to attenuate the signal. The passive tripler (HP 83556A mm-wave source module) can be run at lower power and only needs 1 variable attenuator on its output.

\subsection{System Parameters}

\subsubsection{The Number of Atoms in the MOT and in the Rydberg state.}

Knowing the number of atoms is the first step in calculating the average density of the MOT and of the Rydberg atoms. The density determines the strength of the inter-atomic interactions. The number of atoms can be calculated using the florescence of the MOT described by the method below.

A power meter is placed at the focus of a lens outside the vacuum chamber. For this set up, the solid angle, $\Delta \Omega$ can be calculated (equation 2.3) and used to convert the power from the MOT

florescence detected by the power meter $\Delta P_{M O T}$ to the total power emitted by the MOT $P_{M O T}$ in equation 2.4. $\Delta P_{M O T}$ is the change in power detected by the power meter relative to the background (power with MOT minus power without MOT). Equation 2.5 can be used to calculate the energy $E_{f}$ 


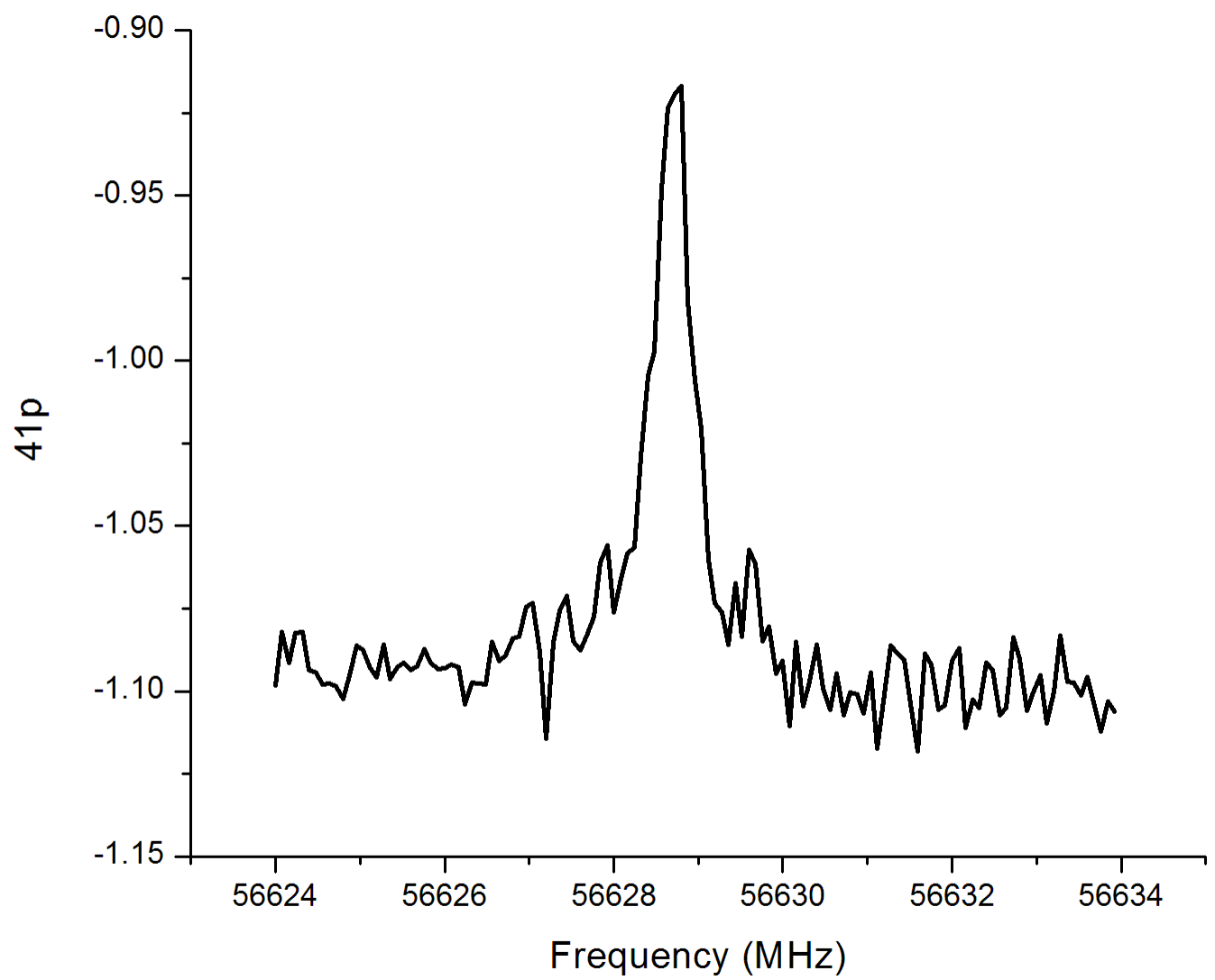

Figure 2.21: A scan over the transition from $41 s_{1 / 2} \rightarrow 41 p_{1 / 2}$ using a $2 \mu \mathrm{s}$. The lineshape shown is transform limited with a linewidth of $0.5 \mathrm{MHz}$. 
of one photon emitted from an atom falling back from $5 p_{3 / 2}$ state to $5 s_{1 / 2}$-the trapping transition. Comparing these numbers in equation 2.6 gives the approximate number of atoms $N_{M O T}$ in the MOT. The 2 in Equation 2.6 comes from the fact that roughly half of the atoms are in the $5 p_{3 / 2}$ state and half in the $5 s_{1 / 2}$ state, so the power emitted by the $5 p_{3 / 2}$ atoms emitting a photon and falling back to the $5 s_{1 / 2}$ corresponds to half of the total number of atoms. Equation 2.7 has all the values except for $\triangle P_{M O T}$ inserted in the equation. The inserted values are system-dependent and do not vary from day-to-day.

$$
\Delta \Omega=\frac{\pi r^{2}}{4 \pi R^{2}}
$$

where $R=12 \mathrm{~cm}$ is the distance from the MOT to the lens and $r=5 \mathrm{~cm}$ is the radius of the lens.

$$
P_{M O T}=\frac{\Delta P_{M O T}}{f \Delta \Omega}
$$

where $f=0.95$ is the transmission rate of the lens.

$$
E_{f}=A \frac{h c}{\lambda}
$$

where $A=3.7 * 10^{7} s^{-1}$ is Einsteins A coefficient for ${ }^{85} \mathrm{Rb} 5 p_{3 / 2}$ state, $\lambda=780 \mathrm{~nm}$ is the wavelength of the emitted photon, $h=6.6 * 10^{-34} \mathrm{Js}$ is the Planck constant, and $c=3 * 10^{8} \mathrm{~m} / \mathrm{s}$ is the speed of light.

$$
\begin{aligned}
& N_{M O T}=\frac{P_{M O T}}{2 E_{f}} \\
& N_{M O T}=\Delta P_{M O T} * 5.2 * 10^{12} W^{-1}
\end{aligned}
$$

Next we can calculate the amount of Rydberg atoms with a similar equation:

$$
N_{R y d}=\Delta P_{R y d} * 5.2 * 10^{12} W^{-1}
$$


where we need to find $\Delta P_{R y d}$. If the blue laser is allowed to excite some atoms to Rydberg states, the atoms that were excited to Rydberg states no longer absorb trap laser photons and emit photons of energy $E_{f}$ defined above. Therefore there is an decrease in the average power detected by the power meter $\left(\Delta P_{A R}\right)$ - this is not $\Delta P_{R y d}$. The typical getter current used was $4.7 \mathrm{~A}$ leading to a MOT rising time $t_{r}=0.95 \mathrm{sec}$. The blue laser excites atoms to the Rydberg state every $t_{c}=0.033$ seconds (30Hz repetition rate). Within one $33 \mathrm{~ms}$ cycle, the exponential MOT rising curve is approximately linear with a slope $\mathrm{B}=17.0 \mathrm{nW} / \mathrm{s}$. This means the MOT florescence will rise approximately a total of $t_{c} * B=0.56 \mathrm{nW}$ in between blue laser pulses. As a result:

$$
\Delta P_{R y d}=\Delta P_{A R}+0.28 \mathrm{nW}
$$

Substituting that change in power in for $\Delta P_{R y d}$ gives a rough estimate of the number of Rydberg atoms $N_{\text {Ryd }}$ shown in equation 2.8 .

Data taken on September 30th, 2011 give average values of $N_{M O T}=8.5 * 10^{4}$ atoms in the MOT and $N_{R y d}=7.7 * 10^{3}$ atoms excited to Rydberg states.

\subsubsection{Calibrating Micro-Channel Plate Detector}

While measuring the number of Rydberg atoms is important for any density calculation, it isn't ideal to use the method of the previous section any time we want the number of Rydberg atoms. For that reason, we correlate the area under the micro-channel plate detector (MCP) signal with $N_{R y d}$. For times when the density of Rydberg atoms is important, the MCP trace can be saved, the area relative to the background measured, and the number of atoms calculated from it.

A micro-channel plate detector (MCP) can be used to detect time resolved electron or ion signals. For this thesis, electrons are used since they are lighter and have a higher detection efficiency, and therefore it is possible to get a larger signal. The calibration is dependent on the MCP voltage $(1.648 \mathrm{kV})$ and the field ionization (FI) pulse voltage. The input voltage to FI circuit is a DC $\sim 40 \mathrm{~V}$ and the circuit emits a pulse of with a slope of approximately $1.3 \mathrm{kV} / \mu$ s rising to a height of $\sim 1 \mathrm{kV}$. Figure 2.22 shows an oscilloscope trace of an MCP signal with a small 41p signal and a large 41s signal. Both signals are separable and can be independently gated. If it isn't possible to distinguish 
the peaks, turn down the field ionization pulse DC voltage until they are separate. This makes the signal smaller as fewer atoms are ionized and reach the MCP detector, but the slower rising time of the slope of the FI pulse allows differentiation between the two signals (See Section 2.2.2 for more details on detection).

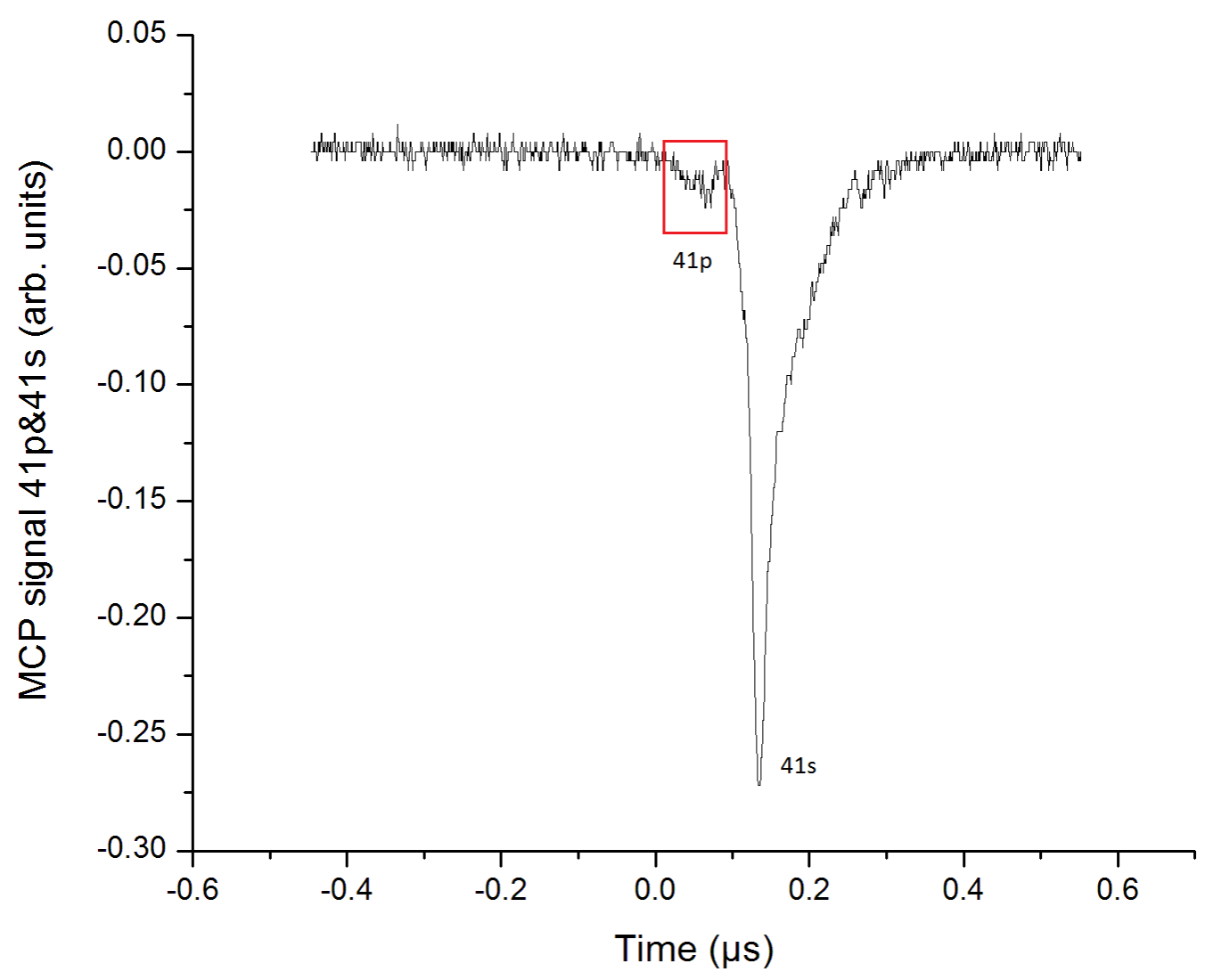

Figure 2.22: Oscilloscope scan of MCP with a small 41p signal and a large 41s signal

The $N_{R y d}$ from equation 2.8 is correlated with the area under an MCP scan to calibrate the MCP giving a factor in terms of atoms/nsV. Once a working FI voltage is decided, a scan of the Rydberg signal can be saved along with it's corresponding $\Delta P_{R y d}$ from the power meter. Then the total area under the curve is integrated, and the background subtracted. Comparing the area to the number of atoms $N_{R y d}$, it is possible to get the calibration values for the MCP. This was done for both electrons and ions and can be found in Table 2.8. Having the calibration values are very useful. However, 
as the MCP ages, it becomes less sensitive and the calibration factor changes. But provided the MCP and FI voltages aren't changed and a calibration factor was found recently, whenever we want to know the number of Rydberg atoms it is much simpler to save a MCP oscilloscope trace and integrate the area under it to find $N_{R y d}$. For daily experimentation, the power meter is replaced by a linear array detector which shows a slice of the fluorescence of the MOT. This can be used to find the MOT width and see the MOT shape and size.

\begin{tabular}{|l|l|l|l|}
\hline Particle & $\mathbf{1 n s V}=\mathbf{X}$ atoms & Error & $\mathbf{1 0 0 0}$ atom $=\mathbf{X ~ n s V}$ \\
\hline Electrons & 1000 atoms $/ \mathrm{nsV}$ & $\sim 100 \%$ & $1.0 \mathrm{nsV} / 1000$ Atoms \\
\hline Ions & 6400 atoms $/ \mathrm{nsV}$ & $\sim 100 \%$ & $0.16 \mathrm{nsV} / 1000$ Atoms \\
\hline
\end{tabular}

Table 2.8: Calibration values for MCP taken 9/30/11 with $V_{M C P}=1648 \mathrm{~V}$ and $V_{F I}=40 \mathrm{~V}$.

\subsubsection{Width of MOT}

Knowing the width of the MOT is necessary to calculate the density of the MOT. The MOT width can be viewed through a CCD linear array detector in the same position as the power meter in the previous section. Figure 2.23 is an example of what the MOT looked like on the linear array. Since the mirrors that control the trap and repump lasers drift out of alignment with time, it is necessary to regularly realign the MOT. While the MOT is generally within a range of typical widths, for any time when a density measurement matters, the width is recorded. Table 2.9 shows some example widths as a reference.

\begin{tabular}{|l|l|l|}
\hline Date & MOT width $(\mu s)$ & MOT FWHM $(\mu m)$ \\
\hline $12-19-11$ & 128 & 860 \\
\hline $5-30-12$ & 130 & 870 \\
\hline $6-13-12$ & 180 & 1200 \\
\hline
\end{tabular}

Table 2.9: Widths of the MOT taken on different days

The linear array detector is placed on a 3D translation stage in front of a lens to collect emitted radiation from the MOT. The translation stage allows us to align the detector so that it is centered on the MOT. The detector outputs both a signal and a trigger to an oscilloscope. The simplest way to tell the MOT signal from other fluorescence sources is by blocking any of the MOT lasers. The MOT will disappear and reform once the laser is unblocked. The width of the MOT refers to the full width at half maximum (FWHM) of the MOT radiation signal. Assuming the MOT is spherical 


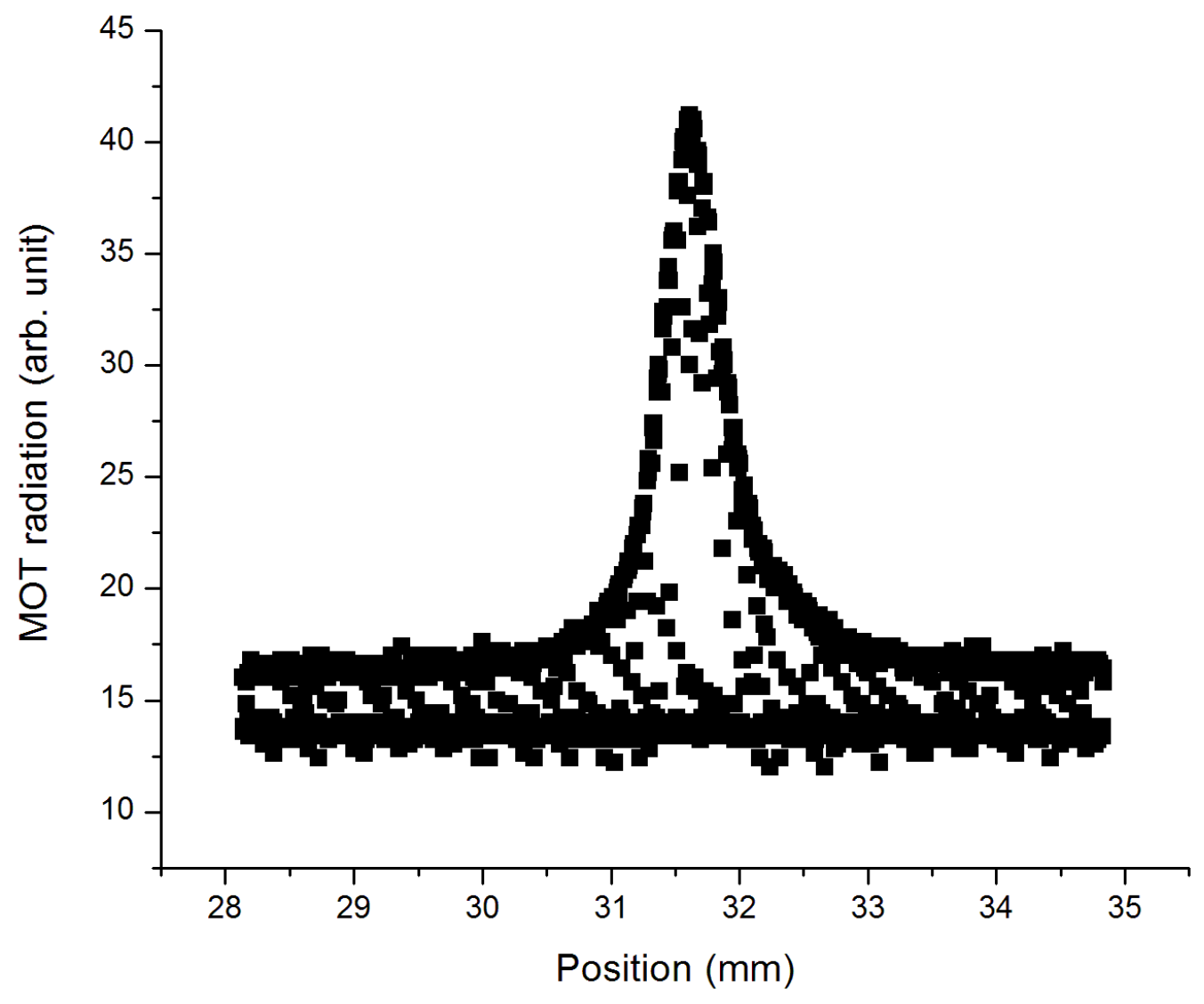

Figure 2.23: MOT signal viewed through linear array detector on 6-13-12, FWHM $=1.2 \mu \mathrm{m}$. 
and the linear array is detecting the middle of the MOT, this is also the diameter of the MOT from which we can calculate the volume of the MOT and from there the density. The linear array signal is also useful for viewing the MOT during mirror optimization.

Neutral density filters are used to prevent saturation of the linear array. On the scope, the scale is in ms. There are a number of calibration factors to convert the width (FWHM) on a oscilloscope screen $\left(t_{r}\right)$ to the physical MOT width $\left(w_{M O T}\right)$. These are shown in Equation 2.10. The first conversion factor $C_{1}$ changes from ms to pixels. The second, $C_{2}$ converts from pixels to millimeters. $C_{1}$ and $C_{2}$ both are specifications of the linear array detector. The third conversion factor, $m_{x}$ is the magnification due to the lens and is setup dependent. The MOT's emitted light that passes through the lens forms an image on the detector. The conversion factor for the imaged MOT can be found using equation 2.11.

$$
\begin{aligned}
\begin{aligned}
& w_{M O T}=t_{r} * C_{1} * C_{2} * m_{x} \\
&=\left(\frac{1}{5 * 10^{-} 3 \mathrm{~ms} / \text { pixel }}\right) *(0.014 \mathrm{~mm} / \text { pixel }) * 2.4 * t_{r}(\mathrm{~ms}) \\
& w_{M O T}=6.72 \mathrm{~mm} / \mathrm{ms} * t_{r}(\mathrm{~ms}) \\
& m_{x}=\frac{n s^{\prime}}{n^{\prime} s}
\end{aligned}
\end{aligned}
$$

where $s$ refers to the distance from the object (the MOT) to the lens, $s^{\prime}$ refers to the distance from lens to the image, and $n$ and $n^{\prime}$ refer to the index of reflection on the object and image side of the lens respectively.

\subsubsection{Density}

\section{Density of MOT}

The average density of the MOT $\left(\rho_{M O T}\right)$ can be found using the width of the MOT $\left(w_{M O T}\right.$ defined in equation 2.10) and the number of atoms $\left(N_{M O T}\right.$ defined in equation 2.7). The MOT is roughly 
spherical so the MOT width can be used to calculate a spherical volume.

$$
\rho_{M O T}=\frac{N_{M O T}}{\frac{4}{3} \pi\left(w_{M O T} / 2\right)^{3}}
$$

This assumes the density is constant; a better assumption would be the density of the MOT is a Gaussian distribution [2]. However, that isn't necessary in this thesis.

\section{Density of Rydberg atoms}

Rydberg atoms are excited by the 480nm light passing through the center of the MOT. Because of this the volume of excited atoms is cylindrical in shape and can be calculated using Equation 2.13.

$$
\rho_{M O T}=\frac{N_{R y d}}{\left(w_{M O T} / 2\right) \pi r_{b}^{2}}
$$

where $N_{R y d}$ is the number of Rydberg atoms calculated with equation $2.8, w_{M O T}$ is the width of the MOT from equation 2.10, and $r_{b} \approx 0.16 \mathrm{~mm}$ [2] is the radius of the blue laser exciting the atoms to Rydberg states.

The density is important because inter-atomic interactions are separation dependent. Higher densities correspond to stronger interactions. It's important to remember that this is an average density. Calculating the actual density at different points in the MOT can be done by assuming the density is a Gaussian distribution.

\subsubsection{MOT Rise Time}

When any one of the lasers or the magnetic field is blocked, the MOT disperses. When all necessary components for the MOT return, it will reform within a couple seconds. The speed at which it fills is largely dependent on the number of Rubidium atoms in the vacuum chamber. This is controlled by using getters. Current is sent through the getter causing atoms to boil off. An ion pump runs continuously to keep the chamber at vacuum of around $10^{-9}$ Torr. Between the two, there is a different stable amount of rubidium atoms available in the chamber for each setting of getter current. In addition, as the getters age, they need higher currents to provide the same amount of 
atoms.

Since atoms in the MOT are continuously being excited to the $5 p_{3 / 2}$ state and then emitting a photon and falling back to the $5 s_{1 / 2}$ state, the atoms in the MOT fluoresce provide a good measure of the number of atoms in the MOT. The MOT filling back up to stable size after being dispersed (say through blocking a beam) can be viewed through the photodiode placed behind a lens. Figure 2.24 shows the MOT filling for three different getter currents. The rise time is found by fitting the curves of the MOT refilling to exponential curves, Equation 2.14. The rise time for the exponential, $t_{r}$, is the MOT's filling time. The results are summarized in Table 2.10. This data were taken on September 30th, 2012. Since the getters decay with use, the rise time will increase and maximum total population will decrease for a given current with time.

$$
y=A m p * e^{x / t_{r}}+y_{0}
$$

\begin{tabular}{|l|l|l|}
\hline Getter current $(\mathbf{A})$ & Rise Time, $t_{r}$ (sec) & Std. Dev. (sec) \\
\hline 4.0 & 1.99 & 0.15 \\
\hline 4.4 & 1.38 & 0.10 \\
\hline 4.7 & 0.95 & 0.12 \\
\hline
\end{tabular}

Table 2.10: Summary of rise time values for the filling of the MOT taken September 30, 2011. These values are probably no longer accurate as the getters have been used a great deal since then. 


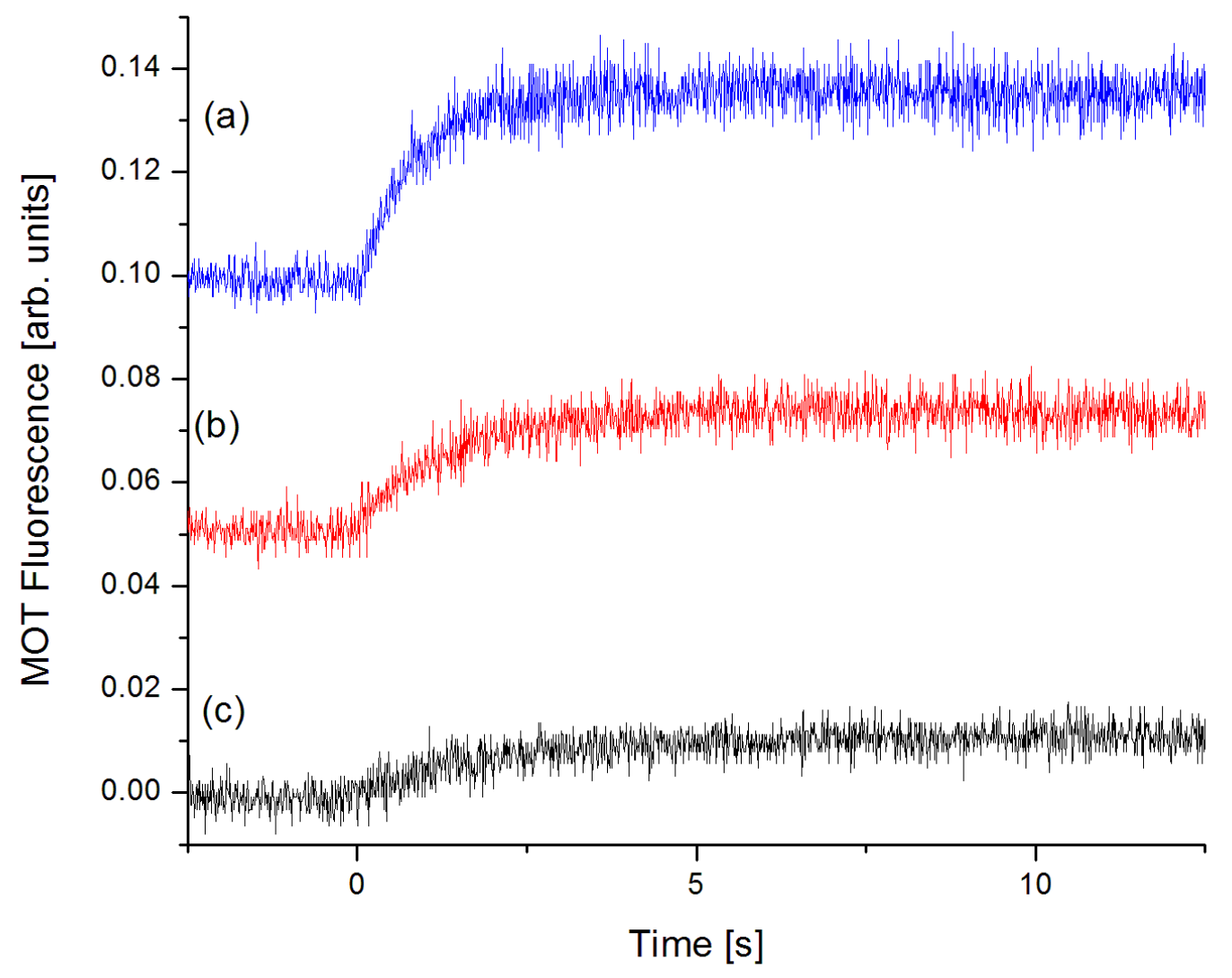

Figure 2.24: MOT filling time for different getter currents. Initially the trap laser is blocked, and when it is unblocked the MOT starts to fill and emit radiation. a) Getter current $=4.7 \mathrm{~A}$, Rise time $=0.95 \mathrm{sec} \pm 0.12 \mathrm{~b}$ ) Getter current $=4.4 \mathrm{~A}$, Rise time $=1.38 \mathrm{sec} \pm 0.10 \mathrm{c}$ ) Getter current $=4.0$ A, Rise time $=1.99 \mathrm{sec} \pm 0.15$. 


\subsection{Nulling Stray Fields}

\subsubsection{Motivation}

There are two main reasons to null all external magnetic fields. The first is that Rydberg states are sensitive to external fields [1]. Even after the MOT magnetic field is switched off (see Section 2.1.4), there are still stray fields from the earth's magnetic field and other sources that can effect the experiment. The second reason is that when the trap field is turned off the trap moves if there is a nonzero residual magnetic field. These external fields must be nulled using three sets of Helmholtz coils so it is possible to see changes in the transition linewidths from atomic interactions and controlled fields with microwave spectroscopy. The Helmholtz coils are set on three perpendicular axes and are capable of nulling stray fields to the limit of the detection method. However, doing so is at the limit of tuning the current supplies which control the Helmholtz coils. It requires minimal turns of the control dials beyond the display sensitivity. For example on the $\mathrm{z}$ axis a current of $111 \mathrm{~mA}$ seems to work best. However, the display for the current controller has marks only every $5 \mathrm{~mA}$, and current changes of what can be estimated to be $\sim 1 \mathrm{~mA}$ affect the lineshape in a small but visible fashion.

As discussed in Section 2.3, microwave transitions can be broadened for a number of reasons. One of those sources of broadening is stray fields. Once all other sources of broadening have been controlled for, it is possible to use the microwave transition width as a probe of stray fields.

In addition to seeing if all stray fields are nulled, this provides a general check to see if everything is working. If timing for the field ionization pulse or the magnetic field switching is wrong, the transition lineshape will broaden. See Section 2.5.3 for common problems.

\subsubsection{Experimental Procedure}

To null all stray external magnetic fields, the transition linewidth of $41 s_{1 / 2} \rightarrow 41 p_{1 / 2}$ is minimized in frequency space. Figure 2.25 shows an energy level diagram for the transition used. A microwave pulse is scanned over the frequency resonance and fitted to a Lorentzian to find the width.

Here is a brief outline of how the experiment works. First the MOT magnetic field is switched off, and after a $5 \mathrm{~ms}$ delay atoms are excited to the $41 s_{1 / 2}$ Rydberg state at low density. Next a microwave pulse excites the atoms to $41 p_{1 / 2}$, and finally the atoms are field ionized to detect 
the number of atoms in the $41 s_{1 / 2}$ and $41 p_{1 / 2}$ states on the MCP. See Figure 2.26 for the timing. The $41 p_{1 / 2}$ signal is gated by the boxcar integrator and for each laser shot the signal area is sent to the computer. After a predetermined number of shots are averaged, the computer controlling the microwave synthesizer changes the frequency by a chosen step size. Through this method the desired frequency range is scanned. The lower limit of the width of a resonance in frequency space is the transform of the pulse length $\tau_{M W}$, i.e. $w_{m i n}=\tau_{M W}^{-1}$. So an $2 \mu s$ pulse will have a minimum linewidth of $0.5 \mathrm{MHz}$ and an $1 \mu s$ pulse will have a minimum linewidth of $1 \mathrm{MHz}$.

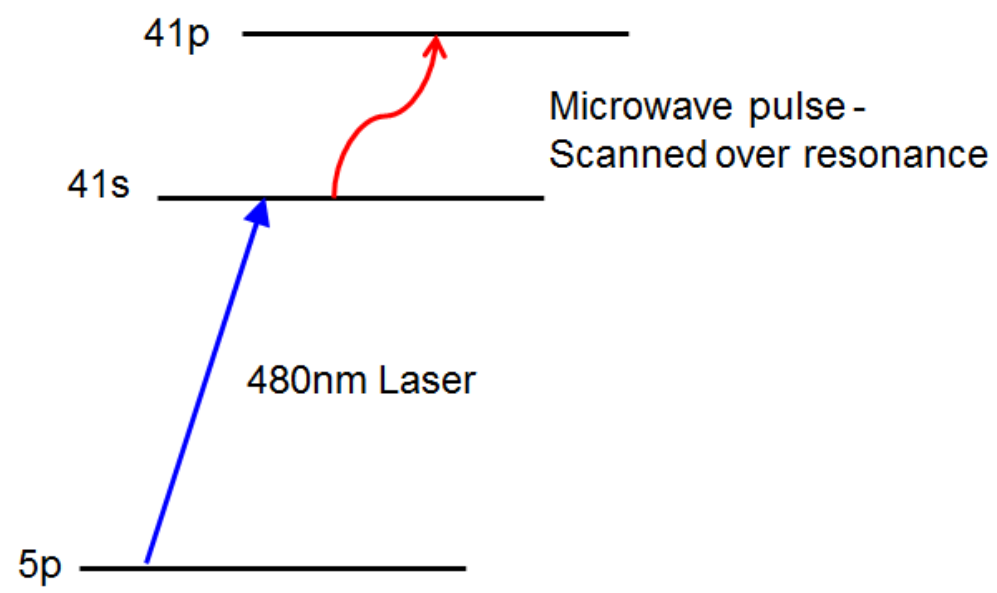

Figure 2.25: Diagram of energy levels for nulling experiment. The blue $(\sim 480 \mathrm{~nm})$ laser is used to excite the trapped atoms to a Rydberg state, and then a microwave field pulse is scanned over the $41 \mathrm{~s} \rightarrow 41 \mathrm{p}$ transition.

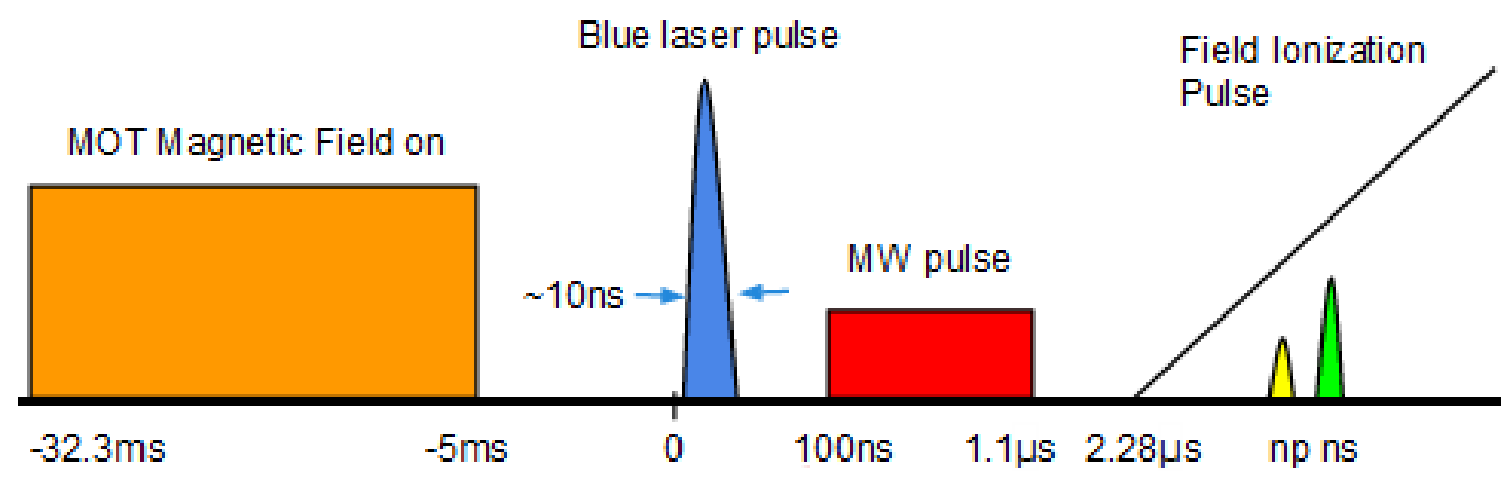

Figure 2.26: Timing diagram for nulling stray fields by probing a microwave transition.

The experiment should be started with low Rydberg atom population, low microwave power, 
and the MOT magnetic field switching on (see Section 2.1.4). However even taking those thing into account, without nulling coils and using a $1 \mu$ s pulse, the linewidth may be over three times wider than the transform limited linewidth. There are three sets of magnetic coils to null external magnetic fields on three orthogonal axes. The axis perpendicular to the optics table is defined as the $\mathrm{z}$-axis. The $\mathrm{x}$-axis and $\mathrm{y}$-axis are arbitrarily defined and labeled at their current controllers. Starting with the z-axis, the current can be varied in $10 \mathrm{~mA}$ steps, taking multiple microwave scans at each current. The transition widths can be fitted to Lorentzian curves. The width will decrease as the magnetic field created by the Helmholtz coils cancels the stray fields on that axis. At some point the width will plateau at a minimum determined by the pulse length. By scanning beyond this plateau in both directions it is possible to find the center of it. Setting the current for the z-axis at the center of the plateau, it is possible to move on to the next axis. After all three axes have been completed, this roughly nulls the stray fields. See Figure 2.27 for graphs with one of the axes not nulled as compared to the graph with all axes nulled. The z-axis nulling has the greatest effect on the broadening and asymmetry because the Earth's fields are strongest in that direction.

For fine adjustments, one can switch to a longer microwave pulse - it will have a smaller transformlimited linewidth. For the final tweaking, the microwave attenuation is decreased. The broadening from higher microwave power can greatly exaggerate any asymmetries in the peak from stray fields. Adjusting the current until it is symmetric (or as close as possible) results in the stray fields being nulled to the limit of this detection method. This requires minute adjustments of the current supplies for the Helmholtz coils. Also, day-to-day changes in the earth's magnetic field as well as other sources of stray fields in the area mean that this sort of asymmetry can reappear. Table 2.11 contains the nulling currents used. These are specific to this particular experimental setup. For the $\mathrm{x}$-axis current controller, the voltage displayed can be used to get additional sensitivity on the current it is supplying.

\begin{tabular}{|l|l|l|}
\hline$I_{x}$ & $I_{y}$ & $I_{z}$ \\
\hline $40 \mathrm{~mA}(0.10 \mathrm{~V})$ & $6 \mathrm{~mA}$ & $111 \mathrm{~mA}$ \\
\hline
\end{tabular}

Table 2.11: Final nulling currents used to null all stray fields.

Another potential method to check that all stray fields are nulled would involve viewing either Rabi features (small side lobes on the MW transition) or Ramsey fringes (uses 2 microwave pulses 


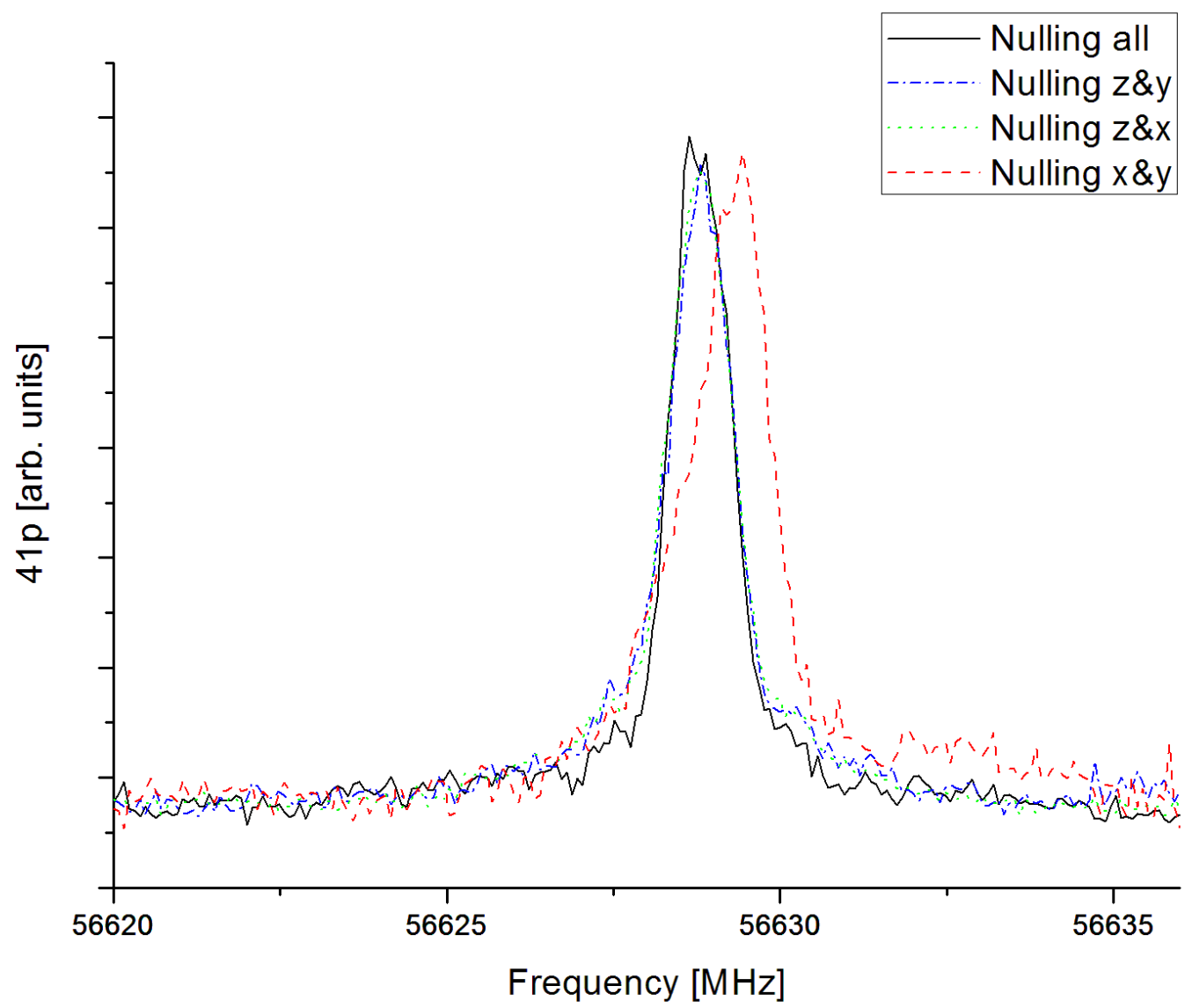

Figure 2.27: Stray fields are nulled through Helmholtz coils on three axis. The largest field is on the $\mathrm{z}$ axis as can be seen by the largest broadening results from not nulling fields on that axis. 
which interfere instead of one) [2]. However, getting a transform-limited linewidth for a $2 \mu s$ pulse was considered sufficient for the purposes of this thesis. Rabi features were viewed, particularly when the microwave power is increased to exaggerate them, but since they are small relative to the main signal and the noise it's difficult to make conclusive comments regarding them.

\subsubsection{Troubleshooting}

There are a number of sources of broadening for the lineshape. Table 2.12 contains the most common and how to fix them. Since the microwave transition linewidth is sensitive to many things in the experimental setup, it can be an excellent check that everything is working well or a way to figure out what is not working. Another common source of problems is the various cords and their connections going bad.

\begin{tabular}{|l|l|l|l|}
\hline Source & Type & Troubleshoot & Graphs \\
\hline MW probe power & Symmetric & lower power until linewidth minimized & 2.29 \\
\hline \multirow{2}{*}{ Stray fields } & \multirow{2}{*}{ Asymmetric } & Adjust Helmholtz coils current & 2.27 \\
\cline { 3 - 4 } & & check magnetic field switching & 2.9 \\
\cline { 3 - 4 } & & FI and MW pulses overlap & 2.28 \\
\hline Rydberg density & Symmetric & attenuate blue laser & $3.16 \mathrm{~b}$ \\
\hline Rabi Oscillation & dip at resonance & change attenuation by a small amount & N/A \\
\hline Background & Irregular & trigger off AC line or average scans & 2.13 \\
\hline Transform limit & (MW pulse period) $^{-1}$ & Increase MW pulse length & 2.21 \\
\hline
\end{tabular}

Table 2.12: Different types of microwave transition broadening and other problems 


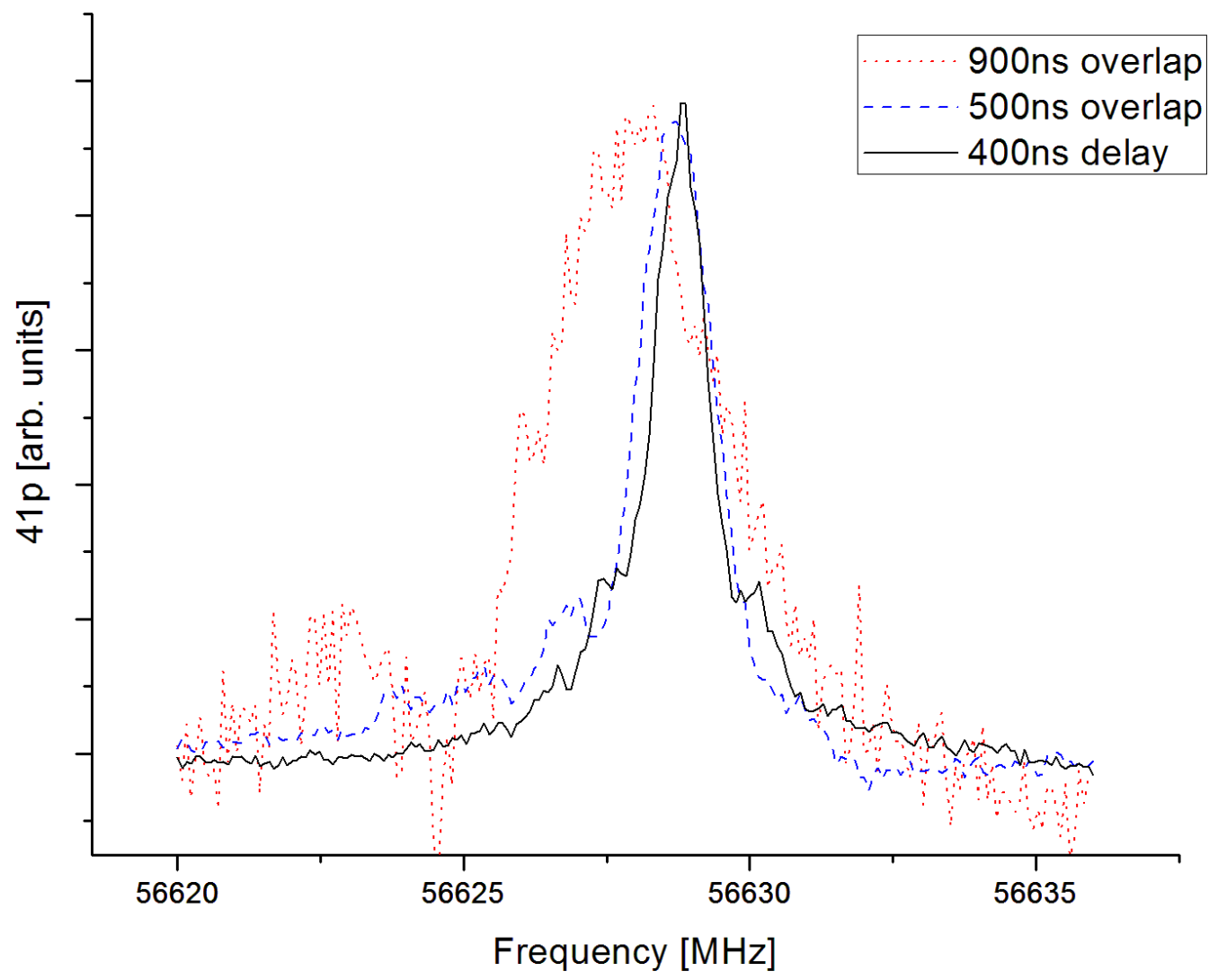

Figure 2.28: These curves show why it's important for the timing diagrams to be followed, when the MW pulse and the FI pulse overlap, asymmetries and broadening occur. 


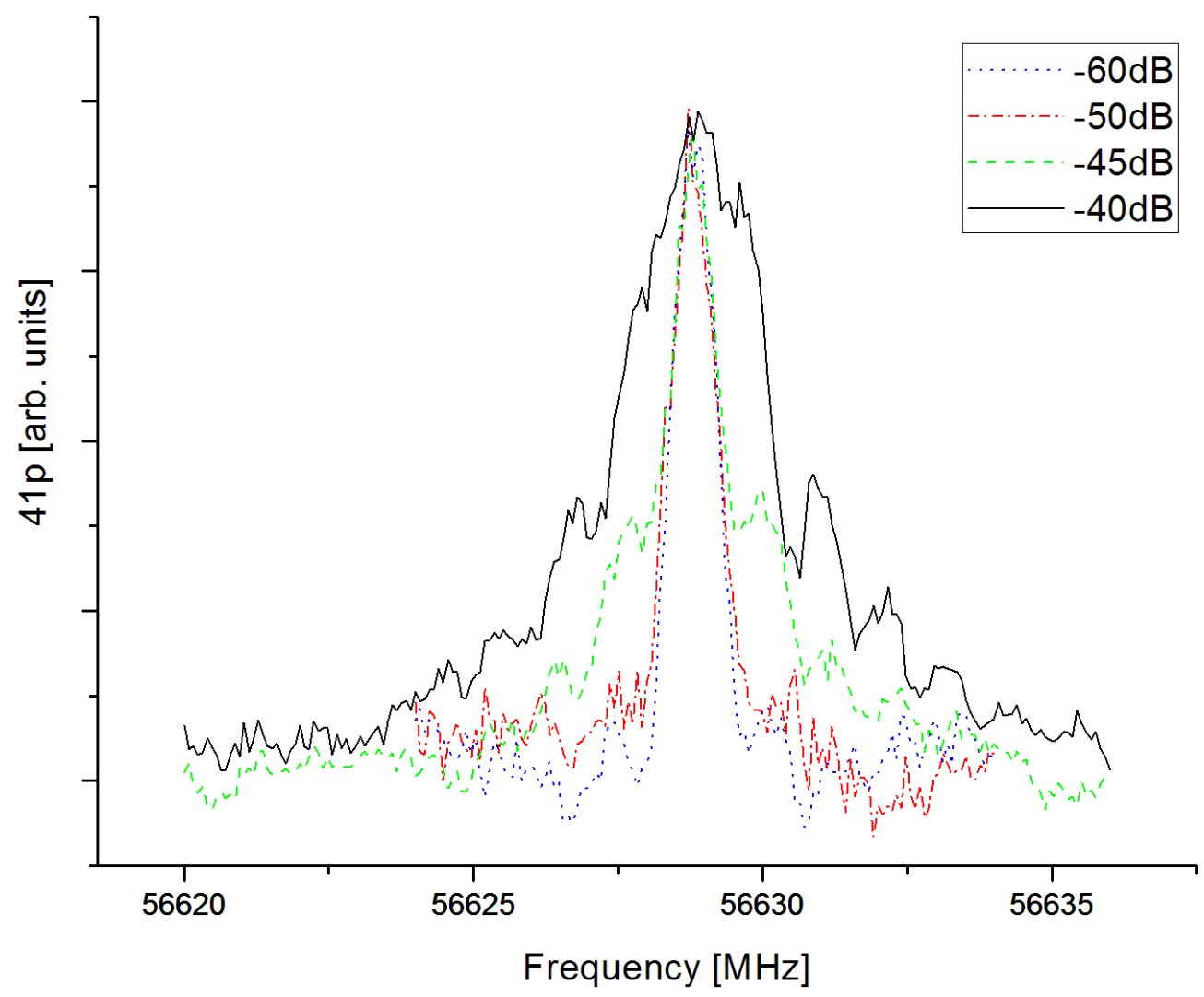

Figure 2.29: Decreasing the attenuation on the MW probe beam increase the power causing broadening. This also causes some features such as side lobes and asymmetries to become more pronounced 


\section{Chapter 3}

\section{Dressed State Experiment}

\subsection{Introduction}

Dressed state atoms are created through the coupling of two states by a dressing microwave pulse. This experiment explores the dressed state $41 p_{1 / 2}+42 s_{1 / 2}$. The original goal of this experiment was to study dipole-dipole interactions at different densities of Rydberg atoms. However, that goal changed when instead of two dressed states we viewed four. This changed the primary purpose of this experiment to documenting and explaining the dependence of the dressed states on the dressing microwave field.

This chapter will begin with theory deriving the expected behavior of the dressed states in Section 3.2. In Section 3.3 we discuss the specific experimental approach used, and Section 3.4 discusses the results and analysis.

\subsection{Dressed State Theory}

In this section we calculate the Hamiltonian matrix for the dressed state; this allows us to calculate eigenvalues for different initial conditions. The eigenvalues generated for the energy of the dressed state are related to the center frequencies of the peaks found in microwave scans in the experiment. This is the basis for the analysis of the data in Section 3.4. 


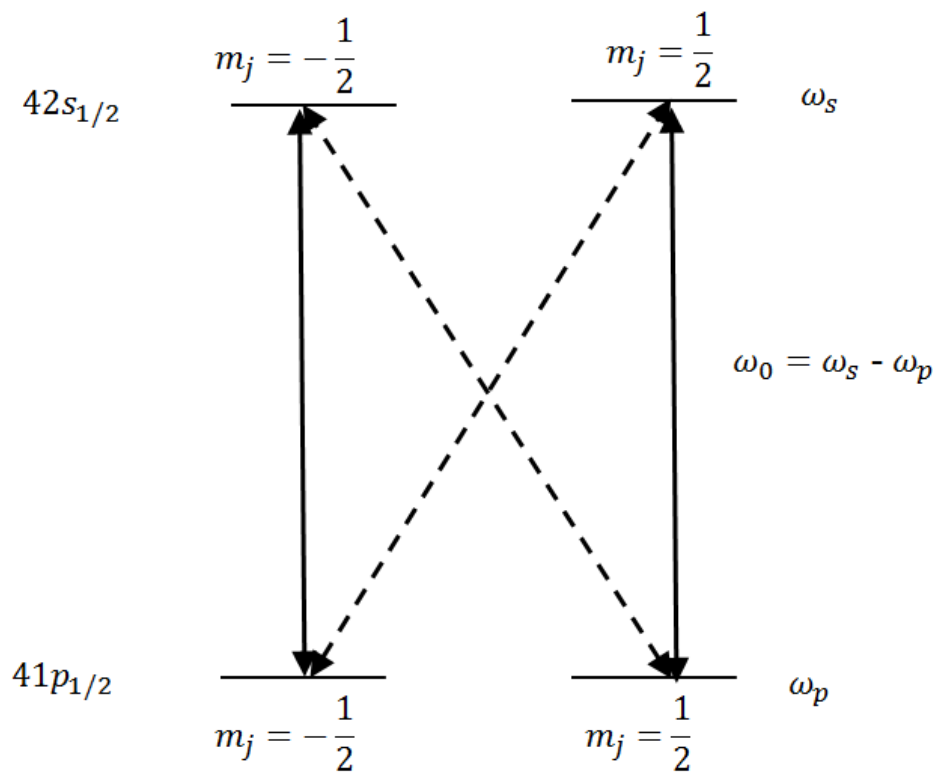

Figure 3.1: Diagram of the levels coupled in the dressed state. The solid arrows lines show coupling via field polarized in the $\hat{z}$ direction and the dotted arrow lines show coupling via field polarized in the $\hat{x}$ direction

We have the four level system pictured in Figure 3.4. We can define a state $F(t)$ as:

$$
F(t)=a_{1}(t)|42 s,+1 / 2\rangle+a_{2}(t)|42 s,-1 / 2\rangle+a_{3}(t)|41 p,+1 / 2\rangle+a_{4}(t)|41 p,-1 / 2\rangle
$$

where $F(t)$ is the solution to the time dependent Schroedinger equation:

$$
i \frac{d F(t)}{d t}=\mathcal{H} F(t)
$$

where $\mathcal{H}$ is the system Hamiltonian. For a system without the dressing field interaction the Hamiltonian is:

$$
\mathcal{H}_{0}=\left[\begin{array}{llll}
\omega_{1} & & & \\
& \omega_{2} & & \\
& & \omega_{3} & \\
& & & \omega_{4}
\end{array}\right]=\left[\begin{array}{llll}
\omega_{s} & & & \\
& \omega_{s} & & \\
& & \omega_{p} & \\
& & & \omega_{p}
\end{array}\right]
$$


where $\omega_{s}$ and $\omega_{p}$ are the resonance frequencies between that state and a chosen reference point. We shall use $41 s_{1 / 2}$ as the reference point because that will make $\omega_{p}$ equal to the unshifted resonant transition between $41 s_{1 / 2}$ and $41 p_{1 / 2}$ which we will later use experimentally to probe the dressed state. There is a degeneracy between the $m_{j}= \pm 1 / 2$ levels.

However, we also have interactions between the states arising from the dressing field. They are defined by the Hamiltonian:

$$
\mathcal{H}_{d}=e \vec{r} \cdot \vec{E}_{d}(t)
$$

where $e=1$ a.u. is the charge of an electron and $\vec{E}_{d}(t)$ is the dressing field.

This means the Hamiltonian for the system is:

$$
\mathcal{H}=\mathcal{H}_{0}+\mathcal{H}_{d}
$$

The dressing field emitted from the horn is linearly polarized at each point so naively we would assume our dressing field is the same. However, the data will show there is an elliptical component of varying amplitude, so we will calculate the matrix for a elliptical field and then set $g=0$ to see the linear case. Our coupling electric field is then:

$$
\vec{E}_{d}(t)=E(\hat{z} \cos (\omega t)+\hat{x} g \cos (\omega t+\phi))
$$

where $\omega$ is the field frequency. The $\hat{z}$ direction is chosen to be in the direction of the strongest field and $E$ is the magnitude in that direction. $\hat{x}$ is a second orthogonal axis of polarization. Let $g=E_{x} / E$ and $\phi=$ the phase difference between polarizations. Since $\hat{z}$ is determined by the direction of the strongest field, $g$ will always be less than 1 . The field position dependence is wrapped into the definition of $E$ and $g$.

This gives us a dressing field Hamiltonian of:

$$
\mathcal{H}_{d}=E(z \cos (\omega t)+x g \cos (\omega t+\phi))
$$


The non-zero matrix elements of $\mathcal{H}_{d}$ are:

$$
\begin{aligned}
& m_{1}(t)=E\langle 42 s,+1 / 2(t)|z \cos (\omega t)| 41 p,+1 / 2(t)\rangle \\
& m_{2}(t)=E\langle 42 s,-1 / 2(t)|z \cos (\omega t)| 41 p,-1 / 2(t)\rangle \\
& m_{3}(t)=E\langle 42 s,+1 / 2(t)|x g \cos (\omega t+\phi)| 41 p,-1 / 2(t)\rangle \\
& m_{4}(t)=E\langle 42 s,-1 / 2(t)|x g \cos (\omega t+\phi)| 41 p,+1 / 2(t)\rangle
\end{aligned}
$$

where $|\alpha(t)\rangle$ refers to the fact that the states have a time dependence. Therefore, the matrix representing $\mathcal{H}=\mathcal{H}_{0}+\mathcal{H}_{d}$ gives:

$$
\mathcal{H}=\left[\begin{array}{cccc}
\omega_{p} & & m_{1}(t) & m_{3}(t) \\
& \omega_{p} & m_{4}(t) & m_{2}(t) \\
m_{1}(t) & m_{4}(t) & \omega_{s} & \\
m_{3}(t) & m_{2}(t) & & \omega_{s}
\end{array}\right]
$$

To tie these matrix elements back to Figure 3.4, the matrix elements $m_{1}(t)$ and $m_{2}(t)$ use the field polarized in the $\hat{z}$ direction to couple states with the same $m_{j}$ and are represented by the solid line. The matrix elements $m_{3}(t)$ and $m_{4}(t)$ couple states with different $m_{j}$ using the field polarized in the $\hat{x}$ direction and are represented by the dashed line. $\omega_{s}$ and $\omega_{p}$ are the unshifted energy levels of $42 s_{1 / 2}$ and $41 p_{1 / 2}$ respectively.

The time dependent Schroedinger equation in matrix notation is:

$$
i \frac{d}{d t}\left[\begin{array}{l}
a_{1}(t) \\
a_{2}(t) \\
a_{3}(t) \\
a_{4}(t)
\end{array}\right]=\left[\begin{array}{cccc}
\omega_{s} & & m_{1}(t) & m_{3}(t) \\
& \omega_{s} & m_{4}(t) & m_{2}(t) \\
m_{1}(t) & m_{4}(t) & \omega_{p} & \\
m_{3}(t) & m_{2}(t) & & \omega_{p}
\end{array}\right]\left[\begin{array}{l}
a_{1}(t) \\
a_{2}(t) \\
a_{3}(t) \\
a_{4}(t)
\end{array}\right]
$$

where $a_{\alpha}(t), \alpha=1,2,3,4$ is defined by $F(t)$ in equation 3.1

The next step is to use Floquet theory from Shirley's paper "Solution of the Schroedinger equation with a Hamiltonian periodic in time" [8] to write an approximately equivalent time independent Hamiltonian. Once we have a time independent Hamiltonian, it's possible to find the energy eigenvalues for the dressed state. We will then calculate the different matrix elements based on the 
dressing field. The eigenvalues can be found as a function of the field and it is possible to explore the effects of the field on the energy eigenvalues.

\subsubsection{Floquet Theory}

Floquet theory equates the time dependent $\mathcal{H}$ with a time independent infinite matrix $\mathcal{H}_{F}$. We can then take a $4 \mathrm{p} \mathrm{x} 4 \mathrm{p}$ matrix $(4 \mathrm{x} 4,8 \mathrm{x} 8,12 \mathrm{x} 12$, etc) from the infinite Floquet matrix and find the eigenvalues for it. The dimensions need to be a multiple of four since we have a four level system. An $\mathrm{N}$ level system has $\mathrm{N}$ meaningful eigenvalues. The larger eigenvalues modulo $\omega$ will equal one of the $\mathrm{N}$ smallest eigenvalues [8]. The larger the matrix, the better the approximation is. We will use the $4 \mathrm{x} 4$ matrix, equivalent to taking the rotating wave approximation. If we were to use the $8 \mathrm{x} 8$ matrix centered on our 4x4 matrix, it would include the Bloch-Siegert shift [8]. However, that isn't necessary for our problem.

To apply Floquet theory, we first need to separate the time dependence in the matrix element from the time independent parts. Let's define:

$$
\begin{aligned}
& m_{1}(t)=E \cos (\omega t)\langle 42 s+1 / 2(t)|z| 41 p+1 / 2(t)\rangle=M_{1} \cos (\omega t) \\
& m_{2}(t)=E \cos (\omega t)\langle 42 s-1 / 2(t)|z| 41 p-1 / 2(t)\rangle=M_{2} \cos (\omega t) \\
& m_{3}(t)=E g \cos (\omega t+\phi)\langle 42 s+1 / 2(t)|x| 41 p-1 / 2(t)\rangle=M_{3} \cos (\omega t+\phi) \\
& m_{4}(t)=E g \cos (\omega t+\phi)\langle 42 s-1 / 2(t)|x| 41 p+1 / 2(t)\rangle=M_{4} \cos (\omega t+\phi)
\end{aligned}
$$

and the time dependence of the states can be written as [8]:

$$
a_{\alpha}(t)=\sum_{n=-\infty}^{\infty} a_{\alpha}^{(n)} e^{i n \omega t}
$$


Explicitly writing the time dependence in the Schroedinger equation gives:

$$
\begin{aligned}
& i \frac{d}{d t}\left[\begin{array}{l}
\sum a_{1}^{(n)} e^{i n \omega t} \\
\sum a_{2}^{(n)} e^{i n \omega t} \\
\sum a_{3}^{(n)} e^{i n \omega t} \\
\sum a_{4}^{(n)} e^{i n \omega t}
\end{array}\right] \\
& =\left[\begin{array}{cccc}
\omega_{p} & & M_{1} \cos (\omega t) & M_{3} \cos (\omega t+\phi) \\
M_{1} \cos (\omega t) & M_{3} \cos (\omega t+\phi) & M_{4} \cos (\omega t+\phi) & M_{2} \cos (\omega t) \\
M_{3} \cos (\omega t+\phi) & M_{2} \cos (\omega t) & & \\
\sum a_{2}^{(n)} e^{i n \omega t} \\
\sum a_{3}^{(n)} e^{i n \omega t} \\
\sum a_{4}^{(n)} e^{i n \omega t}
\end{array}\right]
\end{aligned}
$$

Writing out the first equation of the matrix equation in Equation 3.13 gives:

$$
\begin{aligned}
i \sum & (i n \omega) a_{1}^{(n)} e^{i n \omega t}=\sum \omega_{1} a_{1}^{(n)} e^{i n \omega t}+M_{1} \cos \omega t \sum a_{3}^{(n)} e^{i n \omega t} \\
& +M_{3} \cos (\omega t+\phi) \sum a_{4}^{(n)} e^{i n \omega t} \\
= & \sum \omega_{1} a_{1}^{(n)} e^{i n \omega t}+\frac{M_{1}}{2} \sum a_{3}^{(n)} e^{i(n-1) \omega t} \\
& +\frac{M_{1}}{2} \sum a_{3}^{(n)} e^{i(n+1) \omega t}+\frac{M_{3}}{2} \sum a_{4}^{(n)} e^{i(n-1) \omega t-i \phi}+\frac{M_{3}}{2} \sum a_{4}^{(n)} e^{i(n+1) \omega t+i \phi}
\end{aligned}
$$

which can be simplified to:

$$
0=\left(n \omega+\omega_{p}\right) a_{1}^{(n)}+\frac{M_{1}}{2} a_{3}^{(n+1)}+\frac{M_{1}}{2} a_{3}^{(n-1)}+\frac{M_{3}}{2} a_{4}^{(n+1)} e^{-i \phi}+\frac{M_{3}}{2} a_{4}^{(n-1)} e^{i \phi}
$$

for all $\mathrm{n}$.

This can be done for the other 3 equations and summarized in an infinite Floquet matrix shown 
for $n=0,-1$ below [8]:

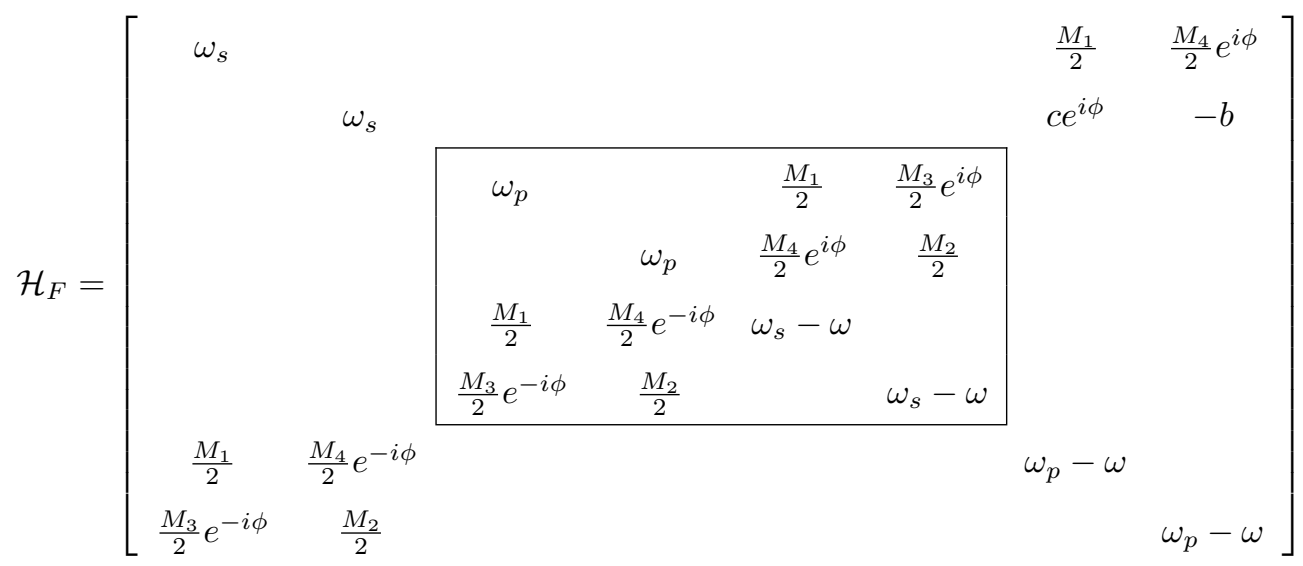

In practice we don't calculate the eigenvalues for the infinite matrix. While there are an infinite number of Floquet energies (or quasi energies) there are only 4 meaningful ones. If we had $\mathrm{N}$ states there would be $\mathrm{N}$ meaningful energies. All quasi energies are the 4 smallest quasi energies modulo $\omega$ i.e. [8]:

$$
\lambda_{\alpha, p}=\lambda_{\alpha, 0}+p \omega
$$

where $\alpha$ can be 1 though $4, \lambda_{\alpha, 0}$ is one of the smallest absolute value quasi energies, and $p$ is an integer.

As mentioned earlier, we approximate our Floquet matrix to the $4 \mathrm{x} 4$ matrix outlined in equation 3.16.

$$
\mathcal{H}_{F}={ }_{p_{-1 / 2}}^{p_{1 / 2}} s_{1 / 2}\left[\begin{array}{cccc}
p_{1 / 2} & p_{-1 / 2} & s_{1 / 2} & s_{-1 / 2} \\
s_{-1 / 2} & 0 & \frac{M_{1}}{2} & \frac{M_{3}}{2} e^{i \phi} \\
0 & \omega_{p} & \frac{M_{4}}{2} e^{i \phi} & \frac{M_{2}}{2} \\
\frac{M_{1}}{2} & \frac{M_{4}}{2} e^{-i \phi} & \omega_{s}-\omega & 0 \\
\frac{M_{3}}{2} e^{-i \phi} & \frac{M_{2}}{2} & 0 & \omega_{s}-\omega
\end{array}\right]
$$

Next we define $\omega_{0}=\omega_{s}-\omega_{p}$ as the resonant frequency between the $41 p_{1 / 2}$ and $42 s_{1 / 2}$ states. 
Substituting that into the matrix gives:

$$
\begin{aligned}
\mathcal{H}_{F} & =\left[\begin{array}{cccc}
\omega_{p} & 0 & \frac{M_{1}}{2} & \frac{M_{3}}{2} e^{i \phi} \\
0 & \omega_{p} & \frac{M_{4}}{2} e^{i \phi} & \frac{M_{2}}{2} \\
\frac{M_{1}}{2} & \frac{M_{4}}{2} e^{-i \phi} & \omega_{0}-\omega+\omega_{p} & 0 \\
\frac{M_{3}}{2} e^{-i \phi} & \frac{M_{2}}{2} & 0 & \omega_{0}-\omega+\omega_{p}
\end{array}\right] \\
& =\left[\begin{array}{cccc}
0 & 0 & \frac{M_{1}}{2} & \frac{M_{3}}{2} e^{i \phi} \\
0 & 0 & \frac{M_{4}}{2} e^{i \phi} & \frac{M_{2}}{2} \\
\frac{M_{1}}{2} & \frac{M_{4}}{2} e^{-i \phi} & \omega_{0}-\omega & 0 \\
\frac{M_{3}}{2} e^{-i \phi} & \frac{M_{2}}{2} & 0 & \omega_{0}-\omega
\end{array}\right]+\omega_{p} \mathcal{I}
\end{aligned}
$$

where $\mathcal{I}$ is the identity matrix. Define $\delta=\omega-\omega_{0}$ as the difference between the resonant transition frequency and the frequency of the interaction field (i.e. dressing field). This means we can write our Floquet sub-matrix as:

$$
\mathcal{H}_{F}=\mathcal{H}_{Q}+\omega_{p} \mathcal{I}
$$

where

$$
\mathcal{H}_{Q}=\left[\begin{array}{cccc}
0 & 0 & \frac{M_{1}}{2} & \frac{M_{3}}{2} e^{i \phi} \\
0 & 0 & \frac{M_{4}}{2} e^{i \phi} & \frac{M_{2}}{2} \\
\frac{M_{1}}{2} & \frac{M_{4}}{2} e^{-i \phi} & -\delta & 0 \\
\frac{M_{3}}{2} e^{-i \phi} & \frac{M_{2}}{2} & 0 & -\delta
\end{array}\right]
$$

Solving $\mathcal{H}_{Q}$ for the eigenvalues $q_{i}\left(+\omega_{p}\right)$ gives us the energy levels of the states with the interaction field. But first we will calculate the value of $M_{1}, M_{2}, M_{3}, M_{4}$. 


\subsubsection{Calculating the Matrix Elements}

Equation 3.11 defines the time independent, non-zero matrix elements to be:

$$
\begin{aligned}
& M_{1}=E\langle 42 s+1 / 2(t)|z| 41 p+1 / 2(t)\rangle \\
& M_{2}=E\langle 42 s-1 / 2(t)|z| 41 p-1 / 2(t)\rangle \\
& M_{3}=g E\langle 42 s+1 / 2(t)|x| 41 p-1 / 2(t)\rangle \\
& M_{4}=g E\langle 42 s-1 / 2(t)|x| 41 p+1 / 2(t)\rangle
\end{aligned}
$$

where $x$ and $z$ can be defined in terms of Edmonds $\mathrm{C}$ tensors:

$$
\begin{aligned}
& z=r C_{1}^{0} \\
& x=\frac{r}{\sqrt{2}}\left(-C_{1}^{1}+C_{1}^{-1}\right)
\end{aligned}
$$

r is the radial component. $C_{q}^{k}$, the Edmond's $\mathrm{C}$ tensor, defined by:

$$
C_{k}^{q}=\sqrt{\frac{4 \pi}{2 k+1}} Y_{k}^{q}
$$

where $Y_{k}^{q}$ is a spherical harmonic.

To calculate the matrix elements we will use the Wigner-Eckart Theorem [9]:

$$
\begin{aligned}
\left\langle\alpha_{2} j_{2} m_{2}\left|T_{k}^{q}\right| \alpha_{1} j_{1} m_{1}\right\rangle & =\left\langle\alpha_{2} j_{2}|| T_{k}|| \alpha_{1} j_{1}\right\rangle\left\langle j_{2} m_{2} \mid k q, j_{1} m_{1}\right\rangle \\
& =\left\langle\alpha_{2} j_{2}|| T_{k}|| \alpha_{1} j_{1}\right\rangle(-1)^{j_{2}-m_{2}}\left(\begin{array}{ccc}
j_{2} & k & j_{1} \\
-m_{2} & q & m_{1}
\end{array}\right)
\end{aligned}
$$

The Wigner $3-\mathrm{j}$ symbol is a useful tool because for it to be non-zero, $m_{1}+m_{2}+q=0$. This selection rule is a simple way to check that the other potential matrix elements (such as $E q_{e}\langle 42 s \pm 1 / 2|z| 41 p \mp 1 / 2\rangle$ and $\left.E q_{e}\langle 42 s \pm 1 / 2|x g| 41 p \pm 1 / 2\rangle\right)$ are zero. However, barring a few simple cases, Wigner 3-j symbols are complicated to calculate. It's often easiest to look them up in a table [9] or use a program such as Mathematica which has the formulas built in to get a result. In 
our case:

$$
\begin{aligned}
\left(\begin{array}{ccc}
1 / 2 & 1 & 1 / 2 \\
\mp 1 / 2 & 0 & \pm 1 / 2
\end{array}\right) & =\sqrt{\frac{1}{6}} \\
\left(\begin{array}{ccc}
1 / 2 & 1 & 1 / 2 \\
\mp 1 / 2 & \pm 1 & \mp 1 / 2
\end{array}\right) & =-\sqrt{\frac{1}{3}}
\end{aligned}
$$

Now substituting numbers in for our first matrix elements we get:

$$
\begin{aligned}
M_{1} & =E\langle 42 s 1 / 2|z| 41 p 1 / 2\rangle \\
& =E\left\langle 41 p 1 / 2\left|r C_{1}^{0}\right| 42 s 1 / 2\right\rangle \\
& =E R C(-1)^{1 / 2-1 / 2}\left(\begin{array}{ccc}
1 / 2 & 1 & 1 / 2 \\
-1 / 2 & 0 & 1 / 2
\end{array}\right) \\
& =\frac{E R C}{\sqrt{6}}
\end{aligned}
$$

where $\mathrm{R}$ is the radial matrix element $\langle 42 s|r| 41 p\rangle=42^{2} 0.92=1623$ a.u. and $\mathrm{C}$ is the reduced matrix element $\left\langle s 1 / 21 / 2|| C_{1}|| p 1 / 21 / 2\right\rangle$. The other three matrix elements are:

$$
\begin{aligned}
M_{2} & =E\langle 42 s-1 / 2|z| 41 p-1 / 2\rangle \\
& =-\frac{E R C}{\sqrt{6}} \\
M_{3} & =g E\langle 42 s 1 / 2|x| 41 p-1 / 2\rangle \\
& =g E e^{i \phi}\left\langle 41 p 1 / 2\left|\frac{r}{\sqrt{2}}\left(-C_{1}^{1}+C_{1}^{-1}\right)\right| 42 s-1 / 2\right\rangle \\
& =\frac{g E e^{i \phi}}{\sqrt{2}} R(-1) C(-1)^{1 / 2-1 / 2}\left(\begin{array}{ccc}
1 / 2 & 1 & 1 / 2 \\
-1 / 2 & 1 & -1 / 2
\end{array}\right) \\
& =\frac{E R C}{\sqrt{6}} g e^{i \phi}
\end{aligned}
$$




$$
\begin{aligned}
M_{4} & =g E\langle 42 s-1 / 2|x| 41 p 1 / 2\rangle \\
& =\frac{E R C}{\sqrt{6}} g e^{i \phi}
\end{aligned}
$$

There is a common factor in four above matrix elements we will define as:

$$
M=\frac{E R C}{2 \sqrt{6}}
$$

Using the calculated matrix elements, the Hamiltonian is:

$$
\mathcal{H}_{Q}=\left[\begin{array}{cccc}
0 & 0 & M & M g e^{i \phi} \\
0 & 0 & M g e^{i \phi} & -M \\
M & M g e^{-i \phi} & -\delta & 0 \\
M g e^{-i \phi} & -M & 0 & -\delta
\end{array}\right]
$$

In the next subsection we will calculate $C$. Once we have that we will calculate the matrix eigenvalues in Section 3.2.4.

\subsubsection{Calculating the Reduced Matrix Element}

We calculate $\mathrm{C}$ with a relation from Edmonds [9] for a tensor operator $T_{k}$ working on only the first part of a coupled scheme $\left(\gamma j_{1} j_{2} J M\right)$. For our problem, the scheme is $\left(n l s j m_{j}\right)$ where $C_{1}$ acts only on the orbital angular momentum $l$ and not the spin $s$. The general relation is [9]:

$$
\begin{aligned}
& \left\langle\gamma^{\prime} j_{1}^{\prime} j_{2} J^{\prime} \| T_{k}|| \gamma j_{1} j_{2} J\right\rangle \\
& \quad=(-1)^{j_{1}^{\prime}+j_{2}+J+k} \sqrt{[2 J+1]\left[2 J^{\prime}+1\right]}\left\{\begin{array}{ccc}
j_{1}^{\prime} & J^{\prime} & j_{2} \\
J & j_{1} & k
\end{array}\right\} \times\left\langle\gamma^{\prime} j_{1}^{\prime}|| T_{k}|| \gamma j_{1}\right\rangle
\end{aligned}
$$


where $\left\{\begin{array}{ccc}j_{1}^{\prime} & J & j_{2} \\ J & j_{1} & k\end{array}\right\}$ is a 6 -j symbol and the new reduced matrix element can be defined by [9]:

$$
\left\langle\gamma^{\prime} l^{\prime}|| C_{k}|| \gamma l\right\rangle=(-1)^{l^{\prime}} \sqrt{[2 l+1]\left[2 l^{\prime}+1\right]}\left\{\begin{array}{ccc}
l^{\prime} & k & l \\
0 & 0 & 0
\end{array}\right\}
$$

Calculating equation 3.35 for our specific case gives:

$$
\begin{aligned}
C & =\left\langle s 1 / 21 / 2|| C_{1}|| p 1 / 21 / 2\right\rangle \\
& \left.=(-1)^{0+1 / 2+1 / 2+1 \sqrt{[21 / 2+1][21 / 2+1]}} \begin{array}{ccc}
0 & 1 / 2 & 1 / 2 \\
1 / 2 & 1 & 1
\end{array}\right\} \times\left\langle s|| C_{1}|| p\right\rangle \\
& =12 \frac{1}{\sqrt{6}}(-1)^{0} \sqrt{[20+1][21+1]}\left\{\begin{array}{lll}
0 & 1 & 1 \\
0 & 0 & 0
\end{array}\right\} \\
C & =-\frac{2}{3}
\end{aligned}
$$

\subsubsection{Calculating the Hamiltonian's Eigenvalues}

As stated in equation 3.32, the Hamiltonian is:

$$
\mathcal{H}_{Q}=\left[\begin{array}{cccc}
0 & 0 & M & M g e^{i \phi} \\
0 & 0 & M g e^{i \phi} & -M \\
M & M g e^{-i \phi} & -\delta & 0 \\
M g e^{-i \phi} & -M & 0 & -\delta
\end{array}\right]
$$

where $M=E R C / 2 \sqrt{6}$. We have found $C=2 / 3$ and $R=1623$ a.u. This gives us:

$$
M=\frac{E R C}{2 \sqrt{6}}=\frac{E 1623}{2 \sqrt{6}} \frac{2}{3}=220.8 E
$$

where $E$ is the magnitude of the electric field in the $\hat{z}$ direction in atomic units.

It is now possible to calculate the eigenvalues of Hamiltonian. These are the energy levels of the eigenstates in the presence of a dressing field. They are summarized in Table 3.1. These eigenvalues depend only on the characteristics of the dressing field $\vec{E}_{d}(t)=E(\hat{z} \cos (\omega t)+\hat{x} g \cos (\omega t+\phi))$. 


\begin{tabular}{|c|c|c|c|}
\hline Case & Defined by & Eigenvalues $q_{i}$ & Deg. \\
\hline 1 & $g=0, \delta \neq 0$ & $\frac{1}{2}\left(-\delta \pm \sqrt{4 M^{2}+\delta^{2}}\right)$ & 2 \\
\hline 2 & $g \neq 0, \delta \neq 0$ & $\frac{1}{2}\left(-\delta \pm \sqrt{4 M^{2}+\delta^{2}+4 M^{2} g^{2}-4 M^{2} \sqrt{-\left(g e^{i \phi}-g e^{-i \phi}\right)^{2}}}\right)$ & 1 \\
& & $\frac{1}{2}\left(-\delta \pm \sqrt{4 M^{2}+\delta^{2}+4 M^{2} g^{2}+4 M^{2} \sqrt{-\left(g e^{i \phi}-g e^{-i \phi}\right)^{2}}}\right)$ & \\
\hline 3 & $g=0, \delta=0$ & $\pm M$ & 2 \\
\hline 4 & $g \neq 0, \delta=0$ & $\pm \sqrt{M^{2}+M^{2} g^{2}-M^{2} \sqrt{-\left(g e^{i \phi}-g e^{-i \phi}\right)^{2}}}$ & 1 \\
& & $\pm \sqrt{M^{2}+M^{2} g^{2}+M^{2} \sqrt{-\left(g e^{i \phi}-g e^{-i \phi}\right)^{2}}}$ & \\
\hline
\end{tabular}

Table 3.1: Summary of Eigenvalues and degeneracies for different cases.

There are four cases described in Table 3.1:

1. linearly polarized field that is off resonance.

2. elliptically polarized field that is off resonance.

3. linearly polarized field that is on resonance.

4. elliptically polarized field that is on resonance.

Figure 3.2 graphs cases 1 and 2 from Table 3.1 showing the evolution of the eigenstates based on $\delta$, the difference between the field frequency and the transition resonant frequency. When $\delta$ is large, the eigenstates are $\omega_{p}$ and $\omega_{s}-\omega$. As $\delta \rightarrow 0$, the eigenstates become a mixture of the four states creating an avoided crossing. Figure $3.2 \mathrm{~b}$ shows a sample of case 2 where there are 4 separable eigenstates. $M=110.6 E$ is set equal to 1 for the purposes of the graphs.

The on resonance, linearly polarized case (case 3) is particularly simple. The eigenvalues are $\pm 110.6 E$ with a degeneracy of two. This means for a linear dressing field there will be two peaks when the probe frequency is scanned over the transition. The splitting between those peaks is proportional to the dressing field strength. The eigenstates for this case are also the simplest. If we define the vectors as $[|42 s,+1 / 2\rangle,|42 s,-1 / 2\rangle,|41 p,+1 / 2\rangle,|41 p,-1 / 2\rangle]$, the eigenstates are $[0,1,0,1]$, $[-1,0,1,0],[0,-1,0,1]$ and $[1,0,1,0]$.

The eigenvalues for the elliptically polarized, on resonance case (case 4) can be graphed as a function of $g$ for different $\phi$. The general form is recorded in Table 3.1. Figure 3.3 shows the positive eigenvalues. The exact negative is reflected below the axis $\left(\omega_{p}=0\right)$. From this figure 


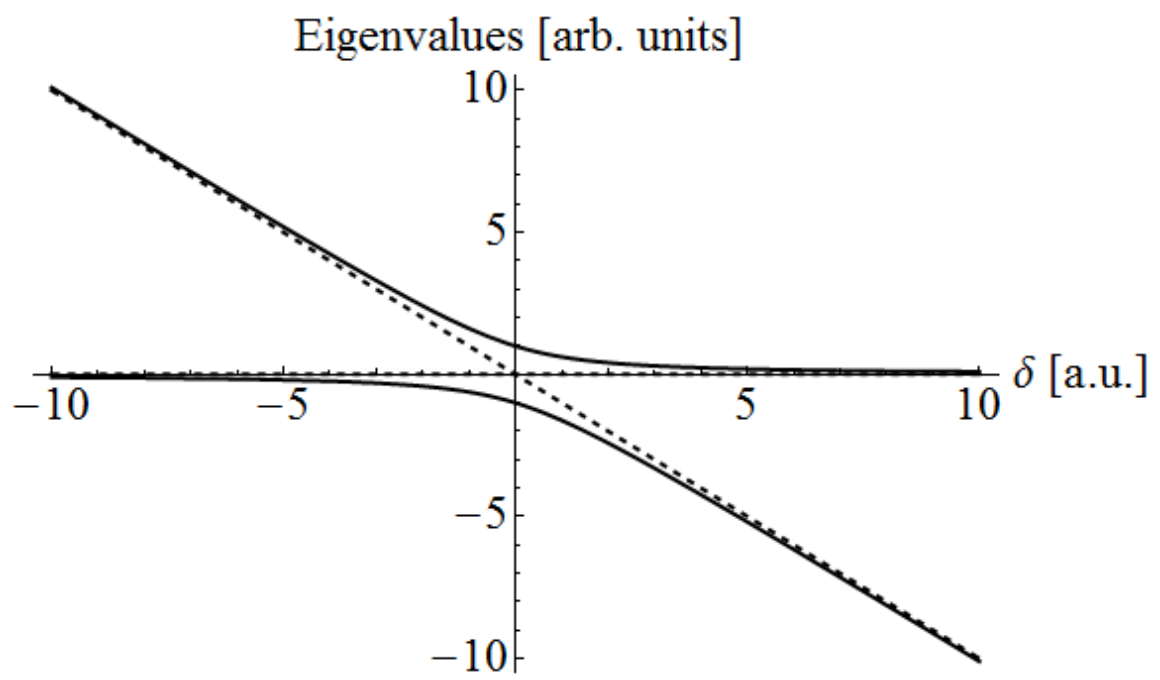

(a) Define $M=1$ and $g=0$ - This defines a linearly polarized interaction field.

Eigenvalues [arb. units]

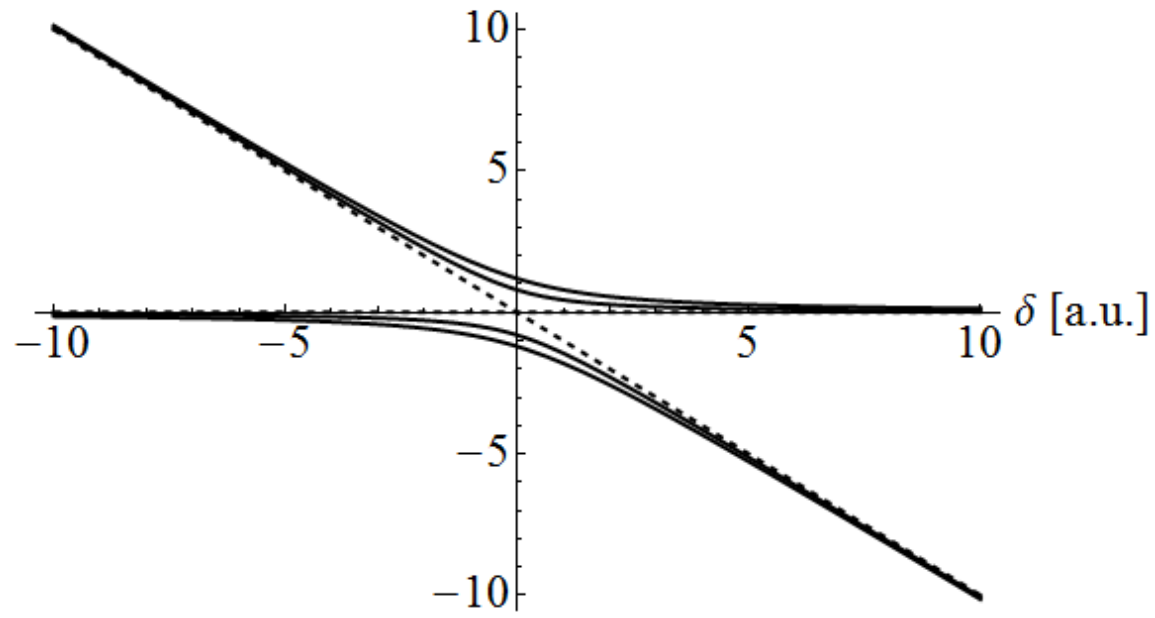

(b) Define $M=1$ and $g e^{i \phi}=.2 e^{i 5 \pi / 12}$ - This defines an elliptically polarized interaction field similar to those seen experimentally.

Figure 3.2: Graphs of the off resonance eigenvalues as a function of $\delta \propto \omega$. The dashed lines represent the eigenvalues if there was no interaction Hamiltonian. 
several conclusions can be drawn as to the nature of the splitting. Variations in the field over the volume of the MOT cause broadening in the lineshape. If there is a greater variation in $\phi$ compared to $g$ over the MOT volume, the inner peak will be wider. Greater variation over $g$ causes the outer peak to be wider. We can calculate the eigenstates for this case for $\phi=\pi / 2$. If we define the vectors as $[|42 s,+1 / 2\rangle,|42 s,-1 / 2\rangle,|41 p,+1 / 2\rangle,|41 p,-1 / 2\rangle]$, the eigenstates are $[-i, 1, i, 1],[-i,-1,-i, 1],[i, 1,-i, 1]$, and $[i,-1, i, 1]$.

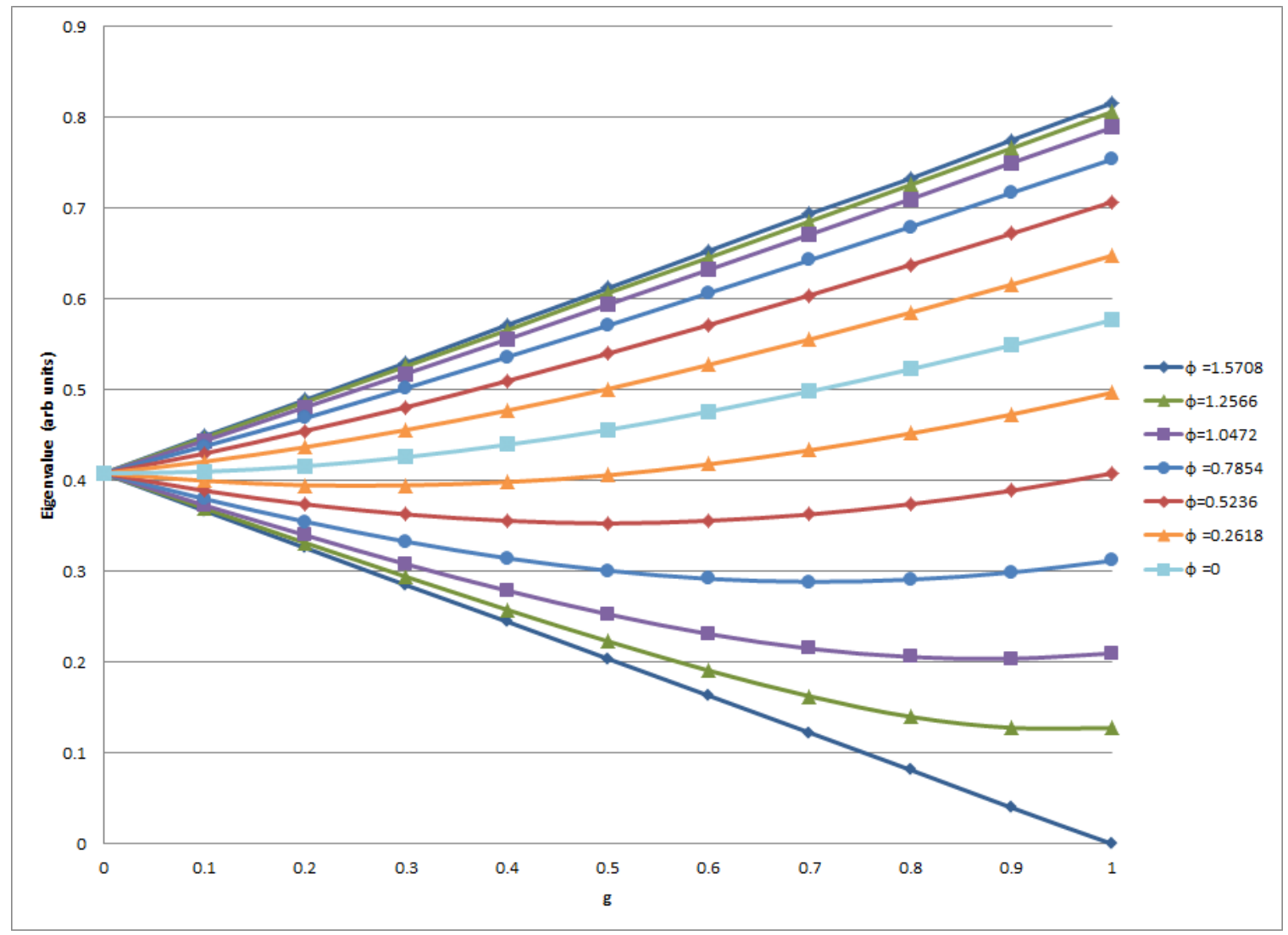

Figure 3.3: Graph of positive eigenvalues as a function of $\mathrm{g}$. This is in arbitrary units and sets $M=1$. 


\subsection{Experimental Approach}

The first step to any experiment on this set up is to create a successful Magneto-Optical Trap (MOT) (see Section 2.1). Next, a portion of the atoms trapped in the MOT are excited to Rydberg states using a pulsed blue laser (see Section 2.2). Directly after the pulsed laser excited the atoms, two microwave pulses are emitted from microwave horns in the direction of the MOT. One of the pulses is a probe field, scanned over the $41 s_{1 / 2} \rightarrow 41 p_{1 / 2}$ transition. The second field is the dressing field pulse, set at the resonance between $41 p_{1 / 2}$ and $42 s_{1 / 2}$. This is shown in Figure 3.4. The second field is 200ns wider and centered in time on the probe pulse. Both pulses can be emitted in either vertical or horizontal polarization by changing the physical orientation of the microwave horn through addition of a $90^{\circ}$ twist waveguide. The probe pulse is optimized to maximize signal but avoid power broadening. The microwaves have to be heavily attenuated to stay in this regime. The dressing pulse amplitude will be varied to get the desired splitting.

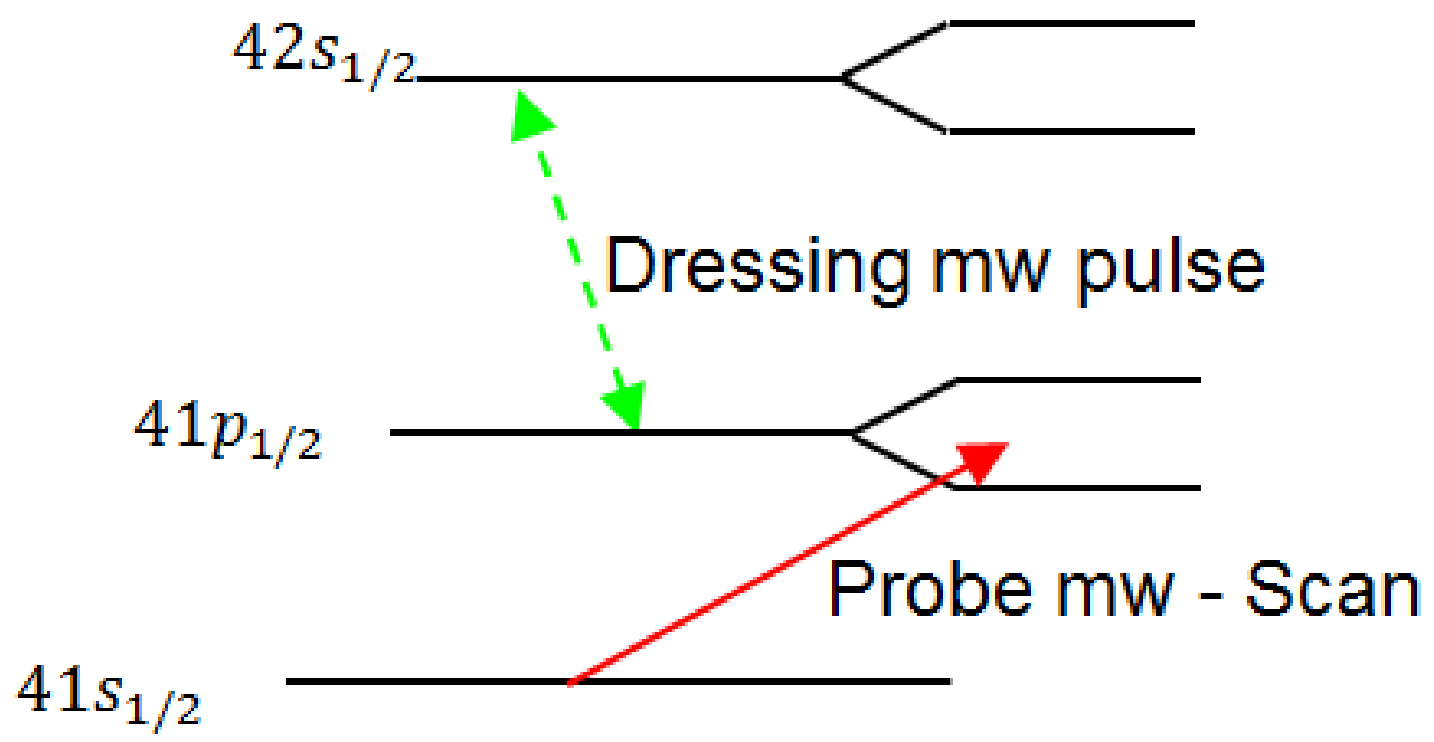

Figure 3.4: Transitions used in the Dressed State Experiment

After the microwave pulses, the atoms are ionized by a pulse applied to the field ionization rods. The electrons or ions are detected with a micro-channel plate detector (MCP) (see Section 2.2.2). 
Figure 3.5 shows the timing diagram for this experiment. The MCP sends the signal to a gated integrator. A gate is placed over the 41p signal and it is integrated and sent to the computer. The computer program controls the probe frequency. The probe frequency is scanned over the $41 \mathrm{~s}-42 \mathrm{p}$ transition. The graph of 41p signal vs probe frequency (microwave scan) is the basis for all data taken in this experiment. When there is a dressed state, the gated $41 \mathrm{p}$ signal is actually $41 \mathrm{p}+42 \mathrm{~s}$ since the atoms oscillate between the $41 \mathrm{p}$ and $42 \mathrm{~s}$ states and they can not be separated on the oscilloscope. The microwave scans are then fit with multiple Lorentzian curves in the program Origin to determine center frequencies and widths of the peaks.

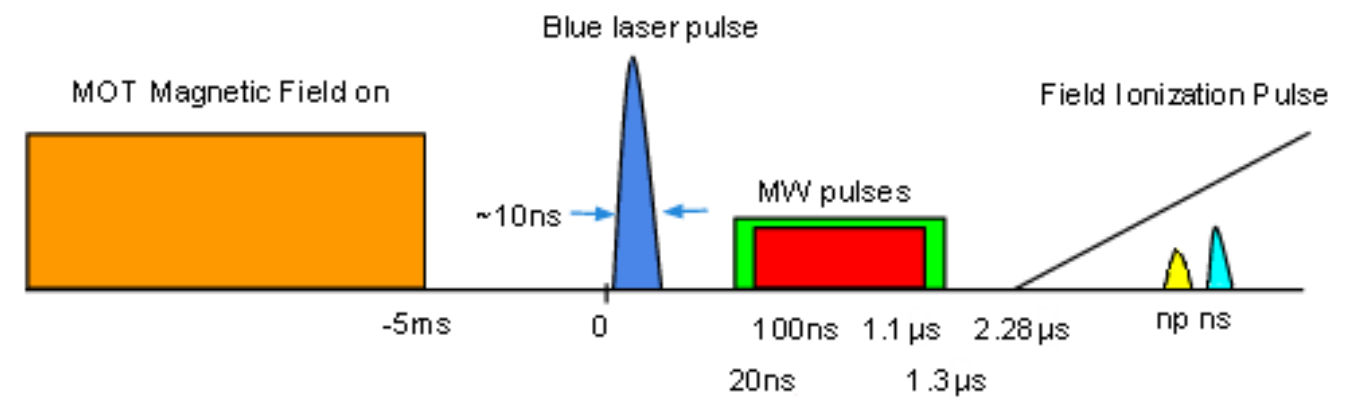

Figure 3.5: Timing diagram for the Dressed State Experiment

The experimental approach is similar to the one described in Section 2.5, but with a dressing field created using a second microwave field tuned to the $41 \mathrm{p}-42 \mathrm{~s}$ transition. In fact, it is necessary to first get a transform-limited, narrow linewidth microwave transition without the dressing field before adding it. This experiment uses all the equipment described in more detail in Chapter 2 .

\subsection{Results and Analysis}

In this section various characteristics of the dressed state transition are reported and analyzed. This includes the effect of changing the dressing power, the dressing frequency, and both probe and dressing polarizations. We also calculate characteristics of the dressing field from the microwave scans. 


\subsubsection{Polarization of the Fields}

The polarization of the field is far more important than was expected before preforming this experiment. Since the initial microwave (MW) fields are linearly polarized, it was expected that the field at the MOT would be linearly polarized. In that case, there would only be two peaks on the microwave scan and likewise would be only two eigenvalues for the Hamiltonian matrix. While sometimes that was the case, it frequently was not - especially in the regimes of high dressing field amplitude.

The microwave fields that leave the microwave horns are linearly polarized either vertically or horizontally depending on the horn orientation. However, the field that reaches the MOT is not necessarily linear in polarization. Section 3.2 derives that while a linearly polarized dressing field generates two eigenvalues each with a degeneracy of two, elliptical polarization breaks that degeneracy and causes a secondary splitting. This secondary splitting is on a smaller scale than

the initial dressed state splitting. For this reason higher dressing field amplitudes are needed to fully separate the peaks. If the elliptical component of the polarization is weak enough that it's not possible to define separate peaks, it can still cause broadening or small side lobes.

Figure 3.6 shows the effect of changing the dressing field orientation. There is considerably more secondary splitting for a vertically polarized dressing field than a horizontally polarized dressing field. To try to understand why that is, we modeled the microwave scattering from the field ionization rods. This model is developed in Chapter 4.

The vertically polarized field (defined as $\hat{z}$ ) is parallel to the field ionization rods; this causes it to scatter a great deal. However, if the direction of propagation of the field (defined by $\vec{k}$ ) is perfectly perpendicular to the rods (in the $\hat{y}$ direction), the field scatters only in the $\hat{z}$ polarization. If $\vec{k}$ has a $k_{z}$ component, then there is scattering in the $\hat{x}$ and $\hat{y}$ direction. While the magnitude of the scattered fields is greater for vertically polarized fields, the amount of elliptical polarization (defined by g) was found to be comparable to horizontally polarized light $(g \sim 0.1)$. There must be additional sources of scattering such as the walls of the vacuum chamber, but that is harder to model. Horizontally polarized light $(\hat{x})$ always scattered into both $\hat{x}$ and $\hat{y}$ polarizations. The summation of the scattering from all 4 rods creates an interference pattern over the volume of the MOT that contains elliptically polarized light. Table 4.3 in Section 4.5 lists $[g, \phi]$ for different initial conditions and different positions in the MOT. 


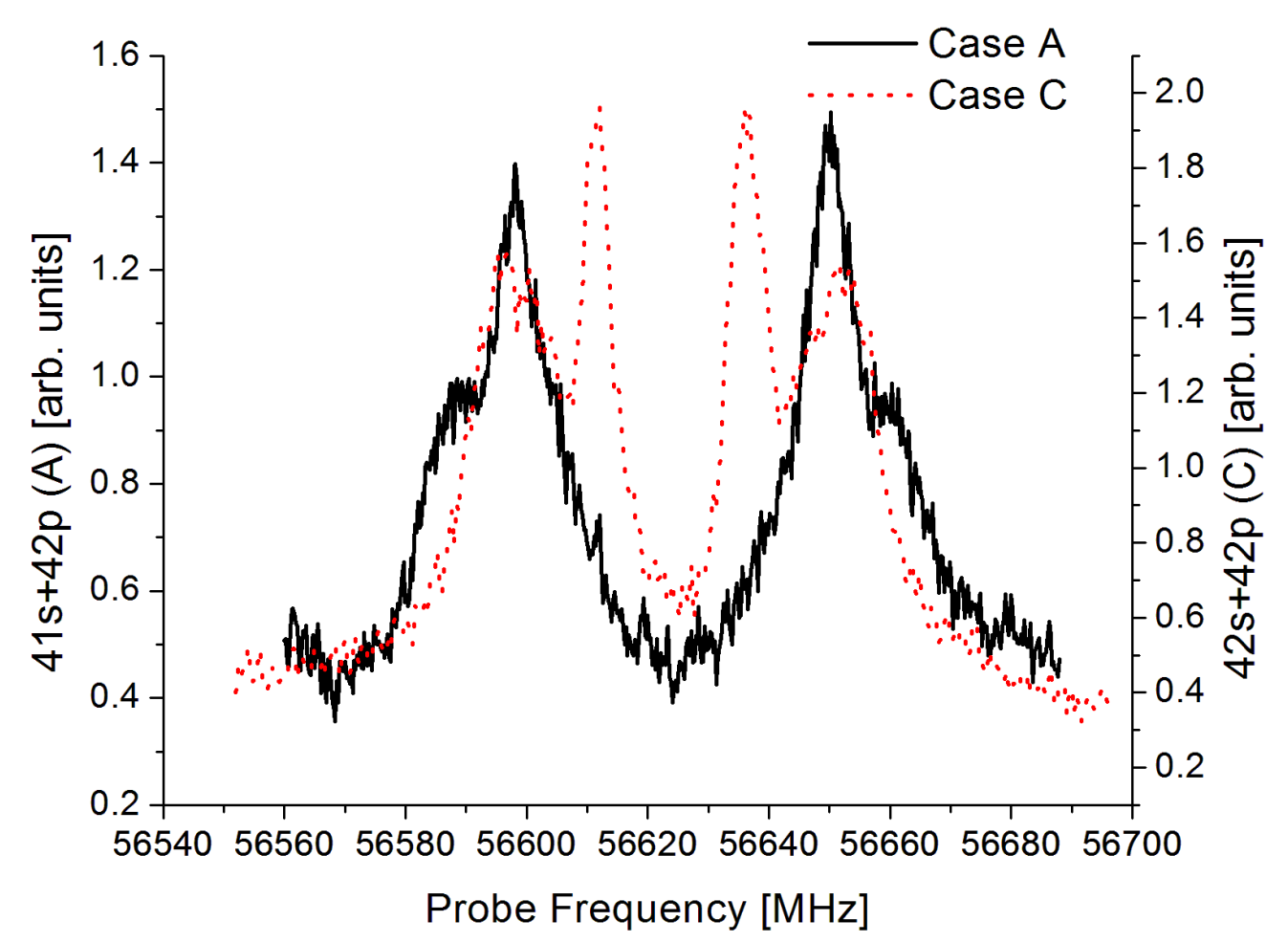

Figure 3.6: Graph comparing horizontal dressing polarization (Case A) with vertical dressing polarization (Case C). The probe field polarization is kept horizontal. There is a drastic change in the resonance lineshape. 


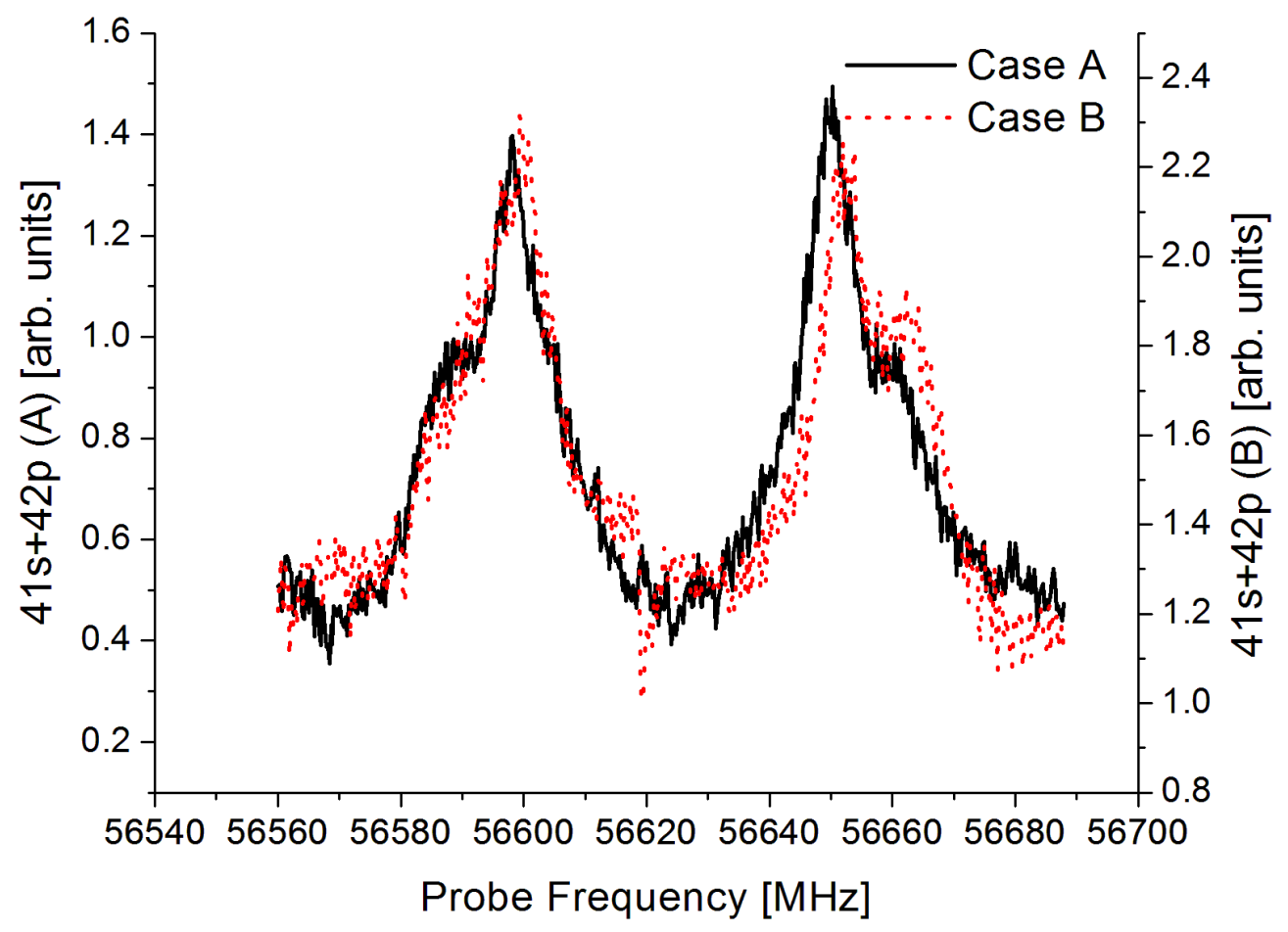

Figure 3.7: Graph comparing horizontal probe polarization (Case A) with vertical probe polarization (Case B). The dressing field polarization is kept horizontal. There is little change in the resonance lineshape. 
Figure 3.7 shows changing the probe field orientation; it has negligible change on the lineshape, whether or not there is discernible secondary splitting. The probe field couples the $41 s_{1 / 2}$ states with the $41 p_{1 / 2}$ part of the dressed state eigenvectors. There is no electric dipole $41 s_{1 / 2} \rightarrow 42 s_{1 / 2}$ coupling. Based on the microwave scattering theory discussed in the previous paragraph, the probe field experiences scattering and likely is elliptically polarized and varied over the volume of the MOT. However, looking at the experimental data, that doesn't seem to matter. We have been unable to determine a clear theoretical reason why the probe polarization doesn't affect the lineshape.

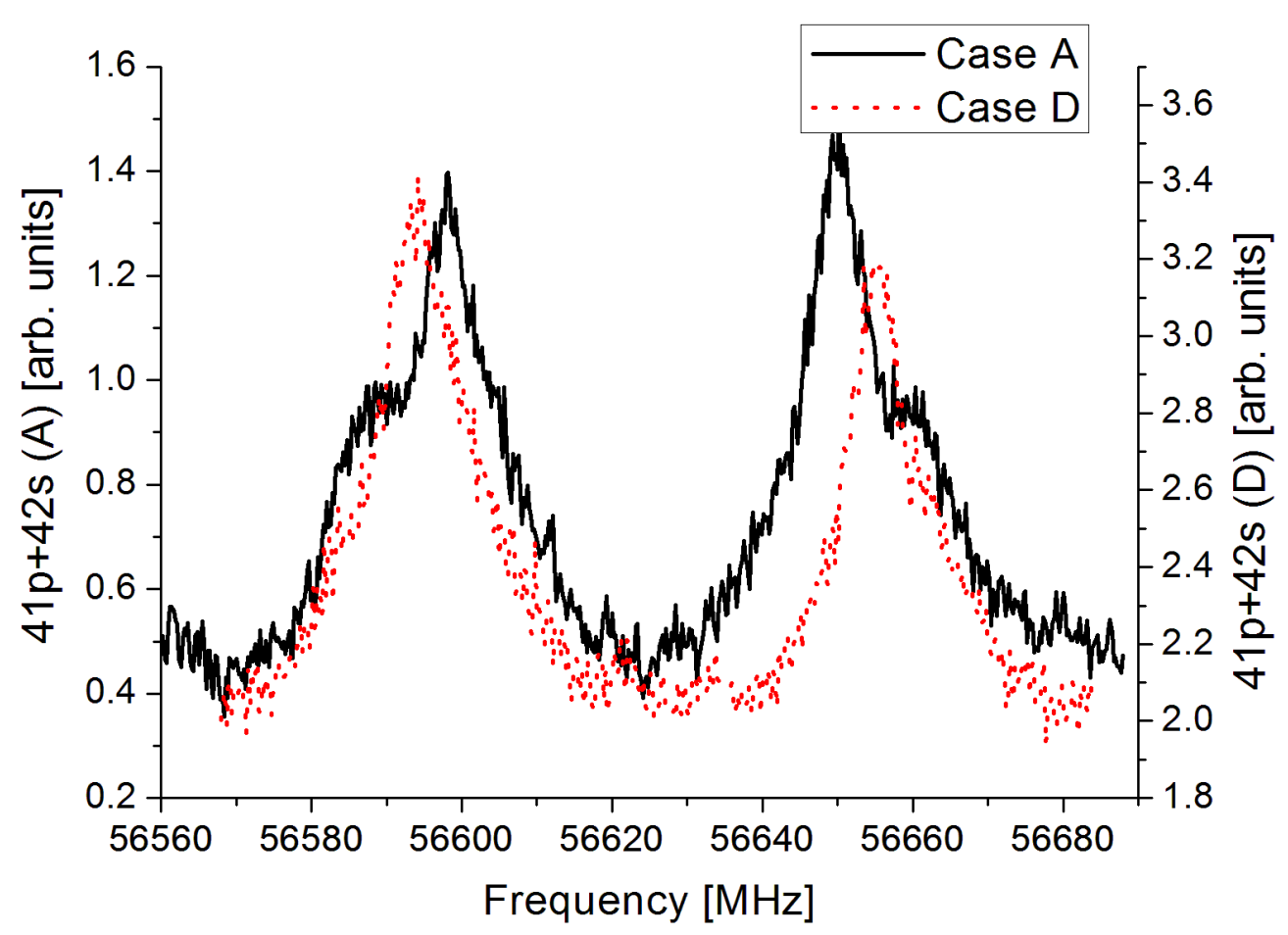

Figure 3.8: Graph comparing two scans both with horizontal dressing and probe polarizations taken four hours apart. There is a small change in the resonance lineshape.

Figure 3.8 shows an example of the change in the lineshape that can occur over the course of a day of experimentation. On the same day before and after taking the scans shown in Section 3.4.3 the same iteration of polarizations in Figures 3.6 and 3.7 were repeated with different amounts of secondary splitting. This can be seen by comparing case A and case D in Figure 3.8. Both have horizontal dressing and probe polarizations. The purpose of showing both cases is to demonstrate 
the different lineshapes (corresponding to different elliptical polarization in the dressing microwave field over the volume of the MOT) that can occur with small changes in the horn position and orientation that result from changing field attenuation and horn orientation. The result of this is that it's difficult (except by trial and error) to get the same field at the MOT.

\subsubsection{Position Dependence}

In an attempt to characterize the scattering pattern, the dressing microwave field horn (and attached waveguides, attenuators, and passive tripler) was shifted parallel to the face of the vacuum chamber. The normal position used for all other measurements in this thesis is the dressing horn centered directly perpendicular to the MOT. Figure 3.9 shows the normal position with an arrow pointing in the direction the horn was shifted, while keeping the orientation the same.

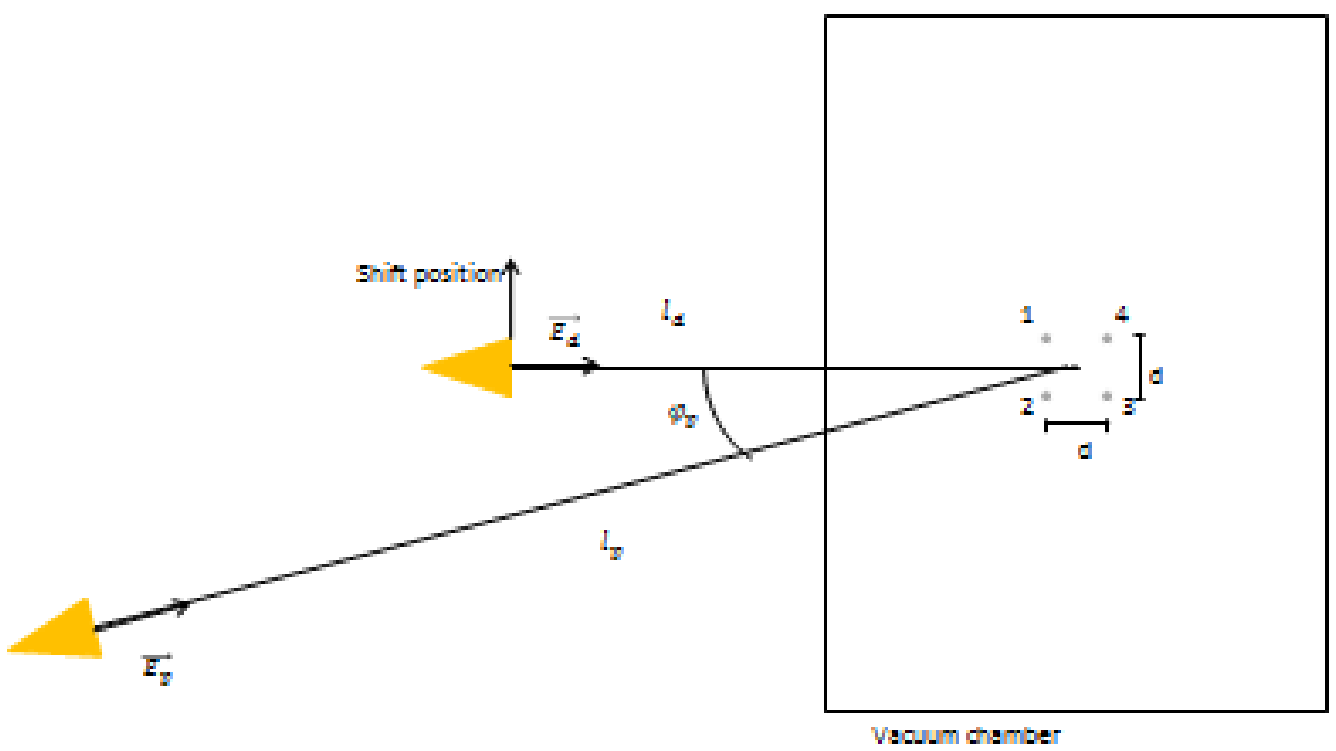

Figure 3.9: Diagram showing the normal orientation of microwave horns with an arrow pointing in the direction it was shifted for the different scans in 3.10 and 3.11

Figures 3.10 and 3.11 show microwave scans for position shifts up to $1.5 \mathrm{~cm}$ with the dressing field polarization horizontal and vertical, respectively. This greatly changes the splitting.

There seems to be a pattern leading to similarly sized peaks in the vertically polarized case and 


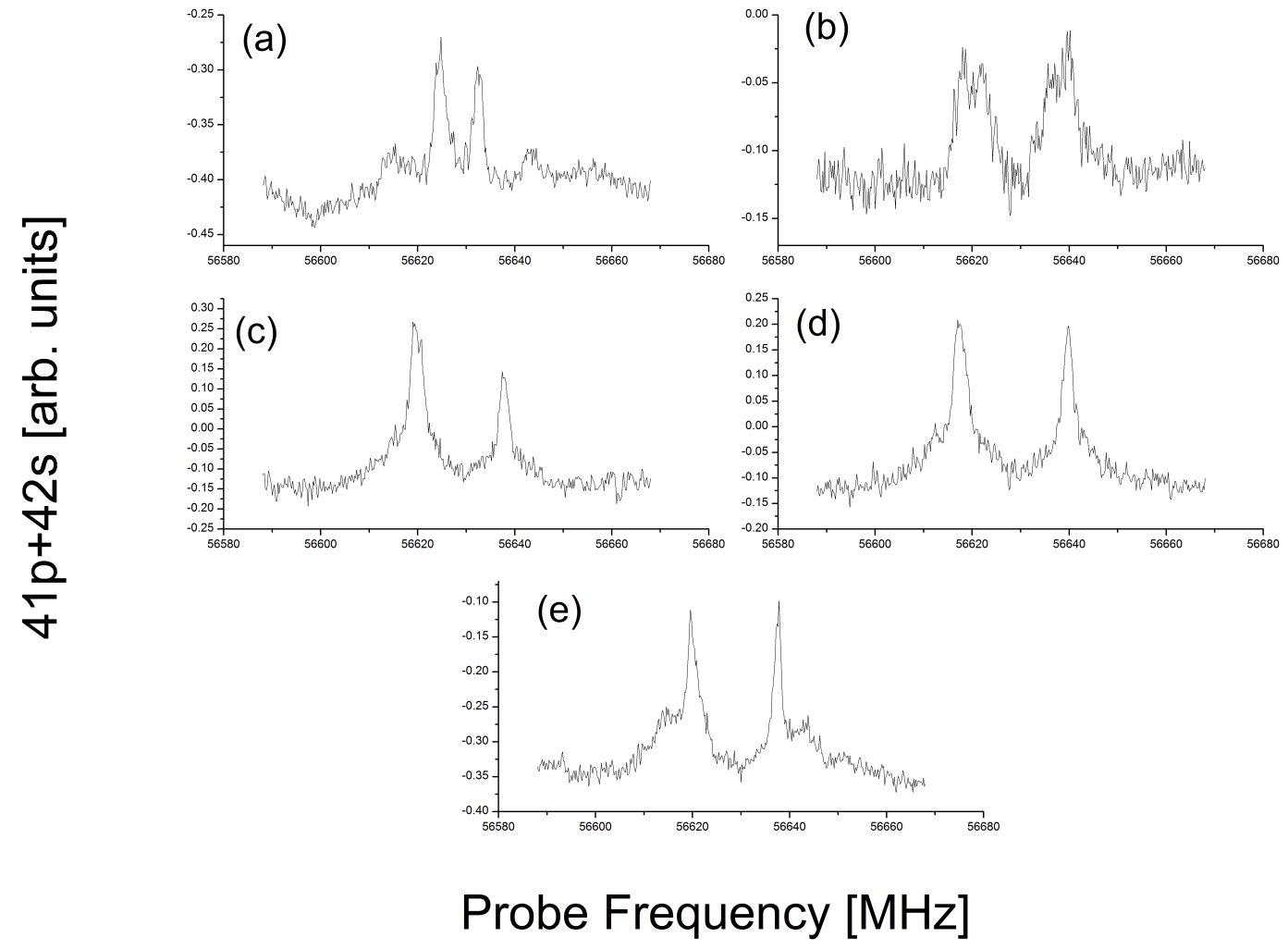

Figure 3.10: The dressing and probe field polarization is horizontal. The position of the dressing horn is shifted by (a) $1.5 \mathrm{~cm}$ (b) $1 \mathrm{~cm}$ (c) $0.5 \mathrm{~cm}$ (d) $0 \mathrm{~cm}$ (e) $-1 \mathrm{~cm}$ relative to normal. 


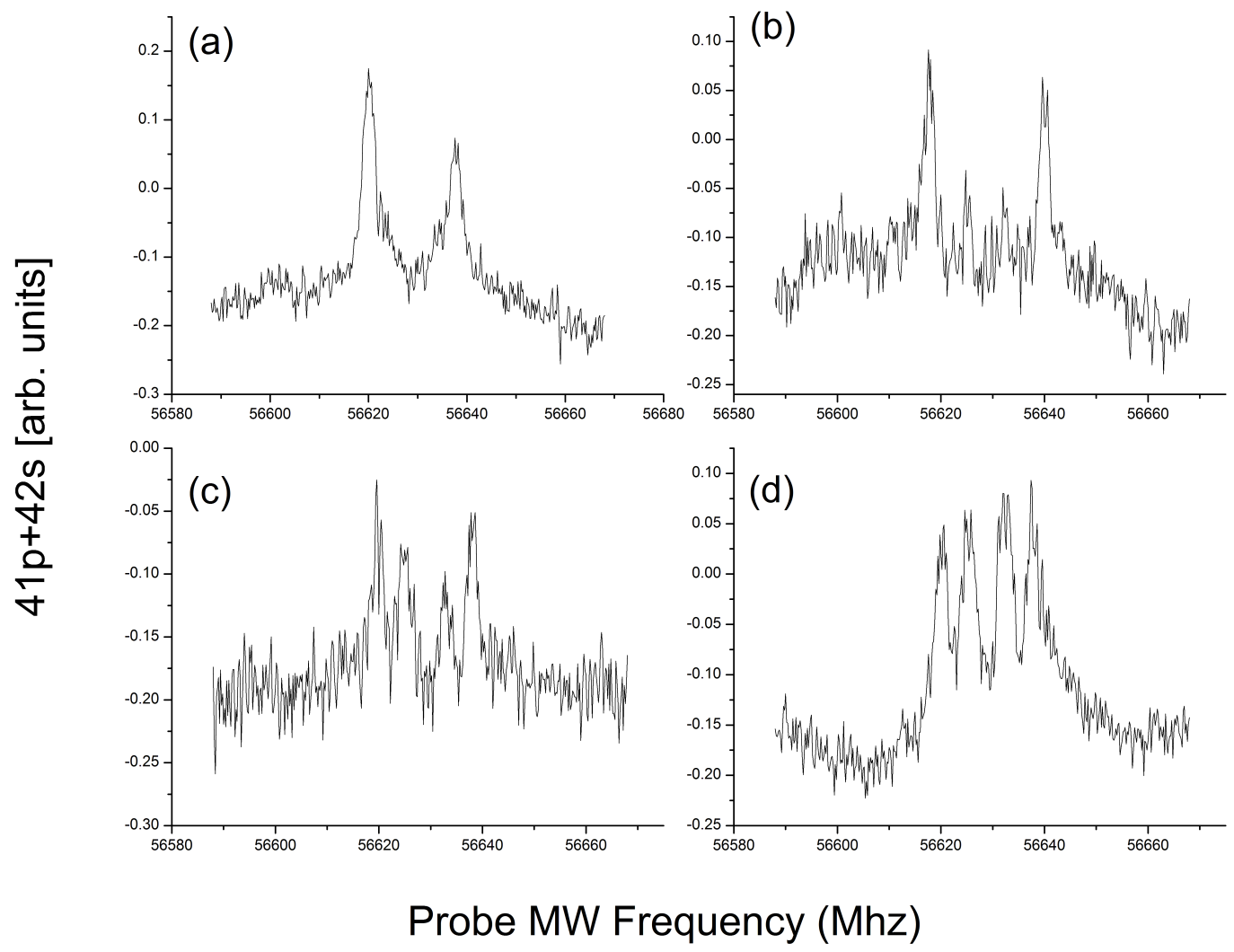

Figure 3.11: The dressing field polarization is vertical and the probe field polarization is horizontal. The position of the dressing horn is shifted by (a) $1.5 \mathrm{~cm}(\mathrm{~b}) 1 \mathrm{~cm}$ (c) $0.5 \mathrm{~cm}$ (d) $0 \mathrm{~cm}$ relative to normal. 
2 peaks in the horizontally polarized case. However, considering the effects from shifts on the scale of millimeters, it's not possible to drawn any strong conclusions in this section.

\subsubsection{Dressing Field Amplitude Dependence}

According to the theory developed in 3.2, the splitting from the dressed states should be proportional to the dressing field amplitude. In the experiment, the MW field amplitude is controlled using precision variable attenuators before the microwave horn. The relative field amplitude can be found as a function of the $\mathrm{dB}$ of attenuation using Equation 3.38.

$$
|E| \propto \sqrt{|P|} \propto \sqrt{10^{-\beta / 10}}
$$

where $|E|=$ magnitude of electric field in arbitrary units, $|P|=$ magnitude of the intensity in arbitrary units, and $\beta=$ attenuation in $\mathrm{dB}$. For our purposes of comparing this to the theory, relative field amplitude is sufficient. Section 3.4.6 discusses using the microwave scan splitting to estimate the dressing field at the MOT.

With no dressing field present, the microwave transition has a transform-limited lineshape. In this experiment a $1 \mu s$ pulse was used, so with zero dressing field the transition width is approximately $1 \mathrm{MHz}$. This can only be seen provided all other sources of broadening (such as microwave power broadening and magnetic field broadening) are minimized. To counter broadening due to external magnetic fields, nulling coils are used and their currents are set as described in Section 2.5. To prevent microwave power broadening of the spectrum, the probe field power is lowered with variable attenuators until the peak is at its transform-limited linewidth.

Next the dressing field was applied and the attenuation on it slowly decreased, the result of which increases the dressing field amplitude logarithmically. Scans of the probe frequency were taken at different dressing field attenuations over the full range of the variable attenuator. Select scans are graphed in Figure 3.12a and 3.13a. Section 3.4.1 discusses the different results obtained from using vertically or horizontally polarized dressing fields, so this experiment was repeated for both cases.

Figures $3.12 \mathrm{c}$ and $3.13 \mathrm{c}$ show that splitting of the field is linearly dependent on the field strength, as is expected from our calculations in Section 3.2. However, for a vertical dressing field polarization, there is a secondary splitting with larger field strengths. According to the theory, this means there 
is enough elliptical polarization that we can see the splitting. However, there may be a smaller third set of peaks on the outside of the larger two. Whether this comes from different elliptical fields (polarization and/or amplitude) in different parts of the MOT or a different effect is uncertain. There may be small secondary peaks for the horizontal polarization at large dressing field strengths, however it's small enough that it can't be distinguished clearly. More analysis on the effect of polarization can be found in Section 3.4.1.

\subsubsection{Detuning dressed frequency}

The frequency of the dressing field is controlled by a microwave synthesizer. It sends a pulsed field to a passive tripler, which sends it through waveguides and attenuators to be emitted by the horn. To observe the effects of a non-resonant dressing frequency, the synthesizer frequency was changed in sets of $5 / 3 \mathrm{MHz}$ (so the emitted frequency changed in steps of $5 \mathrm{MHz}$ ). This was done for $\pm 60 \mathrm{MHz}$. Figure 3.14a shows the microwave scans where the dressing frequency is detuned in steps of $20 \mathrm{MHz}$. The dressing field was close to linear polarization; some secondary splitting can be seen, but all analysis was done fitting to two peaks.

When the frequency is detuned, theoretically we get the pattern shown in Figure 3.2a (shown again in Figure 3.15) for the eigenvalues. In the experiment, we detuned the dressing frequency in steps of $10 \mathrm{MHz}$ and graphed the center frequency of the peaks as a function of the dressing frequency in Figure 3.14b. The dressing frequency in atomic units is $\omega=\omega_{0}+\delta$ and the center frequency of the peaks is proportional to the eigenvalues calculated. The experiment and theory match closely.

\subsubsection{Density Dependence}

The original goal of this experiment was exploring the relationship between the density of Rydberg atoms and linewidth of the dressed and undressed peak, but unfortunately concerns with the polarization ended up taking over. However, some preliminary plots were made and are shown in this section.

Increased density allows for greater atom-atom interaction. The dressed state eigenstates are a combination of $|42 s, \pm 1 / 2\rangle$ and $|41 p, \pm 1 / 2\rangle$. This creates a dipole. The dipole-dipole interaction between atoms should broaden the dressed signal peaks faster than the interactions between the 


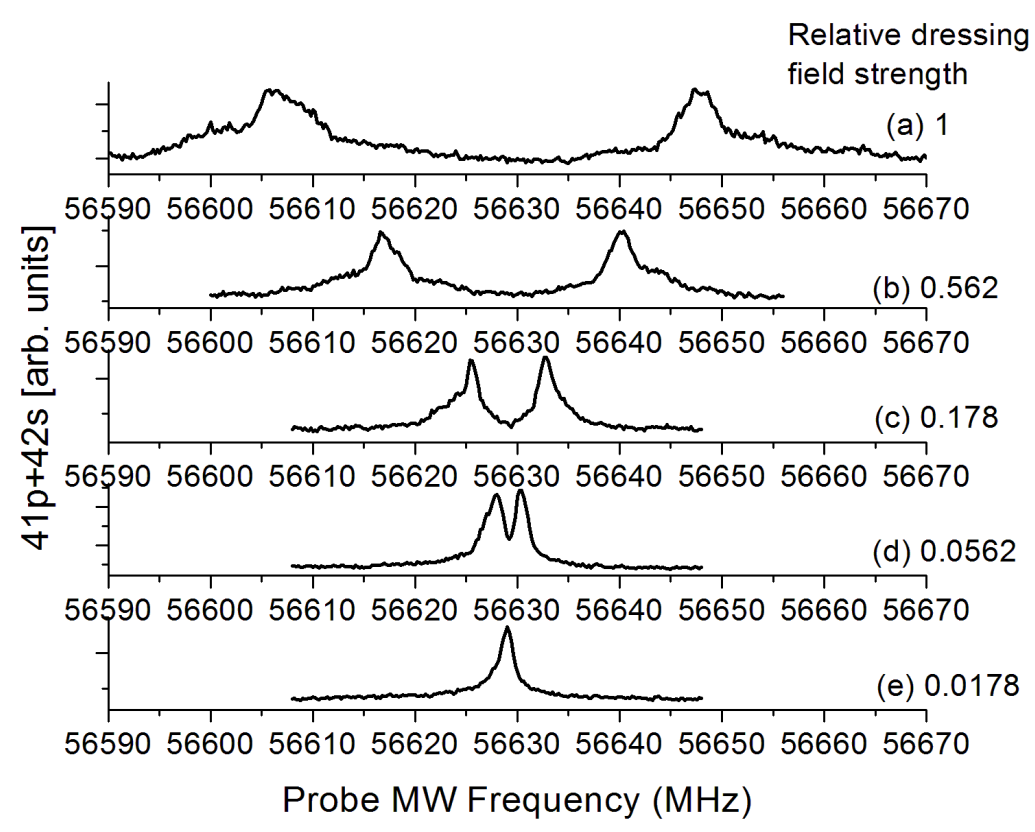

(a) Scans at different dressing field strength showing how they look as it splits

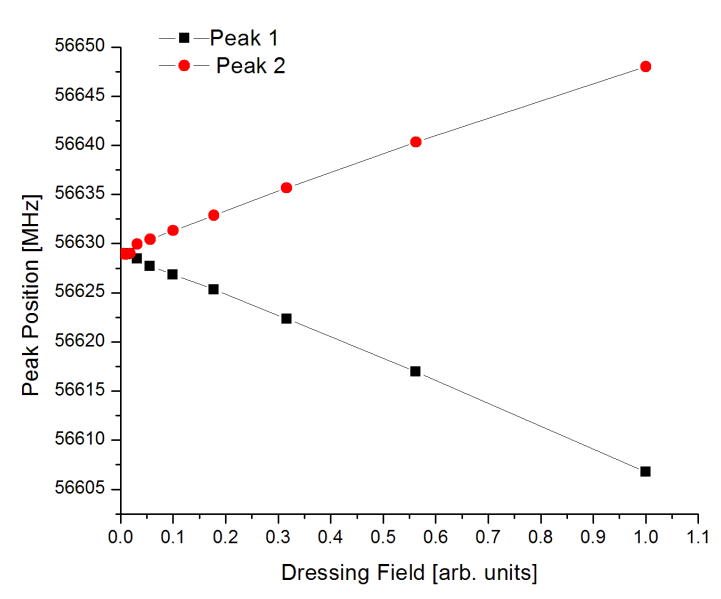

(b) Graph of the center frequencies of the peaks at different dressing field strengths

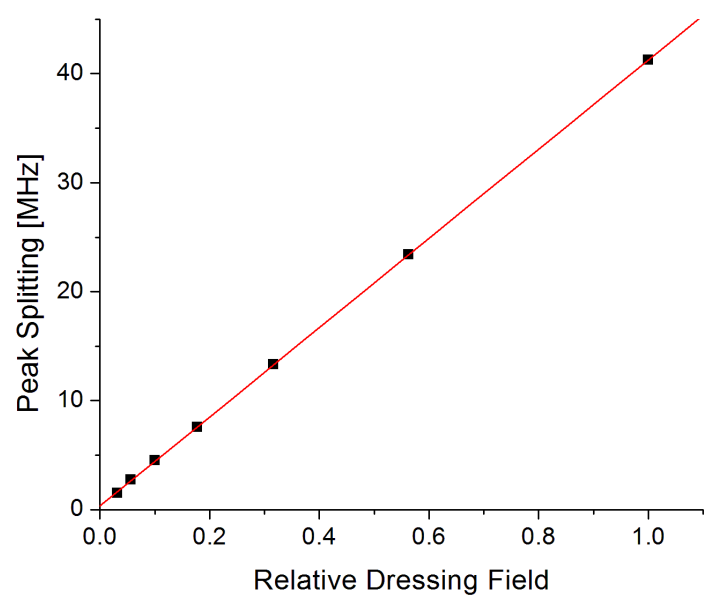

(c) The splitting between the two peaks, judged against a linear fit

Figure 3.12: Scanning over different field strengths, dressing field and probe field with horizontal polarization. 


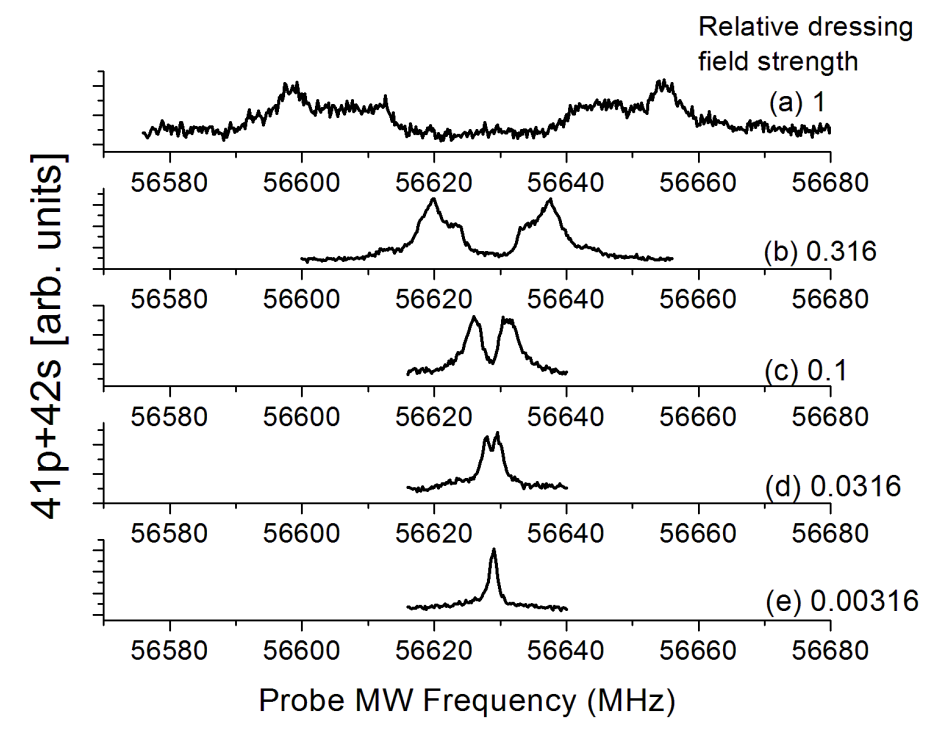

(a) Scans at different dressing field strength showing how they look as it splits

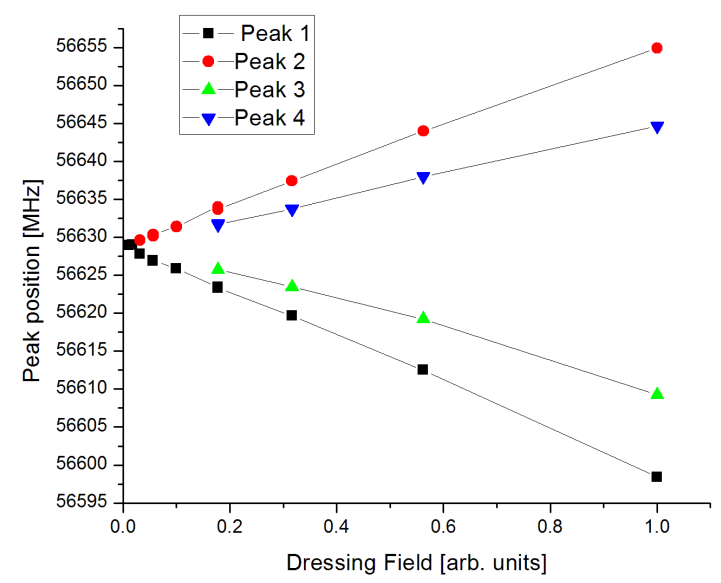

(b) Graph of the center frequencies of the peaks at different dressing field strengths

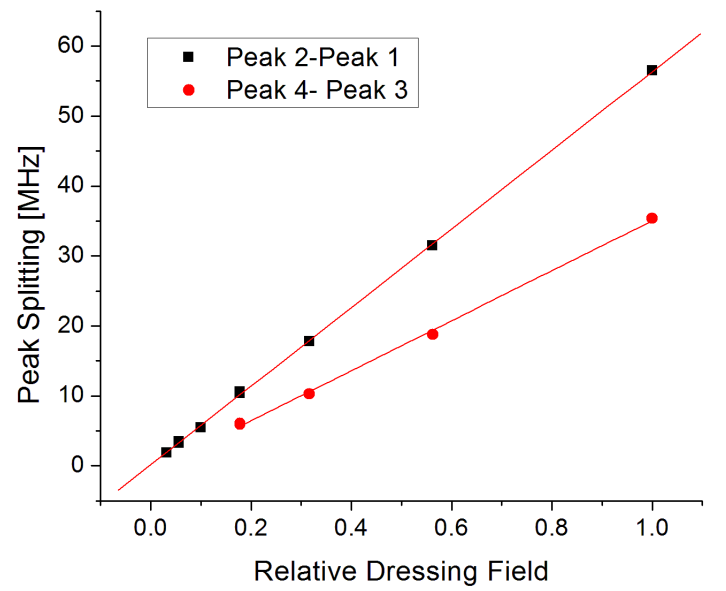

(c) The splitting between the two sets of peaks, judged against a linear fit

Figure 3.13: Scanning over different field strengths, dressing field with vertical polarization and probe field with horizontal polarization. 


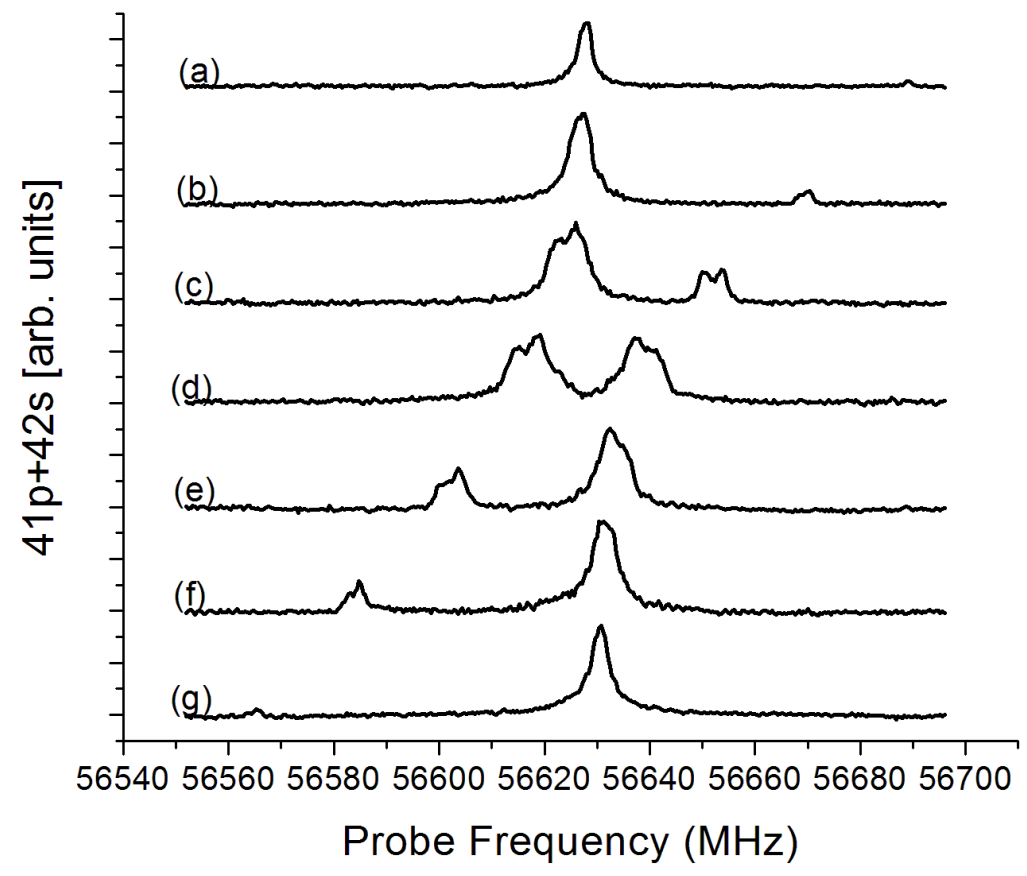

(a) Tuning the dressing frequency in $20 \mathrm{MHz}$ steps: (a) $\omega=\omega_{0}+2 \pi 60 \mathrm{MHz}$ and (g) $\omega=\omega_{0}-2 \pi 60 \mathrm{MHz}$

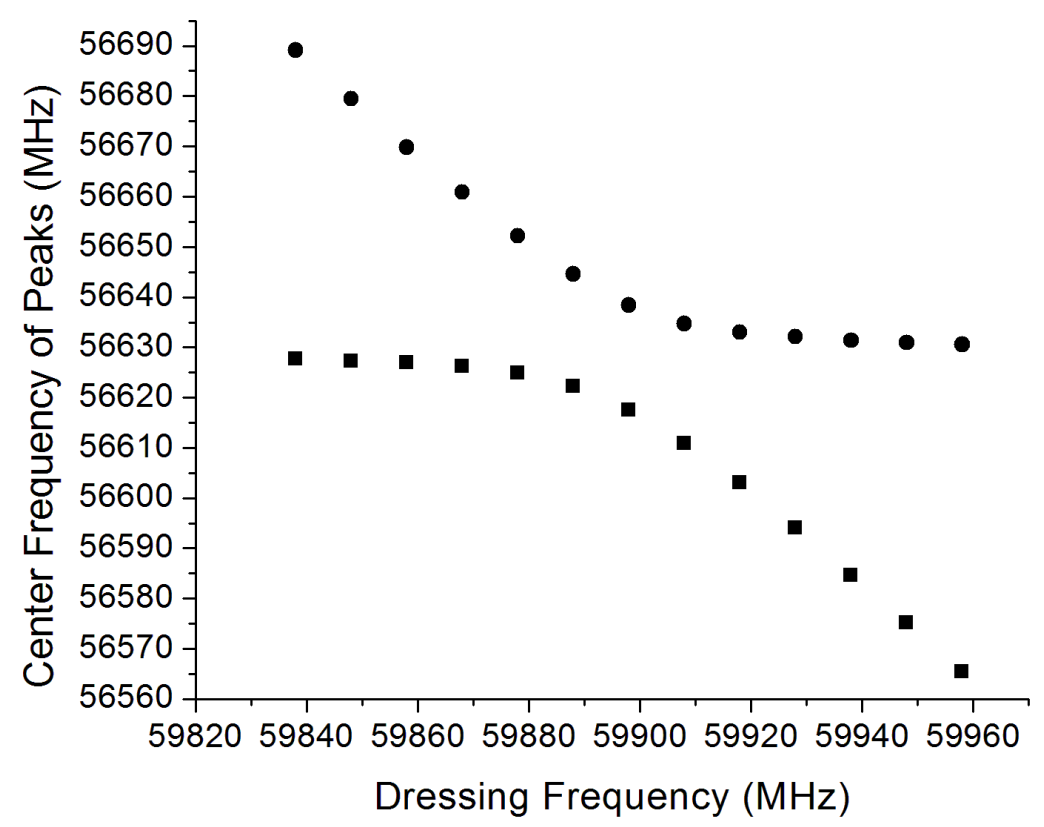

(b) Peak position as a function of dressed frequency shown -the avoid crossing is clear.

Figure 3.14: Scanning the dressing field frequency. 


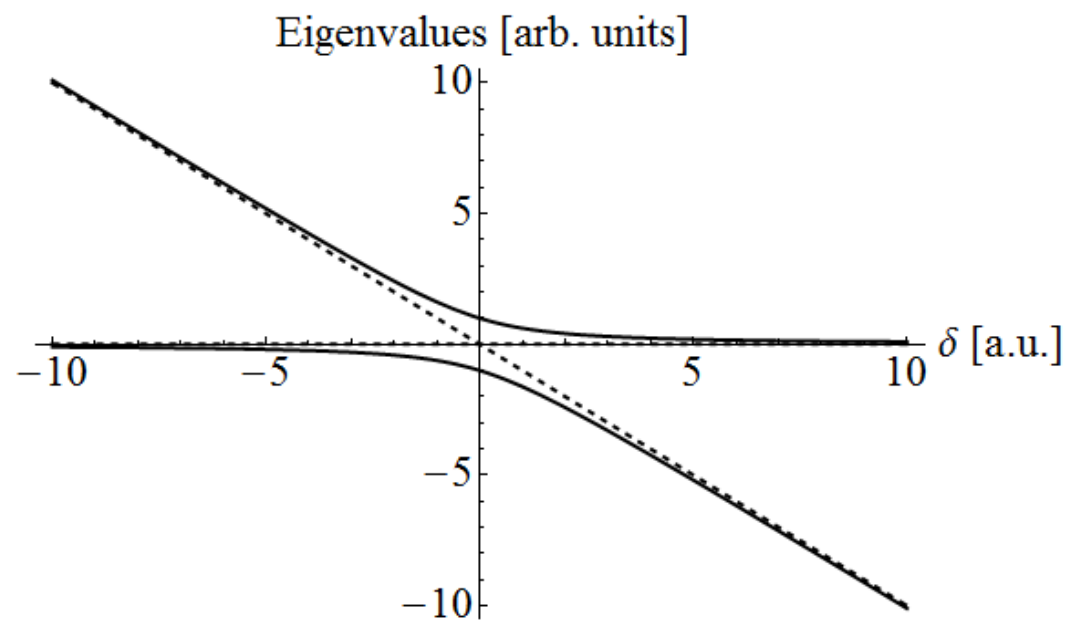

Figure 3.15: Graph of the off resonance eigenvalues as a function of $\delta=\omega-\omega_{0}$ for a linearly polarized interaction field. The dashed lines represent the eigenvalues if there was no interaction Hamiltonian.

undressed atoms. Figure 3.16 shows dressed and undressed states at low and high density. To study this interaction further, a higher density MOT needs to be obtained.

The density of Rydberg atoms during an experiment is primarily controlled by the intensity of the blue laser (which excites the Rydberg atoms) through the MOT. However, the amount of atoms that can be excited is limited by the number of atoms in the MOT. Too high a blue laser intensity can deplete the atoms trapped in the MOT (for Rydberg atoms are no longer trapped once they are excited and field ionized) faster than it can replenish itself during the time the MOT magnetic field is on in between blue laser shots. One solution to this is to change the pulse frequency of the YAG (and by extension the the frequency of the experiment cycle) from $30 \mathrm{~Hz}$ to $15 \mathrm{~Hz}$. A different possible solution is to increase the number of atoms in the MOT. This can be changed primarily through three factors: trapping and repump laser alignment, trapping laser power, or getter current.

The laser alignment effects the cross section of the trap. Perfectly aligned lasers that all overlap exactly in the center of the vacuum chamber (and therefore the center of the MOT magnetic field) create a smaller, denser trap. This is something that has to be adjusted daily.

Trapping laser power should be increased for this setup to get a higher density MOT. At the current laser power level, the trap has a linear dependence between laser field amplitude and number of atoms in the MOT. Ideally, the laser power would reach a saturation point, where small increases or decreases in the laser power wouldn't effect the number of atoms in the MOT. Greater laser 


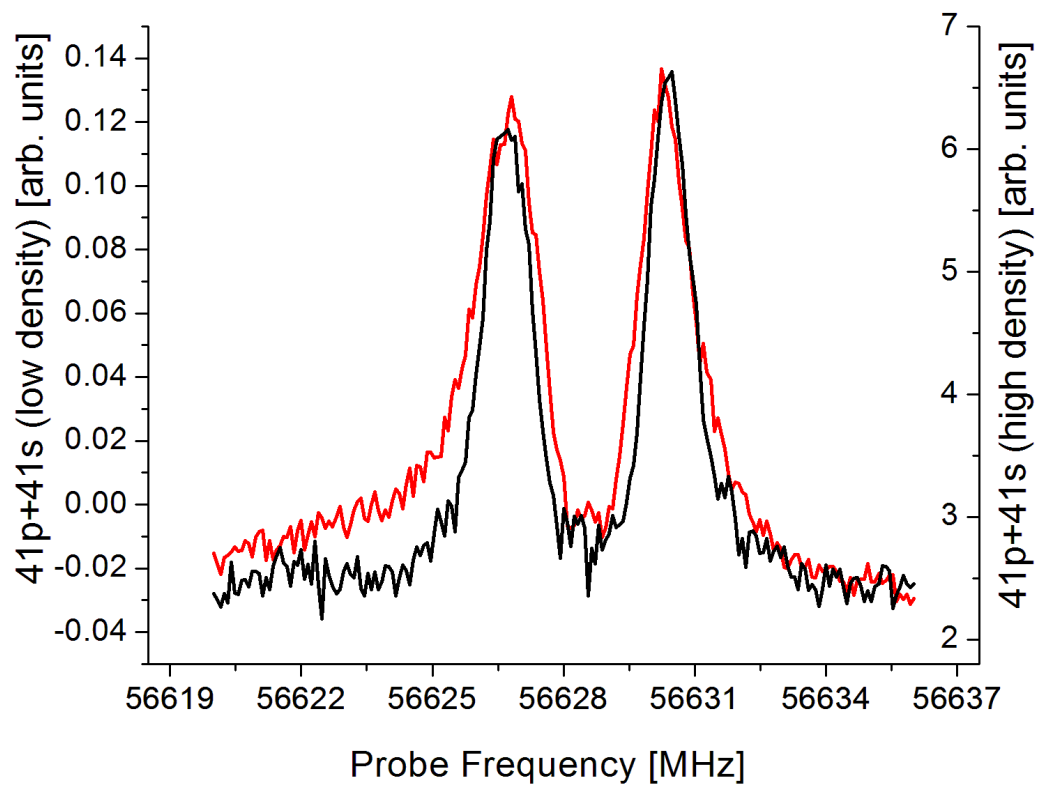

(a) Shows dipole-dipole broadening in dressed states.

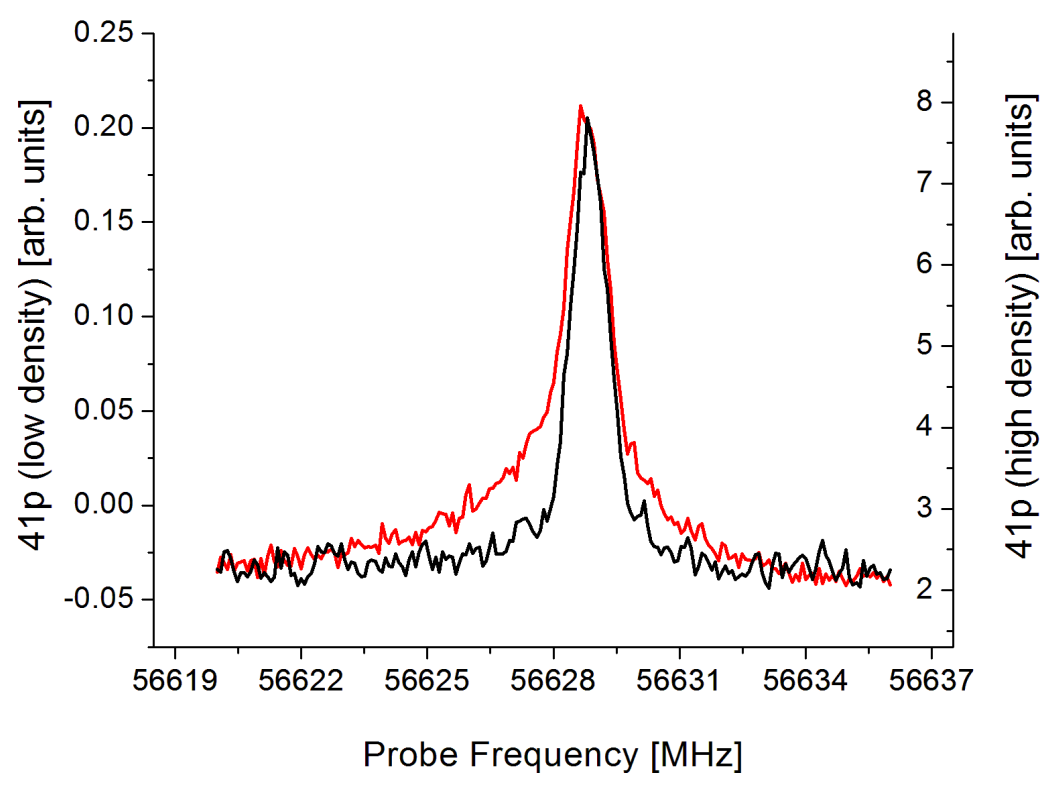

(b) Shows density broadening in undressed state.

Figure 3.16: Low and high density MW scans. Probe and dressing field polarizations are both horizontal. 
power could be obtained though a higher power laser diode, potentially a new laser diode of the same model (if this one is suffering from decreased power output due to age), or use of a master-slave laser setup. A master-slave laser setup allows the full power of the slave laser to go to the MOT, instead of deflecting some to the saturated absorption locking setup. See Hyunwook Park's thesis

[2] for more details, as that is the type of setup used in his experiment. The simplest option is use a higher power laser diode.

The getter current controls the number of rubidium atoms in the vacuum chamber. The getter current "boils off" atoms from the getter. As the getter ages and has fewer atoms, higher getter currents are necessary to have the same number of atoms in the vacuum chamber. Since atoms are continuously lost due to the ion pump (which maintains the high vacuum) and field ionization, the number of atoms in the chamber that could be trapped at any given time is the equilibrium between the different rates. However, it's very important not to have too high of a getter current and too many atoms in the chamber. Too many atoms will lead to a large number of atoms outside the trap contributing to background noise and creating additional collisions and interactions. Careful exploration of the getter current compared to MOT amplitude, MOT rise time, and vacuum pressure would probably prove useful to further exploration of high density states. See Hyunwook Park's thesis [2] for more details, as he preforms this comparison for his setup.

\subsubsection{Relating Peak Frequency to Dressing Field Magnitude and Polarization}

The microwave scans created through scanning the probe field frequency over a range centered on $\omega_{p}$ contain 1, 2, or 4 peaks that represent an equivalent number of eigenvalues at the probe frequencies. The peaks are not a lines but approximately Lorentzian lineshapes for multiple reasons. Some reasons broaden the width of the atomic resonances and are controlled for as discussed in Section 2.5. There is also broadening because the field is inhomogeneous over the volume of the MOT. The frequencies where there is a signal correspond to an atomic resonance which correspond to the eigenvalues derived in Section 3.2. The center of the peaks corresponds to the "average" eigenvalue for the MOT. It can be found though fitting the microwave scans with multiple Lorentzian curves - one for each peak. If we convert the center frequency from $\mathrm{Hz}$ to a.u. we can equate that to the 
eigenvalues. The conversion for frequency between $\mathrm{MHz}$ and atomic units (a.u.) is [10]:

$$
1 \text { a.u. }=6.5796 * 10^{9} \mathrm{MHz}
$$

Once in atomic units we can equate the frequency of the transitions to the energy resonance because they are related by a factor of $\hbar=1$ a.u. The eigenvalues are written in terms of the field it's possible to solve for the dressing field as a function of the eigenvalues. The number calculated relates to the dressing field seen by the majority of the atoms. There is a limit of separability for the eigenvalues which is the limit of the fitting algorithm to fit overlapping curves as separate peaks.

The on resonance eigenvalues calculated in Section 3.2.4 for the dressed state Hamiltonian for linearly polarized fields are:

$$
\begin{aligned}
& \lambda_{1}^{(l i n)}=+M+\omega_{p} \\
& \lambda_{2}^{(l i n)}=-M+\omega_{p}
\end{aligned}
$$

and for elliptically polarized fields are:

$$
\begin{aligned}
& \lambda_{1}^{(e l l)}=+\sqrt{M^{2}+M^{2} g^{2}+M^{2} \sqrt{-\left(g e^{i \phi}-g e^{-i \phi}\right)^{2}}}+\omega_{p} \\
& \lambda_{2}^{(e l l)}=+\sqrt{M^{2}+M^{2} g^{2}-M^{2} \sqrt{-\left(g e^{i \phi}-g e^{-i \phi}\right)^{2}}}+\omega_{p} \\
& \lambda_{3}^{(e l l)}=-\sqrt{M^{2}+M^{2} g^{2}+M^{2} \sqrt{-\left(g e^{i \phi}-g e^{-i \phi}\right)^{2}}}+\omega_{p} \\
& \lambda_{4}^{(e l l)}=-\sqrt{M^{2}+M^{2} g^{2}-M^{2} \sqrt{-\left(g e^{i \phi}-g e^{-i \phi}\right)^{2}}}+\omega_{p}
\end{aligned}
$$

where the dressing field is defined by $\vec{E}_{d}(t)=E(\hat{z} \cos (\omega t)+\hat{x} g \cos (\omega t+\phi))$ and $M=220.8 E$ in atomic units. The center frequency of the scan is $\omega_{p}=56.6286 \mathrm{GHz}=8.06067 * 10^{-6}$ a.u., the energy of the undressed $41 p_{1 / 2}$ state.

By converting the peak center frequencies into atomic units and equating them with the eigenvalues for either linearly polarized or elliptically polarized fields it is possible to estimate the average magnitude of the field and it's polarization. For linearly polarized fields it is simple, however for elliptically polarized fields we essentially only have two independent eigenvalues $\left(\lambda_{1}^{(e l l)}, \lambda_{2}^{(e l l)}\right)$ and we have three field variables $(E, g, \phi)$. 
The simplest way to solve this is by setting $\phi=\pi / 2$. This assumption is supported by the fact that the modeled field scattered from the field ionization rods (Chapter 4) was found to have $\phi=\pi / 2$ for all cases. However, it is known that there are other non-negligible sources of scattering (such as the walls of the vacuum chamber) that have not been modeled and it's uncertain if $\phi=\pi / 2$ holds true for them as well.

Setting $\phi=\pi / 2$ simplifies the eigenvalues to:

$$
\begin{gathered}
e^{i \pi / 2}-e^{-i \pi / 2}=2 i \\
\lambda_{1}^{(e l l)}=+M \sqrt{1+g^{2}+2 g}+\omega_{p} \\
=+M(1+g)+\omega_{p} \\
\lambda_{2}^{(e l l)}=+M(1-g)+\omega_{p} \\
\lambda_{3}^{(e l l)}=-M(1+g)+\omega_{p} \\
\lambda_{4}^{(e l l)}=-M(1-g)+\omega_{p}
\end{gathered}
$$

Since $\lambda_{1}$ and $\lambda_{3}$ are the same splitting from $\omega_{p}$ (as are $\lambda_{2}$ and $\lambda_{4}$ ), when comparing theory with experiment it makes sense to average the experimental distance between the inner peaks and the center frequency and between the outer peaks and the center frequency. We will define these "average" eigenvalues as:

$$
\begin{aligned}
& \lambda_{1}=\frac{\left(\lambda_{1}^{(e l l)}-\lambda_{3}^{(e l l)}\right)}{2}=M(1+g) \\
& \lambda_{2}=\frac{\lambda_{2}^{(e l l)}-\lambda_{4}^{(e l l)}}{2}=M(1-g)
\end{aligned}
$$

where $\lambda_{1}$ and $\lambda_{2}$ are the average splitting of the outer and inner experimental peaks from the center frequency $\omega_{p}$.

Solving for $M=220.8 E$ and $g$ gives:

$$
\begin{gathered}
M=\frac{\lambda_{1}+\lambda_{2}}{2} \\
E=\frac{\lambda_{1}+\lambda_{2}}{441.6} \\
g=\frac{\lambda_{1}-\lambda_{2}}{\lambda_{1}+\lambda_{2}}
\end{gathered}
$$


where $\lambda_{1} \geq \lambda_{2}, \phi=\pi / 2$ and the field is in atomic units.

To convert the field from atomic units to $\mathrm{V} / \mathrm{cm}$, we use the following conversion [10]:

1 a.u. $=4.1341 * 10^{9} \mathrm{~V} / \mathrm{cm}$

We can calculate magnitude of the field $E_{d}$ and the power $P$ corresponding to the dressing field with:

$$
\begin{aligned}
E_{d} & =\sqrt{E^{2}+(E g)^{2}}=E \sqrt{1+g^{2}}(\mathrm{~V} / \mathrm{cm}) \\
P & =\frac{V^{2}}{R}=\frac{E_{d}^{2}}{Z_{0}}(\mathrm{~W} / \mathrm{cm})^{2}
\end{aligned}
$$

Where the resistance $R=Z_{0}=377 \Omega$ is the impedance of free space and the potential $V=E_{d}$ is the dressing field magnitude.

\begin{tabular}{|c|c|c|c|c|c|}
\hline Vertical Dressing Pol. & $3.11 \mathrm{~d}$ & $3.11 \mathrm{c}$ & & $3.6 \mathrm{C}$ & \\
\hline Splitting $(\mathrm{MHz})\left(\lambda_{1}\right)$ & 9.230 & 8.795 & 8.915 & 27.795 & 24.090 \\
\hline Splitting $(\mathrm{MHz})\left(\lambda_{2}\right)$ & 3.900 & 3.470 & 5.130 & 12.280 & 10.760 \\
\hline $\mathrm{g}$ & 0.406 & 0.434 & 0.269 & 0.387 & 0.382 \\
\hline $\mathrm{E}(\mathrm{mV} / \mathrm{cm})$ & 18.7 & 17.5 & 20.0 & 57.0 & 49.6 \\
\hline$E_{d}(\mathrm{mV} / \mathrm{cm})$ & 20.2 & 19.0 & 20.7 & 61.1 & 53.1 \\
\hline Power $\left(\mu \mathrm{W} / \mathrm{cm}^{2}\right)$ & 1.078 & 0.960 & 1.136 & 9.917 & 7.476 \\
\hline
\end{tabular}

Table 3.2: Horizontal polarization, experimental dressing field

\begin{tabular}{|c|c|c|c|c|c|}
\hline Horizontal Dressing Pol. & $3.10 \mathrm{c}$ & $3.10 \mathrm{c}$ & & $3.6 \mathrm{~A}$ & $3.7 \mathrm{~B}$ \\
\hline Splitting $(\mathrm{MHz})\left(\lambda_{1}\right)$ & 9.045 & 12.795 & 16.075 & 37.600 & 34.005 \\
\hline Splitting $(\mathrm{MHz})\left(\lambda_{2}\right)$ & 9.045 & 11.115 & 11.515 & 25.525 & 25.885 \\
\hline $\mathrm{g}$ & 0.000 & 0.070 & 0.165 & 0.191 & 0.136 \\
\hline $\mathrm{E}(\mathrm{mV} / \mathrm{cm})$ & 25.739 & 34.020 & 39.256 & 89.816 & 85.213 \\
\hline$E_{d}(\mathrm{mV} / \mathrm{cm})$ & 25.739 & 34.104 & 39.788 & 91.444 & 85.993 \\
\hline Power $\left(\mu \mathrm{W} / \mathrm{cm}^{2}\right)$ & 1.757 & 3.085 & 4.199 & 22.181 & 19.615 \\
\hline
\end{tabular}

Table 3.3: Vertical polarization, experimental dressing field

Using the above equations for $g, E, E_{d}$, and $P$, we can characterize the dressing field as a function of peak splitting for any case where we can fit the microwave scan to get the center frequencies of the peaks. Table 3.2 and Table 3.3 do this for the case of a horizontally polarized dressing field and a vertically polarized dressing field respectively. Each table contains 5 cases where each scan was repeated equivalently (same initial conditions) under both polarizations so we can draw comparisons 
between them. The scans that are shown in other sections of this chapter have the figure listed at the head of the column.

\begin{tabular}{|c|c|c|c|c|}
\hline Vertical Dressing Pol. & Average & Minimum & Maximum & St. Deviation \\
\hline Splitting $(\mathrm{MHz})\left(\lambda_{1}\right)$ & 15.765 & 8.795 & 27.795 & 9.384 \\
\hline Splitting $(\mathrm{MHz})\left(\lambda_{2}\right)$ & 7.108 & 3.470 & 12.280 & 4.109 \\
\hline $\mathrm{g}$ & 0.376 & 0.269 & 0.434 & 0.063 \\
\hline $\mathrm{E}(\mathrm{mV} / \mathrm{cm})$ & 32.5 & 17.5 & 57.0 & 19.152 \\
\hline$E_{d}(\mathrm{mV} / \mathrm{cm})$ & 34.8 & 19.0 & 61.1 & 20.558 \\
\hline Power $\left(\mu \mathrm{W} / \mathrm{cm}^{2}\right)$ & 4.113 & 0.960 & 9.917 & 4.272 \\
\hline
\end{tabular}

Table 3.4: Horizontal polarization, statistics for $g, E, E_{d}$, and $P$ for Table 3.2

\begin{tabular}{|c|c|c|c|c|}
\hline Horizontal Dressing Pol. & Average & Minimum & Maximum & St. Deviation \\
\hline Splitting $(\mathrm{MHz})\left(\lambda_{1}\right)$ & 21.904 & 9.045 & 37.600 & 12.991 \\
\hline Splitting $(\mathrm{MHz})\left(\lambda_{2}\right)$ & 16.617 & 9.045 & 25.885 & 8.350 \\
\hline $\mathrm{g}$ & 0.112 & 0.000 & 0.191 & 0.077 \\
\hline $\mathrm{E}(\mathrm{mV} / \mathrm{cm})$ & 54.809 & 25.739 & 89.816 & 30.286 \\
\hline$E_{d}(\mathrm{mV} / \mathrm{cm})$ & 55.414 & 25.739 & 91.444 & 30.871 \\
\hline Power $\left(\mu \mathrm{W} / \mathrm{cm}^{2}\right)$ & 10.167 & 1.757 & 22.181 & 9.875 \\
\hline
\end{tabular}

Table 3.5: Vertical polarization, statistics for $g, E, E_{d}$, and $P$ for Table 3.3

Tables 3.4 and 3.5 show statistics for $g, E, E_{d}$, and $P$ for Table 3.2 and Table 3.3 respectively. They list the average, minimum, maximum, and standard deviation over 5 sample scans each.

The ratio of elliptical components $g$ is not dependent on the field magnitude. This makes it a good number to compare across different cases. The field is more elliptically polarized for a vertically polarized field - $g$ averages 0.38 as opposed to 0.11 . This is a consistent difference. However, it should be stated that there were cases of $g \sim 0$ for vertically polarized fields and $g>0.2$ for horizontally polarized fields. They were less common and weren't part of the set of 10 used above, because they weren't part of a set where data with the same initial conditions was taken with both polarizations for the dressing field.

The magnitude of the field $\left(E\right.$ and $\left.E_{d}\right)$ is dependent on the amount of attenuation used and can be tuned over a range of $0-50 \mathrm{~dB}$ or 1-0.003 relative field strength. We can gain two useful pieces of knowledge from calculating them. First, we can learn the order of magnitude of the dressing field for clear splitting - on the order of $20 \mathrm{mV} / \mathrm{cm}$. Secondly, it was found that for the same attenuation there was a weaker field at the MOT for vertically polarized fields - a little over half the magnitude. The vertical field ionization rods scatter the vertically polarized field more strongly, decreasing the 
magnitude of the field at the MOT.

The power of the field $P$ is on the scale of $1 \mu \mathrm{W} / \mathrm{cm}$. Since the output from the passive tripler before the variable attenuator is on the scale of $1 \mathrm{~mW} / \mathrm{cm}$, the calculated number is reasonable.

To get a better idea of the range of magnitude of the dressing field used in the experiment, we look at the Figures 3.12 and 3.13 which show the dressed state splitting over a range of field strengths. First look at the horizontally polarized initial dressing field case, Figure 3.12. This was treated as a linearly polarized case $-\mathrm{g}=0 . E_{d}=2.1 \mathrm{mV} / \mathrm{cm}$ at the earliest point there is discernible splitting and $E_{d}=58.7 \mathrm{mV} / \mathrm{cm}$ for the graph with the largest splitting as the variable attenuator is scanned from $30-5 \mathrm{~dB}$. Next looking at the vertically polarized initial dressing field case (Figure 3.13) $g=0.23$ based on the slope, and the field ranges from $E_{d}=2.6$ to $E_{d}=67.1 \mathrm{mV} / \mathrm{cm}$ when the variable attenuator is scanned from 30-0dB. It was first possible to fit four peaks for $E_{d}=12.2 \mathrm{mV} / \mathrm{cm}$ corresponding to the variable attenuator set at $15 \mathrm{~dB}$. In this experiment the attenuation was changed in steps of $5 \mathrm{~dB}$. When the field is attenuated to less than $2 \mathrm{mV} / \mathrm{cm}$ at the MOT, the splitting is too small for the peaks to be fit separately. Correspondingly if the field is more than $67 \mathrm{mV} / \mathrm{cm}$ the peaks become small and harder to detect relative to the noise.

To summarize, vertically polarized dressing fields tend to result in more elliptical polarization and lower field power compared horizontally polarized fields. The ratio of the elliptical components $g$ ranges from 0-0.43 and the dressing field can be tuned over $E_{d}=2$ to $E_{d}=67 \mathrm{mV} / \mathrm{cm}$. 


\section{Chapter 4}

\section{Microwave Scattering Theory}

\subsection{Modeling the Microwave EM Field Scattering from the Field Ionization Rods}

The field emitted from the microwave horns is assumed to be linear in polarization and constant in magnitude. However, the field that reaches the MOT is affected by a non-negligible amount of scattering from the four rods that provide the field ionization pulse. The goal of this section is to model the electric field over the finite volume of the MOT at the center of four cylindrical rods.

For this purpose, we shall first develop the cylindrical wave functions used to model the scattering waves from a cylinder (Section 4.2). Next we shall model an EM plane wave scattered from one cylinder (Section 4.3). Then we will sum the scattered fields from four rods (each placed at the corner of a square) to get a first order approximation of the field (Section 4.4). In all cases, we will solve for on axis field propagation and small deviations in angle of initial propagation. Once we have the field at the center of the MOT, we can calculate the field over the finite volume of the MOT. This will give an idea of polarization of the field, of how much the field amplitude and polarization vary over the size of the MOT, and the broadening of the observed frequency peaks. Elliptical polarization causes additional splitting in the dressed state spectrum. The amplitude of the field is related to the amount of splitting between the peaks of the dressed states (Section 3.2). When the amplitude of the field varies over the size of the MOT, the atoms at a given position 
in the MOT see a different field and therefore exhibit a different dressed state splitting. When all locations in the MOT are summed over the result is a broadened peak where the magnitude of the broadening depends on the amount of variation in the field.

\subsection{Cylindrical Wave Equations}

The tradition method for representing electromagnetic fields and solving Maxwell's equations is with a scalar and vector potential. However, Hertz showed that an electromagnetic field could be represented with only a vector potential [11]. This vector is referred to as the Hertz vector or a polarization potential and represented by $\vec{\Pi}$. It must satisfy [11]:

$$
\vec{\nabla}^{2} \vec{\Pi}-\mu \epsilon \frac{\partial^{2} \vec{\Pi}}{\partial t^{2}}=0
$$

where $\mu$ is permeability of the medium, $\epsilon$ the permittivity of the medium, and they are related by the speed of light $c=1 / \sqrt{\mu \epsilon}$. Also, $\vec{\nabla}^{2}=\vec{\nabla} \cdot \vec{\nabla}$ is understood to operate on the rectangular components of $\vec{\Pi}[11]$. Equivalently stated, each component of $\vec{\Pi}$ must satisfy the scalar wave equation.

Next we define the Hertz vector within the cylindrical coordinate system defined by an infinitesimal line element:

$$
d s=\hat{\rho} d \rho+\hat{\phi} \rho d \phi+\hat{z} d z
$$

We can define a $\vec{\Pi}$ directed along the z-axis $\left(\Pi_{\rho}=\Pi_{\phi}=0, \Pi_{z} \neq 0\right)$ where $\Pi_{z}$ can be represented by the elementary harmonic solutions:

$$
\vec{\Pi}=\hat{z} \Pi_{z}=\hat{z} \psi(\rho, \phi) e^{ \pm i k_{z} z+i \omega t}
$$

where $\Pi_{z}$ satisfies the scalar wave equation 4.1. We can calculate the field associated with $\vec{\Pi}$ as:

$$
\overrightarrow{E^{(1)}}=\frac{1}{k} \vec{\nabla} \times \vec{\nabla} \times \vec{\Pi}=\vec{\nabla} \times \vec{\nabla} \times\left[\hat{z} \Pi_{z}\right]
$$

where $\overrightarrow{E^{(1)}}$ is the associated electric field. This field can be referred to as the transverse magnetic 
field.

A similar partial field can be derived from a second Hertz vector $\vec{\Pi}^{*} . \vec{\Pi}^{*}=\hat{z} \Pi_{z}^{*}$ is also directed along the z-axis and $\Pi_{z}^{*}$ satisfies the scalar wave equation. The fields associated with it are:

$$
\overrightarrow{E^{(2)}}=\vec{\nabla} \times\left[\hat{z} \Pi_{z}^{*}\right]
$$

This field can be referred to as a "transverse electric" field and is perpendicular to $\overrightarrow{E^{(1)}}$. Both fields have corresponding magnetic components that will not be dealt with here.

The superposition of these fields $\left(\overrightarrow{E^{(1)}}+\overrightarrow{E^{(2)}}\right)$ is general enough to represent any field with boundary conditions parallel to the z-axis [11]. Our problem's boundary condition is a cylindrical rod parallel to and centered on the z-axis. Therefore, despite the fact our field doesn't propagate in

the $\hat{z}$ direction, we can use the above definitions for $\overrightarrow{E^{(1)}}$ and $\overrightarrow{E^{(2)}}$ to derive our cylindrical vector field equations. To do this we must first solve the scalar wave equation in cylindrical coordinates. Once we have those solutions, we can substitute them into equations 4.4 and 4.5 to find a transverse electric and transverse magnetic vector wave functions. In all further derivations we will solve for the electric component of the field as that is what is used to calculate the dressed state matrix elements in Section 3.2. The magnetic component associated with the electromagnetic wave is ignored.

\subsubsection{Scalar Wave Equation in Cylindrical Coordinates}

The electric polarization potentials $\Pi_{z}$ and $\Pi_{z}^{*}$ must satisfy the scalar wave equation [11]:

$$
\vec{\nabla}^{2} \psi-\mu \epsilon \frac{\partial^{2} \psi}{\partial t^{2}}=0
$$

where $\psi$ refers to both $\Pi_{z}$ and $\Pi_{z}^{*}$. $\Pi_{z}$ and $\Pi_{z}^{*}$ are defined in terms of equation 4.3. It is possible to separate the components and write it as:

$$
\psi(\vec{r}, t)=P(\rho) \Phi(\phi) e^{i k_{z} z+i \omega t}
$$

where $k$ and $\omega$ are related by:

$$
c=\frac{\omega}{k}
$$


and $\vec{k}$ components are defined by the equation below and diagrammed in Figure 4.1.

$$
\begin{aligned}
|\vec{k}|^{2}=k^{2} & =k_{\rho}^{2}+k_{z}^{2} \\
k_{\rho} & =k \sin \theta_{k} \\
k_{z} & =k \cos \theta_{k} \\
k_{x} & =k_{\rho} \cos \phi_{k} \\
k_{y} & =k_{\rho} \sin \phi_{k}
\end{aligned}
$$

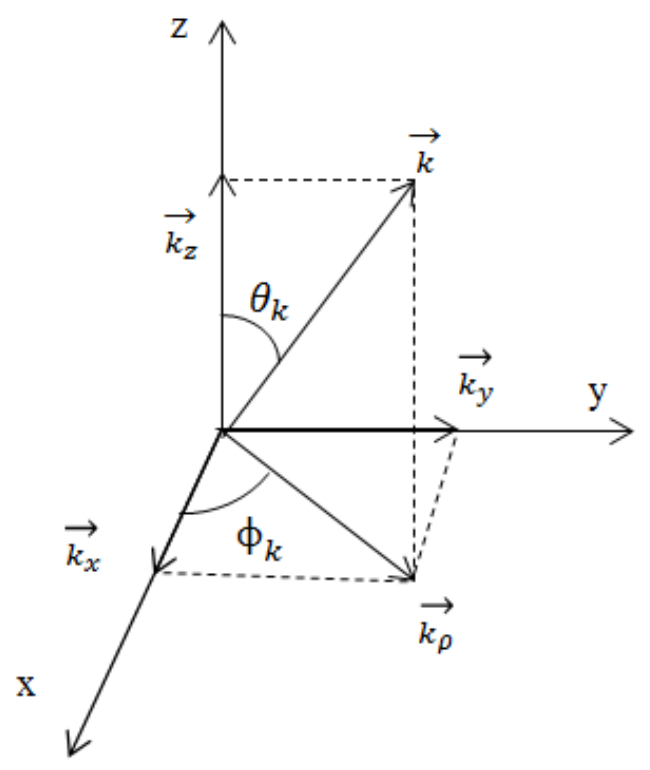

Figure 4.1: Diagram of $\mathrm{k}$ and its components.

The cylindrical coordinate scalar wave equation is:

$$
\begin{aligned}
{\left[\frac{1}{\rho} \frac{\partial}{\partial \rho} \rho \frac{\partial}{\partial \rho}+\frac{1}{\rho^{2}} \frac{\partial^{2}}{\partial \phi^{2}}+\frac{\partial^{2}}{\partial z^{2}}-\mu \epsilon \frac{\partial^{2}}{\partial t^{2}}\right] \psi } & =0 \\
=\frac{1}{\rho} \frac{\partial}{\partial \rho} \rho \frac{\partial P}{\partial \rho} \Phi+\frac{1}{\rho^{2}} \frac{\partial^{2} \Phi}{\partial \phi^{2}} P+\left[-k_{z}^{2}+k^{2}\right] P \Phi & =0
\end{aligned}
$$


Inserting $\psi$ defined in equation 4.27 gives:

$$
\frac{1}{\rho} \frac{\partial}{\partial \rho} \rho \frac{\partial P}{\partial \rho} \Phi+\frac{1}{\rho^{2}} \frac{\partial^{2} \Phi}{\partial \phi^{2}} P+\left[-k_{z}^{2}+k^{2}\right] P \Phi=0
$$

This can be separated in terms of $\rho$ and $\phi$ :

$$
\frac{\rho}{P(\rho)} \frac{\partial}{\partial \rho} \rho \frac{\partial P(\rho)}{\partial \rho}+\rho^{2} k_{\rho}^{2}=\frac{1}{\Phi(\phi)} \frac{\partial^{2} \Phi(\phi)}{\partial \phi^{2}}
$$

This gives $P(\rho)$ and $\Phi(\phi)$ as the arbitrary solutions of the ordinary differential equations 4.13 and 4.14. [11]

$$
\rho \frac{\partial}{\partial \rho}\left(\rho \frac{\partial P}{\partial \rho}\right)+\left[k_{\rho}^{2} \rho^{2}-n^{2}\right] P=0
$$

$$
\frac{\partial^{2} \Phi}{\partial \phi^{2}}+n^{2} \Phi=0
$$

where $n^{2}$ is the separation constant which gives equations 4.13 and 4.14. Provided inhomogeneities and discontinues of the medium are excluded, the field will be periodic with respect to $\phi$ and $\mathrm{n}$ must be an integer, giving the solution to an $\Phi(\phi)=e^{i n \phi}$. Equation 4.13 can be converted to Bessel's equation 4.16; the solutions for which are separated into four kinds of Bessel functions. We will denote any particular solution of equation 4.13 as $P(\rho)=Z_{n}\left(k_{\rho} \rho\right)$.

As a result, particular solutions of the wave equation periodic in $t$ and $\phi$ con be constructed from elementary waves of this form:

$$
\psi_{n}(\vec{r})=Z_{n}\left(k_{\rho} \rho\right) e^{i n \phi} e^{ \pm i k_{z} z+i \omega t}
$$

\subsubsection{Bessel Functions}

This section will contain a brief review of the kinds of Bessel Functions and some of their properties. More details on Bessel's functions can be found in other works such as Abramowitz and Stegun's 
Handbook of Mathematical Functions [12] and Stratton's Electromagnetic Theory [11].

Let $x=k_{\rho} \rho$. Then equation 4.13 can be rewritten as Bessel's equation [11]:

$$
x^{2} \frac{d^{2} Z_{n}}{d x^{2}}+x \frac{d Z_{n}}{d x}+\left(x^{2}-n^{2}\right)=0
$$

The Bessel function of the first kind is a particular solution which is finite at $x=0$ (i.e. regular). This makes it particularly useful for defining infinite plane waves, as will be demonstrated later. For integer values of $\mathrm{n}$ and for any value of $\mathrm{x}$, both real and complex, we have[12]:

$$
J_{n}(x)=\sum_{m=0}^{\infty} \frac{-1^{m}}{m !(n+m) !}\left(\frac{x}{2}\right)^{n+2 m}
$$

The Bessel function of the second kind is defined by the relation[12]:

$$
Y_{n}(x)=\frac{1}{\sin n \pi}\left[J_{n}(x) \cos n \pi-J_{-n}(x)\right]
$$

It is infinite at $x=0$ (i.e. irregular). This is a very important difference from $J_{n}$ which is regular at $x=0$.

It is possible to approximate the Bessel functions in the limiting case of large $\mathrm{x}(|x| \gg 1,|x| \gg$ $|n|)$. This is useful for understanding the nature of the functions. In this limit, the first and second kind of Bessel functions are[12]:

$$
J_{n}(x) \simeq \sqrt{\frac{2}{\pi x}} \cos \left(x-\frac{2 n+1}{4} \pi\right)
$$

and

$$
Y_{n}(x) \simeq \sqrt{\frac{2}{\pi x}} \sin \left(x-\frac{2 n+1}{4} \pi\right)
$$

At large distances from the origin, the first and second kinds of Bessel's functions correspond to cosine and sine waves respectively. Both are attenuated by a factor of $\sqrt{1 / x}$. This makes them useful for representing standing cylindrical waves. In the same fashion that cosines and sines can be combined to create exponents, $J_{n}$ and $Y_{n}$ can be combined to create Bessel functions of the 
third kind (a.k.a. Hankel Functions of the first and second order) which are useful for representing traveling cylindrical waves.

These are defined by the relations [12]:

$$
H_{n}^{(1)}(x)=J_{n}(x)+i Y_{n}(x)
$$

$$
H_{n}^{(2)}(x)=J_{n}(x)-i Y_{n}(x)
$$

It follows from equations 4.19 and 4.20 that in the limiting case of large $\mathrm{x}(|x| \gg 1,|x| \gg|n|)[12]$ :

$$
H_{n}^{(1)}(x) \simeq \sqrt{\frac{2}{\pi x}} e^{i\left(x-\frac{n+1}{2} \pi\right)}
$$

and

$$
H_{n}^{(2)}(x) \simeq \sqrt{\frac{2}{\pi x}} e^{-i\left(x-\frac{n+1}{2} \pi\right)}
$$

Some useful properties of Bessel functions used in the rest of this chapter[12]:

$$
\begin{gathered}
J_{-n}(x)=(-1)^{n} J_{n}(x) \\
\frac{\partial J_{n}(x)}{\partial x}=J_{n}^{\prime}(x)=\left(J_{n-1}(x)-J_{n+1}(x)\right) \frac{1}{2} \\
=\frac{n}{x} J_{n}(x)-J_{n+1}(x) \\
J_{-n}^{\prime}(x)=(-1)^{n} J_{n}^{\prime}(x) \\
\frac{\partial H_{n}^{(1)}(x)}{\partial x}=H_{n}^{(1)^{\prime}}(x)=\left(H_{n-1}^{(1)}(x)-H_{n+1}^{(1)}(x)\right) \frac{1}{2} \\
=\frac{n}{x} H_{n}^{(1)}(x)-H_{n+1}^{(1)}(x) \\
H_{-n}^{(1)^{\prime}}(x)=e^{i n \pi} H_{n}^{(1)^{\prime}}(x)
\end{gathered}
$$




\subsubsection{Cylindrical Scalar Fields}

The result from the derivations in the previous section is that within an isotropic, homogeneous domain, any scalar field can be represented by:

$$
\psi\left(k_{\rho}, k_{z}, \vec{r}, t\right)=\sum_{n=-\infty}^{\infty} \psi_{n}\left(k_{\rho}, k_{z}, \vec{r}, t\right)
$$

where

$$
\left.\psi_{n}\left(k_{\rho}, k_{z}, \vec{r}, t\right)=e^{i n \phi} Z_{n}^{(} k_{\rho} \rho\right) e^{ \pm i k_{z} z-i \omega t}
$$

where $Z_{n}=J_{n}$ in cases where the field is finite at $\rho=0$. Since it is regular at the origin, this case can be denoted by a $R g \psi$. In cases were the field does not need to be finite at the origin,

$Z_{n}=H_{n}^{(1)}$, denoted by $\operatorname{Irg} \psi \cdot H^{(1)}$ and not $H^{(1)}$ is used because $H^{(1)}$ reduces to a wave traveling radially outward (refer to equation 4.23).

This is similar to the Coulomb equation for the radial functions. There are regular and irregular solutions. Only the regular solutions are used for hydrogen since the radial function goes to zero at the nucleus in that case. Another case where the result is a linear combination of Bessel functions is frequency modulation. Equation 4.29 is an identity used for both frequency modulation calculations and in finding a plane wave specific equation.

$$
e^{i A \cos (\omega t)}=\sum_{n=-\infty}^{\infty} J_{n}(A) e^{i n \omega t+i n \pi / 2}
$$

\subsubsection{Vector Cylindrical Wave Functions}

Equation 4.28 satisfies the scalar wave equation. This gives us our $\Pi_{z}$ and $\Pi_{z}^{*}$ outlined in Section 4.2. To get the actual field we apply equations 4.4 and 4.5. Those are defined as [13]:

$$
E^{(1)}=\vec{N}_{n}\left(k_{\rho}, k_{z}, \vec{r}\right)=\frac{1}{k} \nabla \times \nabla \times\left[\hat{z} \psi_{n}\left(k_{\rho}, k_{z}, \vec{r}\right)\right]
$$




$$
E^{(2)}=\vec{M}_{n}\left(k_{\rho}, k_{z}, \vec{r}\right)=\nabla \times\left[\hat{z} \psi_{n}\left(k_{\rho}, k_{z}, \vec{r}\right)\right]
$$

Using $\psi_{n}$ defined in equation 4.28 results in:

$$
\begin{aligned}
& \vec{M}_{n}\left(k_{i \rho}, k_{i z}, \vec{r}\right)=e^{i \omega t+i k_{z} z+i n \phi}\left[\frac{i n}{\rho} Z_{n}\left(k_{\rho} \rho\right) \hat{\rho}-k_{\rho} Z_{n}^{\prime}\left(k_{\rho} \rho\right) \hat{\phi}\right] \\
& \vec{N}_{n}=\frac{1}{k} e^{i \omega t+i k_{z} z+i n \phi}\left[i k_{z} k_{\rho} Z_{n}^{\prime}\left(k_{\rho} \rho\right) \hat{\rho}-\frac{n k_{z}}{\rho} Z_{n}\left(k_{\rho} \rho\right) \hat{\phi}+k_{\rho}^{2} Z_{n}\left(k_{\rho} \rho\right) \hat{z}\right]
\end{aligned}
$$

Note that $Z_{n}=J_{n}$ in the regular case, $Z_{n}=H_{n}^{(1)}$ in the irregular case for outgoing cylindrical

waves, and $Z_{n}=H_{n}^{(2)}$ in the irregular case for incoming cylindrical waves. This gives us a general vector cylindrical wave equation of [13]:

$$
\vec{E}=\sum_{n=-\infty}^{\infty}\left[a_{n}^{(M)} \overrightarrow{M_{n}}\left(k_{\rho}, k_{z}, \vec{r}\right)+a_{n}^{(N)} \overrightarrow{N_{n}}\left(k_{\rho}, k_{z}, \vec{r}\right)\right]
$$

where $a_{n}^{(M)}$ and $a_{n}^{(N)}$ are defined by the specific boundary conditions. The problem of interest will have an incoming plane wave defined in terms of $J_{n}$ in the next section and an outgoing cylindrical wave in terms of $H_{n}^{(1)}$.

\subsubsection{Plane Waves in Cylindrical Wave Functions}

Equation 4.34 is the general form for a cylindrical wave function. It is possible to write a specific equation for an infinite incoming plane wave in cylindrical coordinates. Since a plane wave is finite at any point, including $\rho=0$, it's necessary to use the regular form of the equations with $Z_{n}=J_{n}$. 
Now using the definitions of $k_{x}, k_{y}, x$, and $y$, in terms of cylindrical coordinates [13]:

$$
\begin{aligned}
k_{x} x+k_{y} y & =k_{\rho} \rho \cos \phi_{k} \cos \phi+k_{\rho} \rho \sin \phi_{k} \sin \phi \\
& =k_{r} h o \rho \cos \left(\phi-\phi_{k}\right)
\end{aligned}
$$

Using the same relation used to calculate frequency modulation (equation 4.29) and the definition of $R g \psi_{n}$ (equation 4.28) we get:

$$
\begin{aligned}
e^{i\left(k_{x} x+k_{y} y+k_{z} z\right)} & =e^{i k_{\rho} \rho \cos \left(\phi-\phi_{k}\right)+i k_{z} z} \\
& =\sum_{n=-\infty}^{\infty} J_{n}\left(k_{\rho} \rho\right) e^{i n\left(\phi-\phi_{k}+i n \pi / 2\right)} e^{i k_{z} z} \\
& =\sum_{n=-\infty}^{\infty} R g \psi_{n}\left(k_{\rho}, k_{z}, \vec{r}\right) e^{i n \phi_{k}+i n \pi / 2}
\end{aligned}
$$

If we assume a scalar field propagating in the $\hat{k}$ direction, we can get two vector fields from $\nabla \times \hat{z}$ and $\nabla \times \nabla \times \hat{z}$ First we apply $\nabla \times \hat{z}$ to both sides using the definitions of $k_{z}=k \cos \theta_{k}$ and $k_{\rho}=k \sin \theta_{k}$.

$$
\begin{aligned}
\nabla \times \hat{z} e^{i\left(k_{x} x+k_{y} y+k_{z} z+i \omega t\right)} & =\sum_{n=-\infty}^{\infty} R g \vec{M}_{n}\left(k_{\rho}, k_{z}, \vec{r}\right) e^{i n \phi_{k}+i n \pi / 2} \\
& =i \vec{k} \times \hat{z} e^{i \vec{k} \cdot \vec{r}}=-i k_{\rho} \hat{h}\left(\theta_{k}, \phi_{k}\right) e^{i \vec{k} \cdot \vec{r}}
\end{aligned}
$$

Where $\hat{h}$ is the horizontal polarization vector. Therefore, we can express the horizontal component of the plane wave in terms of a sum over $\operatorname{Rg} \vec{M}_{n}$.

$$
\hat{h}\left(\theta_{k}, \phi_{k}\right) e^{i \vec{k} \cdot \vec{r}}=\frac{i}{k_{\rho}} \sum_{n=-\infty}^{\infty} R g \vec{M}_{n}\left(k_{\rho}, k_{z}, \vec{r}\right) i^{n} e^{i n \phi_{k}}
$$

Taking the curl of both sides leads to the vertical polarization vector:

$$
i \vec{k} \times \hat{h}\left(\theta_{k}, \phi_{k}\right) e^{i \vec{k} \cdot \vec{r}}=\frac{i k}{k_{\rho}} \sum_{n=-\infty}^{\infty} R g N_{n}\left(k_{\rho}, k_{z}, \vec{r}\right) i^{n} e^{i n \phi_{k}}
$$




$$
\hat{v}\left(\theta_{k}, \phi_{k}\right) e^{i \vec{k} \cdot \vec{r}}=\frac{-1}{k_{\rho}} \sum_{n=-\infty}^{\infty} \operatorname{Rg} N_{n}\left(k_{\rho}, k_{z}, \vec{r}\right) i^{n} e^{i n \phi_{k}}
$$

Therefore, the general transformation from a Cartesian plane wave to a cylindrical plane wave is:

$$
\begin{aligned}
\vec{E}_{i}(\vec{r}) & =\left(E_{h i} \hat{h}_{i}+E_{v i} \hat{v}_{i}\right) e^{i \vec{k} \cdot \vec{r}+i \omega t} \\
& =\sum_{n=-\infty}^{\infty} \frac{i^{n} e^{i n \phi_{k}}}{k_{\rho}}\left[i E_{h i} \operatorname{Rg} M_{n}\left(k_{\rho}, k_{z}, \vec{r}\right)-E_{v i} \operatorname{RgN}\left(k_{\rho}, k_{z}, \vec{r}\right)\right]
\end{aligned}
$$

\subsection{Scattering from One Cylinder}

Now that we have covered the general forms of the vector cylindrical waves and the transformation from a Cartesian plane wave to a cylindrical plane wave, we apply these forms to our problem. First we will derive the specific case of scattering an initial electromagnetic plane wave from a conducting cylinder on the z-axis.

For the purpose of this thesis three assumptions are made. The rods are assumed to be of infinite length and a perfect conductors. Both of these are good assumptions as the length is an order of magnitude larger than any other dimensions in the problem and the rods are metal conductors. Our infinite length assumption allows us to assume that the field is independent of $\mathrm{z}$. The perfect

conductor assumption lets us set $\vec{E}_{\text {int }}$ (the electric field inside the conductor) equal to zero. The other two fields in this problem are the initial electric field $\vec{E}_{i}$ (i.e. incident field) and the scattered field $\vec{E}_{s}$. The third assumption is that the initial field emitted by the horn is an infinite plane wave. On the scale of the diameter of the rod, this is an good assumption.

The initial field $\left(\vec{E}_{i}\right)$ that is emitted from the microwave horns has a wavelength set by the microwave synthesizer. The polarization and direction of propagation are determined by the orientation of the microwave horn. Chapter 2 has more details on the setup. For the fields, the polarization and direction of propagation have a large impact on the scattering field. For all calculated fields in this chapter, we will hold constant the total magnitude of the initial field (normalized to 1 for the sake of the calculations) and the field frequency $(\mathrm{f}=59.8966 \mathrm{GHz})$. The polarization and direction 
of propagation vary. They are defined by four initial conditions for each calculation. The first two characterize the field polarization. The polarization has a horizontal component $E_{h i}$ and a vertical component $E_{v i}$ relative to the direction of propagation. The initial field was almost always either solely horizontally polarized or vertically polarized. The direction of propagation is determined by $\hat{k}_{i}$. Its magnitude $k=2 \pi f / c$ is determined by the frequency and is constant. However, the direction it points can be changed by movements of the horn and was likely shifted slightly frequently. $\vec{k}_{i}$ can be defined in terms of spherical coordinates $\left\{k_{i}, \phi_{i}, \theta_{i}\right\}$ as pictured in Figure 4.1 for $\vec{k}=\vec{k}_{i}$. $\theta_{i}$ is the angle between $\vec{k}_{i}$ and the z-axis. $\phi_{i}$ is the angle between $\vec{k}_{i \rho}$ and the $\mathrm{x}$-axis. The field is called on-axis when it is perpendicular to the rods and the square they form. This is defined as the y axis, $=\vec{k}_{i}=\left\{k_{i}, \pi / 2, \pi / 2\right\}$. Our field equations are in terms of cylindrical coordinates $\left\{k_{i \rho}, \phi_{i}, k_{i z}\right\}(\phi$ is defined the same in both systems). Components of $\overrightarrow{k_{i}}$ are used as defined as in equation 4.9 for $\vec{k}=\vec{k}_{i}$.

The general equation for the initial fields is:

$$
\begin{aligned}
\vec{E}_{i} & =e^{i \vec{k} \cdot \vec{r}+i \omega t}\left[E_{h i} \hat{h}+E_{v i} \hat{v}\right] \\
& =\sum_{n=-\infty}^{\infty} \frac{i^{n} e^{-i n \phi_{i}}}{k_{i \rho}}\left[i E_{h i} R g \overrightarrow{M_{n}}\left(k_{i \rho}, k_{i z}, \vec{r}\right)-E_{v i} \operatorname{Rg} \overrightarrow{N_{n}}\left(k_{i \rho}, k_{i z}, \vec{r}\right)\right]
\end{aligned}
$$

defined in equation 4.41 and the scattered field is:

$$
\overrightarrow{E_{s}}=\sum_{n=-\infty}^{\infty} \frac{i^{n} e^{-i n \phi_{i}}}{k_{i \rho}}\left[a_{n}^{(M)} \overrightarrow{M_{n}}\left(k_{i \rho}, k_{i z}, \vec{r}\right)+a_{n}^{(N)} \overrightarrow{N_{n}}\left(k_{i \rho}, k_{i z}, \vec{r}\right)\right]
$$

where $\overrightarrow{M_{n}}$ and $\overrightarrow{N_{n}}$ are defined in equations 4.32 and 4.33 respectively. The coefficients $a_{n}^{(M)}$ and $a_{n}^{(N)}$ are determined by the initial field and can be found using the boundary condition at the surface of the rod defined in equation 4.44. Since the rods are conductors, there is no internal field. $\left(E_{\text {int }}=0\right)$ The radius of the rod is defined as $a=0.825 \mathrm{~mm}$.

$$
\hat{\rho} \times\left(E_{i}(a, \phi, z)+E_{s}(a, \phi, z)\right)=\hat{\rho} \times E_{\text {int }}(a, \phi, z)=0
$$


Inserting in our initial fields generates two equations. One for the $\hat{\phi}$ component and one for the $\hat{z}$ component of the cross product. Equivalently, this means that the $\hat{\phi}$ and $\hat{z}$ components of the total field are zero.

$$
\begin{aligned}
& \frac{i^{n} e^{-i n \phi_{i}}}{k_{i \rho}}\left[-k_{i \rho} J_{n}^{\prime}\left(k_{i \rho} a\right) i E_{h i}-k_{i \rho} H_{n}^{(1)^{\prime}}\left(k_{i \rho} a\right) a_{n}^{(M)}\right. \\
& \left.+E_{v i} \frac{n k_{i z}}{k a} J_{n}\left(k_{i \rho} a\right)-\frac{n k_{i z}}{k a} H_{n}^{(1)}\left(k_{i \rho} a\right) a_{n}^{(N)}\right]=0 \\
& \frac{i^{n} e^{-i n \phi_{i}}}{k_{i \rho}}\left[-\frac{k_{i \rho}^{2}}{k} J_{n}\left(k_{i \rho} a\right) E_{v i}+\frac{k_{i \rho}^{2}}{k} H_{n}^{(1)}\left(k_{\rho i} a\right)\right]=0
\end{aligned}
$$

When solved for any n:

$$
\begin{aligned}
& a_{n}^{(M)}=-i E_{h i} \frac{J_{n}^{\prime}\left(k_{i \rho} a\right)}{H_{n}^{(1)^{\prime}}\left(k_{i \rho} a\right)} \\
& a_{n}^{(N)}=E_{v i} \frac{J_{n}\left(k_{i \rho} a\right)}{H_{n}^{(1)}\left(k_{i \rho} a\right)}
\end{aligned}
$$

Equations 4.47 and 4.48 can be substituted into equation 4.43 to get the equation for the scattered fields. The scattered field arising from the vertical and horizontal initial field components are separable, and doing this makes the equations easier to work with. In addition, the most common cases are either an initial field polarized entirely horizontally or entirely vertically. By separating the two cases, it's easier to detect patterns or symmetries that arise. Equations 4.50 and 4.51 show the fully expanded form of the scattered field.

$$
\overrightarrow{E_{s}}(\vec{r})=\overrightarrow{E_{s, h}}(\vec{r})+\overrightarrow{E_{s, v}}(\vec{r})
$$




$$
\begin{aligned}
\vec{E}_{s, h}(\vec{r})= & \sum_{n=-\infty}^{\infty} \frac{i^{n} e^{-i n \phi_{i}}}{k_{i \rho}} a_{n}^{(M)} \overrightarrow{M_{n}}\left(k_{i \rho}, k_{i z}, \vec{r}\right) \\
= & e^{i \omega t} \sum_{n=-\infty}^{\infty} \frac{i^{-n} e^{i n\left(\phi-\phi_{i}\right)+i k_{i z} z}}{k_{i \rho}}\left(-i E_{h i} \frac{J_{n}^{\prime}\left(k_{i \rho} a\right)}{H_{n}^{(1)^{\prime}}\left(k_{i \rho} a\right)}\right) \\
& {\left[\frac{i n}{\rho} H_{n}^{(1)}\left(k_{i \rho} \rho\right) \hat{\rho}-k_{i \rho} H_{n}^{(1)^{\prime}}\left(k_{i \rho} \rho\right) \hat{\phi}\right] } \\
\vec{E}_{s, v}(\vec{r})= & \sum_{n=-\infty}^{\infty} \frac{i^{n} e^{-i n \phi_{i}}}{k_{i \rho}} a_{n}^{(N)} \vec{N}_{n}\left(k_{i \rho}, k_{i z}, \vec{r}\right) \\
= & e^{i \omega t} \sum_{n=-\infty}^{\infty} \frac{i^{n} e^{i n\left(\phi-\phi_{i}\right)+i k_{i z} z}}{k_{i \rho}} E_{v i}\left(\frac{J_{n}\left(k_{i \rho} a\right)}{H_{n}^{(1)}\left(k_{i \rho} a\right)}\right) \\
& {\left[i k_{i z} k_{i \rho} H_{n}^{(1)^{\prime}}\left(k_{i \rho} \rho\right) \hat{\rho}+H_{n}^{(1)}\left(k_{i \rho} \rho\right)\left(-\frac{n k_{i z}}{\rho} \hat{\phi}+k_{i \rho}^{2} \hat{z}\right)\right] }
\end{aligned}
$$

Both these field equations include a sum from negative infinity to infinity. Due to the relations between $H_{n}^{(1)}$ and $H_{-n}^{(1)}$, it is possible to simplify $\overrightarrow{E_{s}}$ from a sum from negative infinity to infinity to a sum from one to infinity plus the $n=0$ case. This also turns many of the exponentials into cosines and sines which helps make the symmetries of the scattered field more apparent. To do this, let's first solve the $\vec{E}_{s, h}$, initial horizontal field case. Let:

$$
\vec{E}_{s, h}(\vec{r})=\sum_{n=-\infty}^{\infty} \vec{E}_{s, h, n}(\vec{r})
$$

So:

$$
\begin{aligned}
\vec{E}_{s, h,-n}(\vec{r})= & e^{i \omega t} \frac{i^{-n} e^{i(-n)\left(\phi-\phi_{i}\right)+i k_{i z} z}}{k_{i \rho}}\left(-i E_{h i} \frac{J_{-n}^{\prime}\left(k_{i \rho} a\right)}{H_{-n}^{(1)^{\prime}}\left(k_{i \rho} a\right)}\right) \\
& {\left[\frac{i(-n)}{\rho} H_{-n}^{(1)}\left(k_{i \rho} \rho\right) \hat{\rho}-k_{i \rho} H_{-n}^{(1)^{\prime}}\left(k_{i \rho} \rho\right) \hat{\phi}\right] }
\end{aligned}
$$

To simplify equation 4.53 , the following relation and the relations in equation 4.25 and 4.26 are 
used.

$$
i^{-n}=(-1)^{n} * i^{n}
$$

Giving:

$$
\begin{aligned}
\vec{E}_{s, h,-n}(\vec{r})= & e^{i \omega t} \frac{(-1)^{n} i^{n} * e^{-i n\left(\phi-\phi_{i}\right)+i k_{i z} z}}{k_{i \rho}}\left(-i E_{h i} \frac{J_{n}^{\prime}\left(k_{i \rho} a\right)}{H_{n}^{(1)^{\prime}}\left(k_{i \rho} a\right)}\right) \\
& {\left[\frac{i(-n)}{\rho}(-1)^{n} H_{n}^{(1)}\left(k_{i \rho} \rho\right) \hat{\rho}-k_{i \rho}(-1)^{n} H_{n}^{(1)^{\prime}}\left(k_{i \rho} \rho\right) \hat{\phi}\right] } \\
= & e^{i \omega t}(-1)^{2 n} e^{-i n\left(\phi-\phi_{i}\right)} \frac{i^{n} * e^{i k_{i z} z}}{k_{i \rho}}\left(-i E_{h i} \frac{J_{n}^{\prime}\left(k_{i \rho} a\right)}{H_{n}^{(1)^{\prime}}\left(k_{i \rho} a\right)}\right) \\
& {\left[\frac{-i n}{\rho} H_{n}^{(1)}\left(k_{i \rho} \rho\right) \hat{\rho}-k_{i \rho} H_{n}^{(1)^{\prime}}\left(k_{i \rho} \rho\right) \hat{\phi}\right] } \\
= & e^{i \omega t} e^{-i n\left(\phi-\phi_{i}\right)} \frac{i^{n} * e^{i k_{i z} z}}{k_{i \rho}}\left(-i E_{h i} \frac{J_{n}^{\prime}\left(k_{i \rho} a\right)}{H_{n}^{(1)^{\prime}}\left(k_{i \rho} a\right)}\right) \\
& {\left[\frac{-i n}{\rho} H_{n}^{(1)}\left(k_{i \rho} \rho\right) \hat{\rho}-k_{i \rho} H_{n}^{(1)^{\prime}}\left(k_{i \rho} \rho\right) \hat{\phi}\right] }
\end{aligned}
$$

Adding $\vec{E}_{s, h,-n}(\vec{r})$ and $\vec{E}_{s, h, n}(\vec{r})$ gives:

$$
\begin{aligned}
\vec{E}_{s, h,-n}(\vec{r})+ & \vec{E}_{s, h, n}(\vec{r})=\frac{e^{i \omega t} i^{n} * e^{i k_{i z} z}}{k_{i \rho}}\left(-i E_{h i} \frac{J_{n}^{\prime}\left(k_{i \rho} a\right)}{H_{n}^{(1)^{\prime}}\left(k_{i \rho} a\right)}\right) \\
& \left(\left[-e^{-i n\left(\phi-\phi_{i}\right)}+e^{i n\left(\phi-\phi_{i}\right)}\right] \frac{i n}{\rho} H_{n}^{(1)}\left(k_{i \rho} \rho\right) \hat{\rho}\right. \\
& \left.-\left[e^{-i n\left(\phi-\phi_{i}\right)}+e^{i n\left(\phi-\phi_{i}\right)}\right] k_{i \rho} H_{n}^{(1)^{\prime}}\left(k_{i \rho} \rho\right) \hat{\phi}\right) \\
= & \frac{e^{i \omega t} i^{n} * e^{i k_{i z} z}}{k_{i \rho}}\left(-i E_{h i} \frac{J_{n}^{\prime}\left(k_{i \rho} a\right)}{H_{n}^{(1)^{\prime}}\left(k_{i \rho} a\right)}\right) \\
& {\left[2 \sin \left[n\left(\phi-\phi_{i}\right)\right] \frac{i n}{\rho} H_{n}^{(1)}\left(k_{i \rho} \rho\right) \hat{\rho}-2 \cos \left[n\left(\phi-\phi_{i}\right)\right] k_{i \rho} H_{n}^{(1)^{\prime}}\left(k_{i \rho} \rho\right) \hat{\phi}\right] }
\end{aligned}
$$


Therefore,

$$
\begin{aligned}
\vec{E}_{s, h}(\vec{r})= & e^{i \omega t} \sum_{n=1}^{\infty} \frac{i^{n} e^{i k_{i z} z}}{k_{i \rho}}\left(-i E_{h i} \frac{J_{n}^{\prime}\left(k_{i \rho} a\right)}{H_{n}^{(1)^{\prime}}\left(k_{i \rho} a\right)}\right) \\
& {\left[2 \sin \left[n\left(\phi-\phi_{i}\right)\right] \frac{i n}{\rho} H_{n}^{(1)}\left(k_{i \rho} \rho\right) \hat{\rho}-2 \cos \left[n\left(\phi-\phi_{i}\right)\right] k_{i \rho} H_{n}^{(1)^{\prime}}\left(k_{i \rho} \rho\right) \hat{\phi}\right] } \\
& +i e^{i k_{i z} z} E_{h i} \frac{J_{0}^{\prime}\left(k_{i \rho} a\right)}{H_{0}^{(1)^{\prime}}\left(k_{i \rho} a\right)} H_{0}^{(1)^{\prime}}\left(k_{i \rho} \rho\right) \hat{\phi}
\end{aligned}
$$

An equivalent treatment to $\vec{E}_{s, v}(\vec{r})$ gives equation 4.58 .

$$
\begin{aligned}
\vec{E}_{s, v}(\vec{r})= & e^{i \omega t} \sum_{n=1}^{\infty} \frac{i^{n} e^{i k_{i z} z}}{k k_{i \rho}}\left(E_{v i} \frac{J_{n}\left(k_{i \rho} a\right)}{H_{n}^{(1)}\left(k_{i \rho} a\right)}\right) \\
& {\left[i k_{i z} k_{i \rho} 2 \cos \left[n\left(\phi-\phi_{i}\right)\right] H_{n}^{(1)^{\prime}}\left(k_{i \rho} \rho\right) \hat{\rho}\right.} \\
& \left.+H_{n}^{(1)}\left(k_{i \rho} \rho\right)\left[-\frac{n k_{i z}}{\rho} 2 \sin \left[n\left(\phi-\phi_{i}\right)\right] \hat{\phi}+k_{i \rho}^{2} 2 \cos \left[n\left(\phi-\phi_{i}\right)\right] \hat{z}\right]\right] \\
& +\frac{e^{i k_{i z} z}}{k k_{i \rho}}\left(E_{v i} \frac{J_{0}\left(k_{i \rho} a\right)}{H_{0}^{(1)}\left(k_{i \rho} a\right)}\right)\left[i k_{i z} k_{i \rho} 2 H_{0}^{(1)^{\prime}}\left(k_{i \rho} \rho\right) \hat{\rho}+k_{i \rho}^{2} 2 H_{0}^{(1)}\left(k_{i \rho} \rho\right) \hat{z}\right]
\end{aligned}
$$

Now that we have equations for field scattered from a cylindrical rod, it's possible to calculate the field at different points for $t=0$. The points used are diagrammed in Figure 4.2. The points are defined in terms of cylindrical coordinates in Table 4.1. Finally, the fields at each point are calculated under 6 sets of initial conditions. The initial field can be either vertically or horizontally polarized and ideally propagates perpendicular to the rod on the y-axis. However, it's plausible that it is slightly off axis. So we use the 6 sets of initial conditions repeatedly:

1. horizontally polarized, on axis $\left(\theta_{i}=\pi / 2, \phi_{i}=\pi / 2\right)$

2. horizontally polarized, off axis by $\pi / 26$ vertically $\left(\theta_{i}=6 \pi / 13\right)$

3. horizontally polarized, off axis by $\pi / 26$ horizontally $\left(\phi_{i}=6 \pi / 13\right)$

4. vertically polarized, on axis $\left(\theta_{i}=\pi / 2, \phi_{i}=\pi / 2\right)$

5 . vertically polarized, off axis by $\pi / 26$ vertically $\left(\theta_{i}=6 \pi / 13\right)$

6. vertically polarized, off axis by $\pi / 26$ horizontally $\left(\phi_{i}=6 \pi / 13\right)$ 


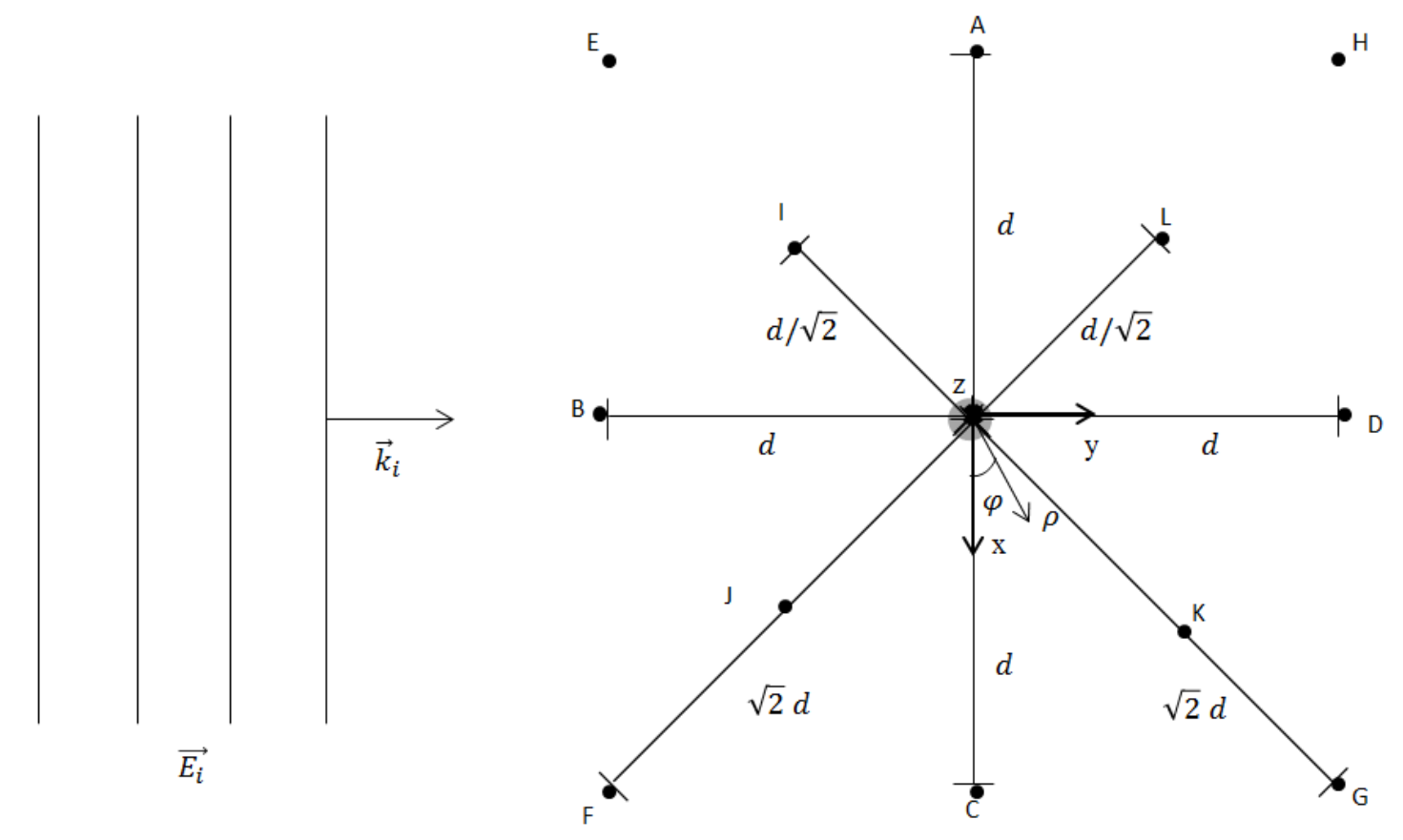

Figure 4.2: Diagram of a cylinder with an incoming plane wave. Points A-H would be positions of other rods relative to another. Points I-L are positions of the MOT relative to a rod

\begin{tabular}{|c|c|}
\hline Point & Coordinates \\
\hline A & $\{\mathrm{d}, \pi, 0\}$ \\
\hline B & $\{\mathrm{d}, 3 \pi / 2,0\}$ \\
\hline C & $\{\mathrm{d}, 0,0\}$ \\
\hline D & $\{\mathrm{d}, \pi / 2,0\}$ \\
\hline E & $\{\sqrt{2} \mathrm{~d}, 5 \pi / 4,0\}$ \\
\hline F & $\{\sqrt{2} \mathrm{~d}, 7 \pi / 4,0\}$ \\
\hline G & $\{\sqrt{2} \mathrm{~d}, \pi / 4,0\}$ \\
\hline H & $\{\sqrt{2} \mathrm{~d}, 3 \pi / 4,0\}$ \\
\hline I & $\{\mathrm{d} / \sqrt{2}, 5 \pi / 4,0\}$ \\
\hline J & $\{\mathrm{d} / \sqrt{2}, 7 \pi / 4,0\}$ \\
\hline K & $\{\mathrm{d} / \sqrt{2}, \pi / 4,0\}$ \\
\hline $\mathrm{L}$ & $\{\mathrm{d} / \sqrt{2}, 3 \pi / 4,0\}$ \\
\hline
\end{tabular}

Table 4.1: Coordinates for points in Figure 4.2 
From studying the calculated values at the different points and equations 4.57 and 4.58 , several conclusions can be drawn. First considering an initially vertical field, there is no scattered field in the $\mathrm{x}$-y plane unless $k_{z} \neq 0$ (i.e. $\theta_{i} \neq \pi / 2$ ). This means to get elliptically polarized fields from an initially vertical polarization, it must either have a vertical tilt in the propagation or have horizontally polarized light from another source such as scattering from the walls of the vacuum chamber. Also, there exists a mirror symmetry over the y axis $-\overrightarrow{E_{s}}(E)=\overrightarrow{E_{s}}(F)$, etc. This is broken if $\overrightarrow{k_{i}}$ isn't parallel to the y axis (i.e. $\left.\phi_{i} \neq \pi / 2\right)$. This ties to the $\cos \left[n\left(\phi-\phi_{i}\right)\right]$ angular dependence found in equation 4.58 .

Considering the case of a horizontally polarized initial field, there is never any scattered field in the $\hat{z}$ direction. However, any scattered field results in field in the $\hat{\rho}$ and $\hat{\phi}$ (i.e. $\hat{x}$ and $\hat{y}$ ) direction. Provided phases don't line up and fields don't cancel, it's very likely to have some form of elliptically polarized fields somewhere within the MOT's volume. Also, while the $\hat{\rho}$ component is mirror symmetric, the $\hat{\phi}$ component is anti-mirror symmetric. This can be predicted looking at equation 4.57. The $\hat{\rho}$ component has a $\cos \left[n\left(\phi-\phi_{i}\right)\right]$ angle dependence, and the $\hat{\phi}$ component has a $\sin \left[n\left(\phi-\phi_{i}\right)\right]$ angle dependence.

Points A-H are potential positions for other rods. Since time dependence hasn't yet been treated, both the real and imaginary parts of the field are relevant. They are all less than 0.4 in magnitude relative to the initial field amplitude of 1 . This is small enough that we won't consider secondary scattering. The fields calculated in this model are a first order approximation. We ignore multiple scatterings from the rod and scatterings from other sources such as the walls of the vacuum chamber and the optics table.

Points I-L are positions of the center of the MOT relative to different rods. The backscattering field at points I and $\mathrm{J}$ is larger than the forward-scattering field at points $\mathrm{K}$ and $\mathrm{L}$. Therefore, the back two rods will contribute more to the scattered field at the MOT than the front two rods. It's interesting to note that in the horizontally polarized case, the $\hat{\phi}$ components will cancel out at the center of the MOT leaving linear polarization. However, as we will see in Section 4.5 this is not the case once we are no longer at the center of the MOT. 


\subsection{Scattering from Four Cylindrical Rods}

We've calculated the scattering from one cylindrical rod in the previous section. Now it is time to calculate the combined scattering from all four field ionization rods. All the field formulas are calculated using a cylindrical coordinate system that places the origin at the center of the rod. However when summing over four rods, it is convenient to convert the fields into a system of Cartesian coordinates that are defined the same for all fields. $\vec{E}_{\rho}$ and $\vec{E}_{\phi}$ are not oriented the same for the

four rods, however we can define an $\vec{E}_{x}$ and $\vec{E}_{y}$ that are. To that end, the relations in equation 4.59 are used. To calculate the sum of the scattering from the 4 cylindrical rods at a point $\mathrm{p}$ we need to figure out the point's position for the cylindrical coordinate systems with the origin at the center of each rod and calculate the phase difference in the initial plane wave between the rods.

The physical system is made up of four cylindrical rods of radius $a=0.825 \mathrm{~mm}$ that are each at the corner of a square with sides of length $d=17.8 \mathrm{~mm}$. The MOT is at the center of the square. The dressing field microwave horn is approximately $l_{d}=160 \mathrm{~mm}$ away from the MOT and is close to perpendicular to the square of rods. The probe field horn is approximately $l_{p}=280 \mathrm{~mm}$ from the MOT and at an angle of $\phi_{p}=0.27$ radians relative to the dressing field direction of propagation. The MOT itself varies in size, but on average has a radius $r=0.4 \mathrm{~mm}$. As in the one cylinder case, the initial field is a infinite plane wave propagating on the y axis. The direction of propagation is defined in spherical coordinates, $\left\{k, \phi_{i}, \theta_{i}\right\}$ which on the y axis are $\{k, \pi / 2, \pi / 2\}$. Figure 4.3 diagrams this roughly to scale. Figure 4.4 shows the problem we are attempting to solve (with the coordinate system orientation shown on the top). Figure 4.5 shows an off axis initial field $(\vec{k}=\{k, 6 \pi / 13, \pi / 2\})$.

$$
\begin{aligned}
& \hat{x}=\cos \phi \hat{\rho}-\sin \phi \hat{\phi} \\
& \hat{y}=\sin \phi \hat{\rho}+\cos \phi \hat{\phi} \\
& \hat{z}=\hat{z}
\end{aligned}
$$

Equation 4.60 shows where the center of the MOT is in each rod's coordinate system. The phase differences are in equation 4.61. Summing these together in equation 4.62 gives the total scattered field at the center of the MOT. For the total field the scattered field must be added to the initial 


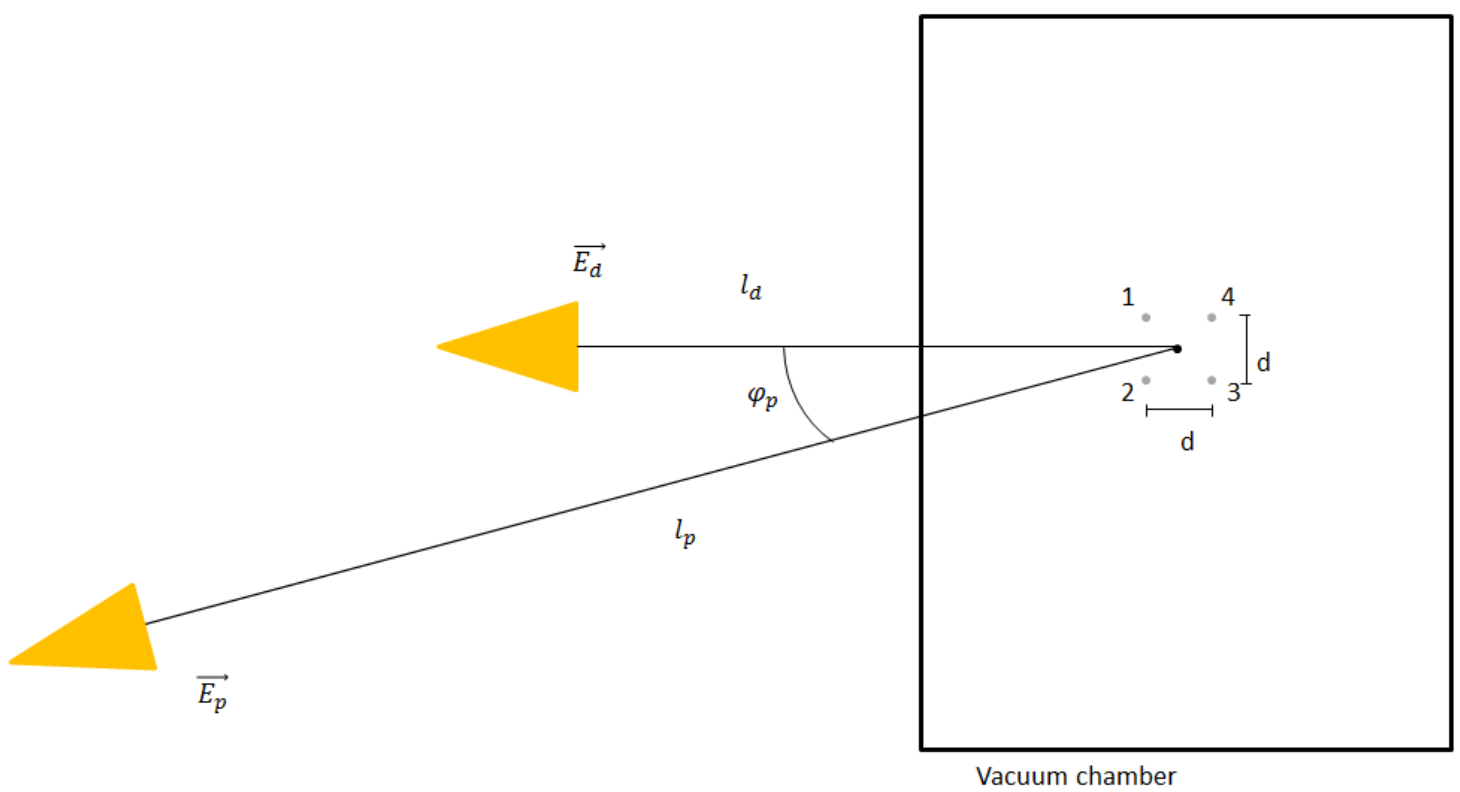

Figure 4.3: Diagram of actual set up drawn roughly to scale

field (equation 4.63) at the center of the MOT, seen in equation 4.64.

$$
\begin{aligned}
& \vec{E}_{s, 1}(M O T)=\vec{E}_{s}(d / \sqrt{2}, \pi / 4,0) * e^{i k \delta_{1}} \\
& \vec{E}_{s, 2}(M O T)=\vec{E}_{s}(d / \sqrt{2}, 3 \pi / 4,0) * e^{i k \delta_{2}} \\
& \vec{E}_{s, 3}(M O T)=\vec{E}_{s}(d / \sqrt{2}, 5 \pi / 4,0) * e^{i k \delta_{3}} \\
& \vec{E}_{s, 4}(M O T)=\vec{E}_{s}(d / \sqrt{2}, 7 \pi / 4,0) * e^{i k \delta_{4}}
\end{aligned}
$$

$$
\begin{aligned}
& \delta_{1}=0 \\
& \delta_{2}=d \cos \phi_{i} \\
& \delta_{3}=\frac{d\left(1+\cos \phi_{i}\right)}{\cos \left(\theta_{i}-\pi / 2\right)} \\
& \delta_{4}=\frac{d}{\cos \left(\theta_{i}-\pi / 2\right)}
\end{aligned}
$$




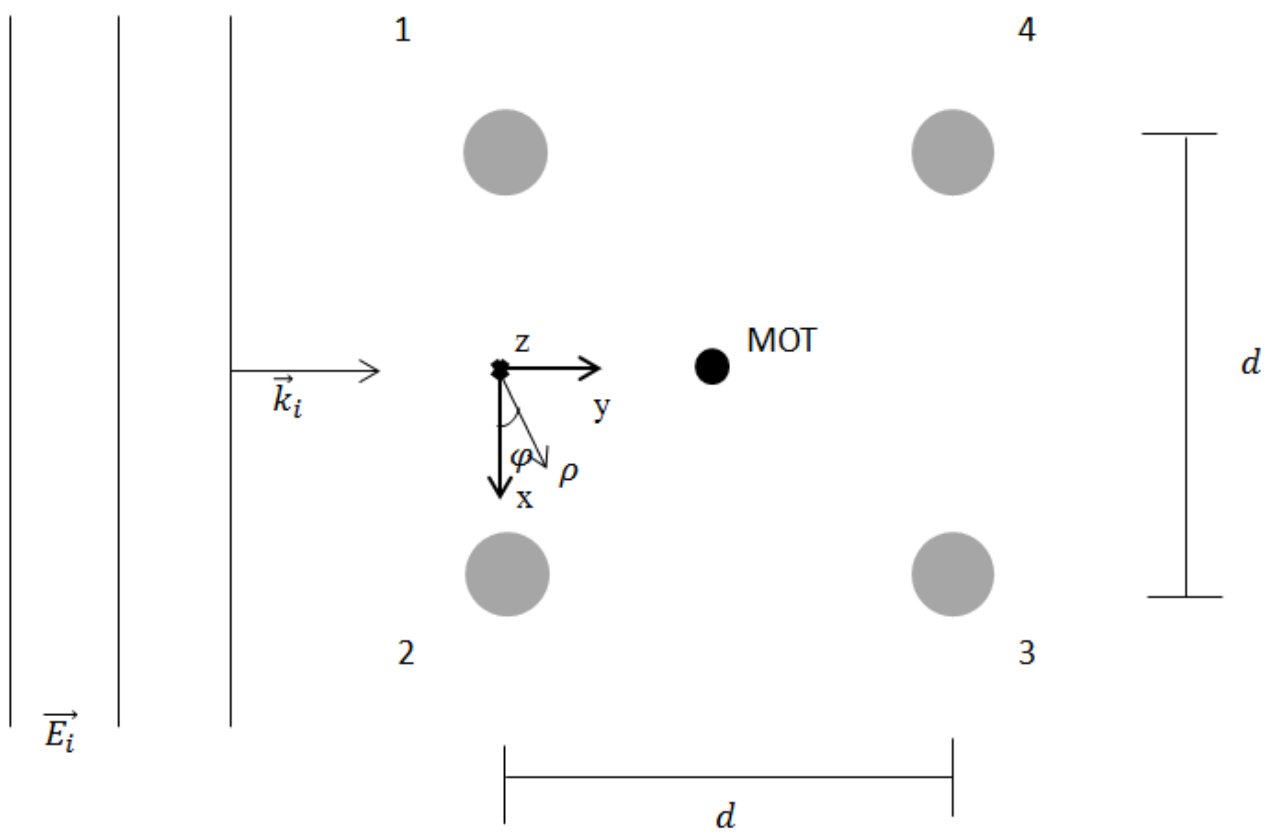

Figure 4.4: Diagram of the 4 field ionization rods with a perpendicular initial field-idealized dressing field, ignoring probe field. 


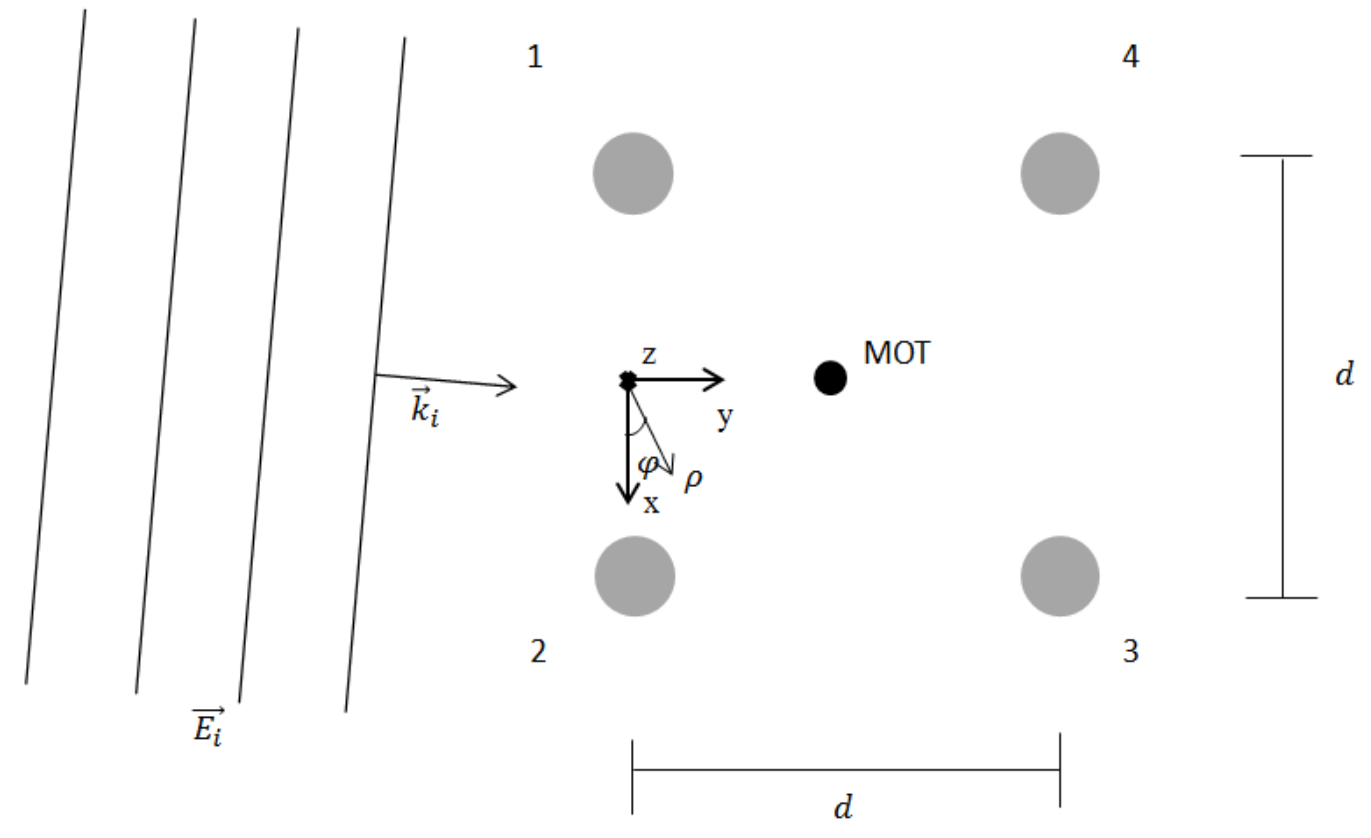

Figure 4.5: Diagram of the 4 field ionization rods with a tilted initial field. 


$$
\begin{aligned}
& \vec{E}_{s, T}(M O T)=\vec{E}_{s, 1}(M O T)+\vec{E}_{s, 2}(M O T)+\vec{E}_{s, 3}(M O T)+\vec{E}_{s, 4}(M O T) \\
& \vec{E}_{i}(M O T)=\vec{E}_{i}(0, d / 2,0)=e^{i k_{y} d / 2+i \omega t}\left[E_{h i} \hat{h}+E_{v i} \hat{v}\right] \\
& \vec{E}_{T}(M O T)=\vec{E}_{s, T}(M O T)+\vec{E}_{i}(0, d / 2,0)
\end{aligned}
$$

The fields calculated have real and imaginary components and a factor of $e^{i \omega t}$ that has been left out. The actual real field at a given point at a given time can be calculated using equation 4.67 if you define the time independent field as in equation 4.65. Inserting in the numbers leads to the calculated fields in Table 4.2 for the 6 sets of initial conditions.

$$
\begin{aligned}
\vec{E}(\vec{r})=\vec{e}(\vec{r})+i \vec{f}(\vec{r}) & \\
\vec{E}_{\text {real }}(\vec{r}, t) & =\operatorname{Re}\left[[\vec{e}(\vec{r})+i \vec{f}(\vec{r})] e^{i \omega t}\right] \\
& =\vec{e}(\vec{r}) \cos (\omega t)-\vec{f}(\vec{r}) \sin (\omega t)
\end{aligned}
$$

where

$$
\begin{aligned}
\vec{E}_{\text {real }}(\vec{r}, t=0) & =\vec{e}(\vec{r}) \\
\vec{E}_{\text {real }}(\vec{r}, t=\pi / 2 \omega) & =\vec{f}(\vec{r})
\end{aligned}
$$

Looking at Table 4.2 it can be seen that the magnitude of the scattering field ranges from 0.4 (close to half the initial field amplitude) to negligible. Also, all but cases 3 and 5 have linear polarization at the center of the MOT. This is not true for cases 1 and 2 at other positions in the MOT. 


\begin{tabular}{|c|c|c|c|c|c|c|c|}
\hline Case & $\mathbf{E}_{\mathbf{v}}$ & $\mathbf{E}_{\mathbf{h}}$ & $\theta_{\mathbf{i}}$ & $\phi_{\mathbf{i}}$ & Time & $\vec{E}_{\mathbf{s}}($ MOT $)$ & $\vec{E}_{\mathbf{T}}(\mathbf{M O T})$ \\
\hline \multirow[t]{2}{*}{1} & \multirow{2}{*}{0} & \multirow[t]{2}{*}{1} & \multirow[t]{2}{*}{$\pi / 2$} & \multirow[t]{2}{*}{$\pi / 2$} & 0 & $\{-0.194,0,0\}$ & $\{-0.026,0,0\}$ \\
\hline & & & & & $\pi / 2 \omega$ & $\{-0.155,0,0\}$ & $\{-1.140,0,0\}$ \\
\hline \multirow[t]{2}{*}{2} & \multirow[t]{2}{*}{0} & \multirow[t]{2}{*}{1} & \multirow[t]{2}{*}{$6 \pi / 13$} & \multirow[t]{2}{*}{$\pi / 2$} & 0 & $\{-0.171,0,0\}$ & $\{-0.083,0,0\}$ \\
\hline & & & & & $\pi / 2 \omega$ & $\{-0.151,0,0\}$ & $\{-1.147,0,0\}$ \\
\hline \multirow[t]{2}{*}{3} & \multirow[t]{2}{*}{0} & \multirow[t]{2}{*}{1} & \multirow[t]{2}{*}{$\pi / 2$} & \multirow[t]{2}{*}{$6 \pi / 13$} & 0 & $\{0.038,-0.059,0\}$ & $\{0.125,-0.049,0\}$ \\
\hline & & & & & $\pi / 2 \omega$ & $\{-0.020,-0.306,0\}$ & $\{-1.009,-0.426,0\}$ \\
\hline \multirow[t]{2}{*}{4} & \multirow[t]{2}{*}{1} & \multirow[t]{2}{*}{0} & \multirow[t]{2}{*}{$\pi / 2$} & \multirow[t]{2}{*}{$\pi / 2$} & 0 & $\{0,0,-0.414\}$ & $\{0,0,-.246\}$ \\
\hline & & & & & $\pi / 2 \omega$ & $\{0,0,-0.179\}$ & $\{0,0,-1.165\}$ \\
\hline \multirow[t]{2}{*}{5} & \multirow[t]{2}{*}{1} & \multirow[t]{2}{*}{0} & \multirow[t]{2}{*}{$6 \pi / 13$} & \multirow[t]{2}{*}{$\pi / 2$} & 0 & $\{0,0.052,-0.362\}$ & $\{0,0.052,-0.274\}$ \\
\hline & & & & & $\pi / 2 \omega$ & $\{0,-0.039,-0.112\}$ & $0,-0.039,-1.108\}$ \\
\hline \multirow[t]{2}{*}{6} & \multirow[t]{2}{*}{1} & \multirow[t]{2}{*}{0} & \multirow[t]{2}{*}{$\pi / 2$} & \multirow[t]{2}{*}{$6 \pi / 13$} & 0 & $\{0,0,-0.005\}$ & $\{0,0,0.083\}$ \\
\hline & & & & & $\pi / 2 \omega$ & $\{0,0,-0.109\}$ & $\{0,0,-1.105\}$ \\
\hline
\end{tabular}

Table 4.2: Microwave field values in Cartesian coordinates at the center of the MOT for different initial conditions

\subsection{Polarization of the Field across the Finite MOT}

The MOT is not a point at the center of the four rods but a finite volume that is approximately spherical. The size of the MOT varies with exact positioning of the various lasers used to form it, the magnitude and direction of external magnetic fields, and the number of atoms released by the Rubidium getter. On average the MOT has a radius of $\mathrm{r}=0.4 \mathrm{~mm}$. To get an idea of how much the field varies over the MOT, the scattered field and total field were calculated at four points on the edge of the MOT and one at the center of the MOT (Point M). Those points are diagrammed in Figure 4.6. The cylindrical coordinates used to calculate each point are found in equations4.68 though equations 4.72 .

$$
\begin{aligned}
\vec{E}_{s}(M) & =\vec{E}_{s, 1}(d / \sqrt{2}, \pi / 4,0)+\vec{E}_{s, 2}(d / \sqrt{2}, 3 \pi / 4,0) \\
& +\vec{E}_{s, 3}(d / \sqrt{2}, 5 \pi / 4,0)+\vec{E}_{s, 4}(d / \sqrt{2}, 7 \pi / 4,0) \\
\vec{E}_{s}(A) & =\vec{E}_{s, 1}(d / \sqrt{2}-r, \pi / 4,0)+\vec{E}_{s, 2}\left(d_{f}, 3 \pi / 4+\phi_{f}, 0\right) \\
& +\vec{E}_{s, 3}(d / \sqrt{2}+r, 5 \pi / 4,0)+\vec{E}_{s, 4}\left(d_{f}, 7 \pi / 4-\phi_{f}, 0\right)
\end{aligned}
$$




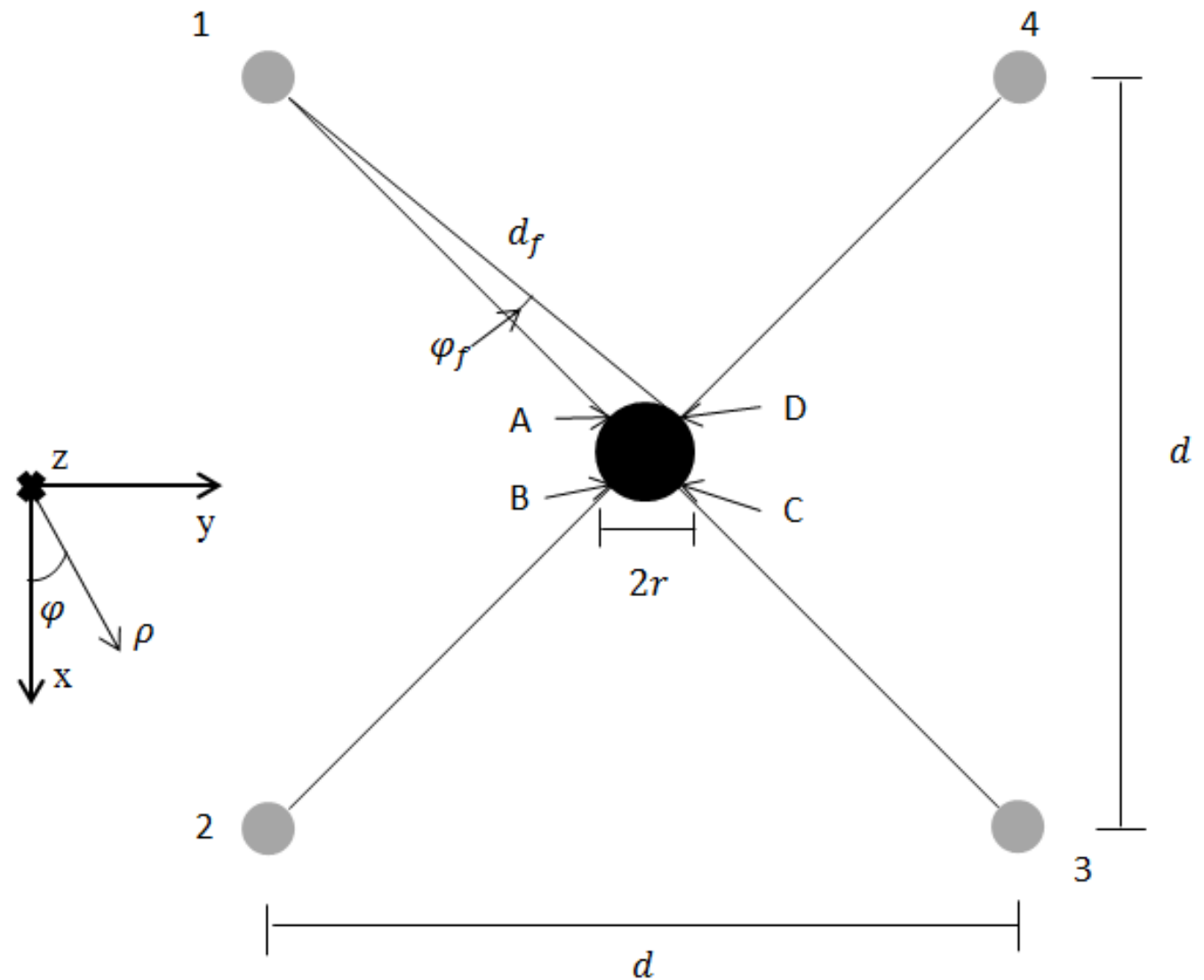

Figure 4.6: Diagram of points measured to get an idea of the variation over the size of the MOT. 


$$
\begin{aligned}
\vec{E}_{s}(B) & =\vec{E}_{s, 1}\left(d_{f}, \pi / 4-\phi_{f}, 0\right)+\vec{E}_{s, 2}(d / \sqrt{2}-r, 3 \pi / 4,0) \\
& +\vec{E}_{s, 3}\left(d_{f}, 5 \pi / 4+\phi_{f}, 0\right)+\vec{E}_{s, 4}(d / \sqrt{2}+r, 7 \pi / 4,0) \\
\vec{E}_{s}(C) & =\vec{E}_{s, 1}(d / \sqrt{2}+r, \pi / 4,0)+\vec{E}_{s, 2}\left(d_{f}, 3 \pi / 4-\phi_{f}, 0\right) \\
& +\vec{E}_{s, 3}(d / \sqrt{2}-r, 5 \pi / 4,0)+\vec{E}_{s, 4}\left(d_{f}, 7 \pi / 4+\phi_{f}, 0\right) \\
& \\
\vec{E}_{s}(D) & =\vec{E}_{s, 1}\left(d_{f}, \pi / 4+\phi_{f}, 0\right)+\vec{E}_{s, 2}(d / \sqrt{2}+r, 3 \pi / 4,0) \\
& +\vec{E}_{s, 3}\left(d_{f}, 5 \pi / 4-\phi_{f}, 0\right)+\vec{E}_{s, 4}(d / \sqrt{2}-r, 7 \pi / 4,0)
\end{aligned}
$$

where:

$$
\begin{aligned}
& d_{f}=\sqrt{r^{2}+\frac{d}{\sqrt{2}}^{2}} \\
& \phi_{f}=\arctan \left(\frac{\sqrt{2} r}{d}\right)
\end{aligned}
$$

Once the field has been calculated at the different points for each of the 6 cases discussed in Table 4.2 it is possible to graph the field for different times to view the polarization. They are graphed over one cycle and are a case of Lissajous figures. Figure 4.7 contains graphs showing the elliptical polarization for cases 1,2,3, and 5 . Cases 4 and 6 are linearly polarized on the $\mathrm{z}$ axis for all positions and were not graphed. The cases are described in Figure 4.7, in Table 4.3, or in Table 4.2.

An elliptically polarized field can be defined in terms of the major and minor axis of the ellipse which we will call $\hat{i}$ and $\hat{j}$ respectively. Note that the orientation of $\hat{i}$ and $\hat{j}$ relative to $\hat{x}, \hat{y}$, and $\hat{z}$ depends on the position and initial field. To be able to compare our calculated scattered field with the experimental results the field is redefined as:

$$
\vec{E}(t)=\left|\vec{E}\left(t_{M A X}\right)\right|[\hat{i} \cos (\omega t)+\hat{j} g \cos (\omega t+\phi)]
$$




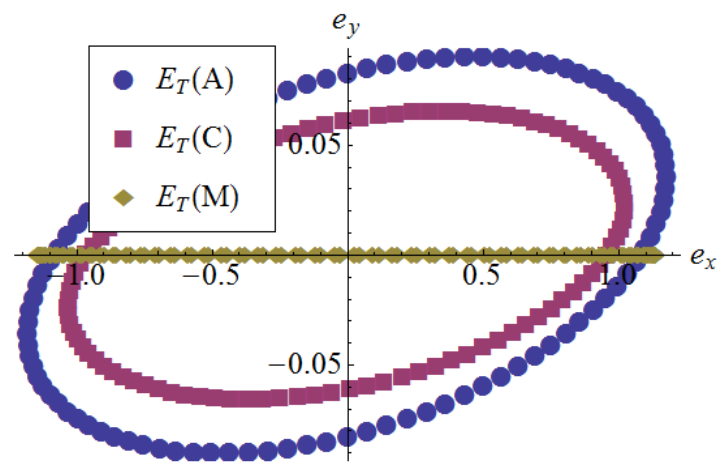

(a) Case 1: Horizontally polarized initial field, on axis.

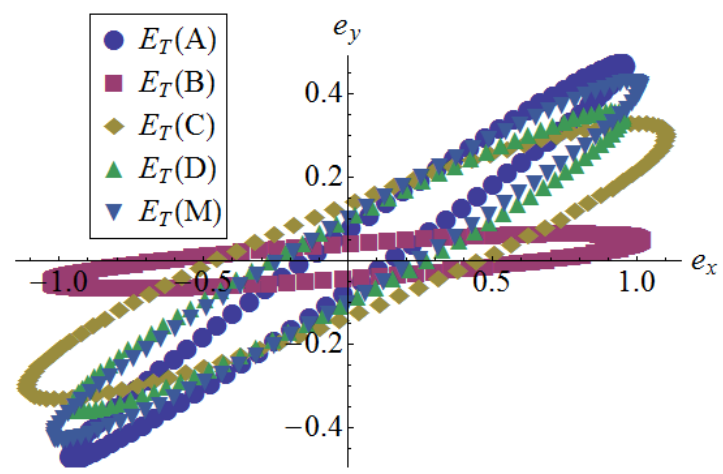

(c) Case3: Horizontally polarized initial field, horizontal tilt.

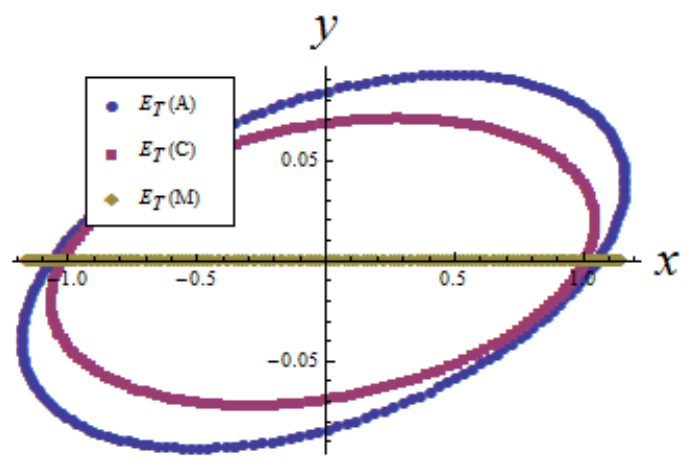

(b) Case 2: Horizontally polarized initial field, vertical tilt.

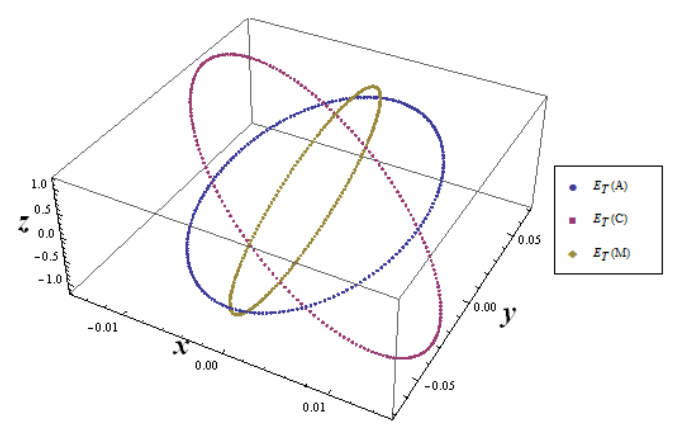

(d) Case 5: Vertically polarized initial field, vertical tilt.

Figure 4.7: These graphs depict the polarization of the field at different positions over a period. 
where $\left|\vec{E}\left(t_{M A X}\right)\right|$ is the maximum field amplitude (i.e. the major axis of the elliptical polarization in the direction $\hat{i})$ which occurs at $t=t_{M A X}+p \pi /(\omega 2)$ where $\mathrm{p}$ is an integer. Equivalently $\left|\vec{E}\left(t_{M I N}\right)\right|$ is the minimum field amplitude and the minor axis of the elliptical polarization in the direction $\hat{j}$. The variable $g$ is defined as the magnitude of the minor axis of the elliptical polarization divided by the major axis of the elliptical polarization in equation 4.75. The variable $\phi$ is the difference in the field's phase between the major and minor polarization axis in equation 4.76.

$$
\begin{aligned}
& g=\left|\vec{E}\left(t_{M I N}\right)\right| /\left|\vec{E}\left(t_{M A X}\right)\right| \\
& \phi=\frac{t_{M I N}-t_{M A X}}{\omega}
\end{aligned}
$$

In Section 3.2, the eigenvalue energies of the dressed state Hamiltonian matrix are developed for a field equivalent to the one defined in equation 4.74 with $\hat{i}=\hat{z}$ and $\hat{j}=\hat{x}$ where $\hat{z}$ and $\hat{x}$ are defined relative to the atom. Since in this chapter $\hat{x}, \hat{y}$, and $\hat{z}$ are used to describe axes relative to the cylindrical rods, we will continue to use $\hat{i}$ and $\hat{j}$. In Section 3.4.1, the eigenvalues from Section 3.2 are used to deduce possible $[g, \phi]$ combinations of the experimental dressing field. Table 4.3 contains the $[g, \phi]$ of the total theoretical field (scattered and initial field) for the 6 different scattering cases discussed in this chapter.

\subsection{Summary}

The vertically polarized field (defined as $\hat{z}$ ) is parallel to the field ionization rods; this causes it to scatter a great deal. However, if the direction of propagation of the field (defined by $\vec{k}$ ) is perfectly perpendicular to the rods (i.e. in the $\hat{y}$ direction), the field scatters only in the $\hat{z}$ polarization. If $\vec{k}$ has a $k_{z}$ component, then there is scattering in the $\hat{x}$ and $\hat{y}$ direction. While the magnitude of the scattered fields is greater for vertically polarized fields, the amount of elliptical polarization (defined by g) was found to be comparable to horizontally polarized light, contrary to the experimental data.

Horizontally polarized light $(\hat{x})$ always scatters into both $\hat{x}$ and $\hat{y}$ polarizations. The summation of the scattering from all 4 rods creates an interference pattern over the volume of the MOT that 


\begin{tabular}{|c|c|c|c|c|c|c|c|}
\hline Case & $\mathbf{E}_{\mathrm{v}}$ & $\mathbf{E}_{\mathbf{h}}$ & $\theta_{\mathbf{i}}$ & $\phi_{\mathbf{i}}$ & Point & $\mathrm{g}$ & $\phi$ \\
\hline \multirow[t]{3}{*}{1} & \multirow[t]{3}{*}{1} & \multirow[t]{3}{*}{0} & \multirow[t]{3}{*}{$\pi / 2$} & \multirow[t]{3}{*}{$\pi / 2$} & $\mathrm{M}$ & 0 & - \\
\hline & & & & & $\mathrm{A}, \mathrm{B}$ & 0.07 & $\pi / 2$ \\
\hline & & & & & C,D & 0.05 & $\pi / 2$ \\
\hline \multirow[t]{3}{*}{2} & \multirow[t]{3}{*}{1} & \multirow[t]{3}{*}{0} & \multirow[t]{3}{*}{$6 \pi / 13$} & \multirow[t]{3}{*}{$\pi / 2$} & $\mathrm{M}$ & 0 & - \\
\hline & & & & & $\mathrm{A}, \mathrm{B}$ & 0.07 & $\pi / 2$ \\
\hline & & & & & C,D & 0.06 & $\pi / 2$ \\
\hline \multirow[t]{5}{*}{3} & \multirow[t]{5}{*}{1} & \multirow[t]{5}{*}{0} & \multirow[t]{5}{*}{$\pi / 2$} & \multirow[t]{5}{*}{$6 \pi / 13$} & $\mathrm{M}$ & 0.08 & $\pi / 2$ \\
\hline & & & & & $\mathrm{A}$ & 0.06 & $\pi / 2$ \\
\hline & & & & & $\mathrm{B}$ & 0.04 & $\pi / 2$ \\
\hline & & & & & $\mathrm{C}$ & 0.11 & $\pi / 2$ \\
\hline & & & & & $\mathrm{D}$ & 0.09 & $\pi / 2$ \\
\hline 4 & 0 & 1 & $\pi / 2$ & $\pi / 2$ & all & 0 & - \\
\hline \multirow[t]{3}{*}{5} & \multirow[t]{3}{*}{0} & \multirow[t]{3}{*}{1} & \multirow[t]{3}{*}{$6 \pi / 13$} & \multirow[t]{3}{*}{$\pi / 2$} & $\mathrm{M}$ & 0.05 & $\pi / 2$ \\
\hline & & & & & $\mathrm{A}, \mathrm{B}$ & 0.07 & $\pi / 2$ \\
\hline & & & & & C,D & 0.03 & $\pi / 2$ \\
\hline 6 & 0 & 1 & $\pi / 2$ & $6 \pi / 13$ & all & 0 & 7 \\
\hline
\end{tabular}

Table 4.3: Summarizes $g=$ ratio of minor axis/major axis and $\phi=$ phase difference between axis of elliptical polarization for the total field at different points in the MOT.

contains elliptically polarized light. Table 4.3 in Section 4.5 lists $[g, \phi]$ for different initial conditions and different positions in the MOT.

In conclusion, the field ionization rods scatter a small amount of field resulting in an elliptical polarization. Scattering from the inner walls of the vacuum chamber and other surfaces probably also cause increase elliptical polarization but are much harder to model. There is likely a complicated standing wave interference pattern over the volume of the MOT. This leads to the graphs seen in the Dressed State Experiment. 


\section{Chapter 5}

\section{Conclusions}

The dressed state was observed to behave as expected according to the theory. Elliptically polarized dressing fields resulting from scattered fields generate four eigenstates instead of the two resulting from linearly polarized dressing fields. The scattered fields result in part from the microwave fields scattering off of the field ionization rods and in part from scattering off other surfaces such as the inside of the vacuum chamber. While it's possible to model the scattering off of the rods, other sources of scattered field are much harder to model. Between that and the difficulty of defining the initial orientation of the field precisely, the field is variable and difficult to define.

Since the scattered fields contribute to elliptical polarization that varies over the area of the MOT, secondary splitting from the elliptical polarization and broadening due to the variance over the finite MOT result. This makes it difficult to identify dipole broadening or any other phenomena as a result of the width of the peaks. In all cases, as the dressing field amplitude was increased, secondary splitting was seen eventually. Initially vertically polarized dressing fields on average resulted in over three times the elliptical component $(g=0.38$ compared to $g=0.11)$ but only a little more than half the total field magnitude $\left(E_{d}=35 \mathrm{mV} / \mathrm{cm}\right.$ compared to $\left.55 \mathrm{mV} / \mathrm{cm}\right)$. The dressing field at the MOT that caused visible splitting ranged from 2.1 to $67.0 \mathrm{mV} / \mathrm{cm}$ controlled by a variable attenuator.

Overall, the dressed state was seen to behave according to well defined theory. We were able to model the scattered field off the rods successfully. Based on the dressed state splitting we can make an estimate of the field magnitude and polarization at the MOT. However, this is definitively not a 
two level system. This makes any careful study we'd like to do more complicated even when all 4 levels are completely separated. 


\section{Bibliography}

[1] T. F. Gallagher. Rydberg Atoms. Cambridge University Press, 1994.

[2] Hyunwook Park. Observation of the dipole-dipole interaction between cold Rydberg atoms by microwave spectroscopy. PhD thesis, Univeristy of Virginia, 2012.

[3] T. Henage L. Isenhower D. D. Yavuz T. G. Walker E. Urban, T. A. Johnson and M. Saffman. Observation of rydberg blockade between two atoms. Nature Phys. 5, 110, 2009.

[4] R. Cote L. M. Duan D. Jaksch J. I. Cirac M. D. Lukin, M. Fleischhauer and P. Zolle. Dipole blockade and quantum information processing in mesoscopic atomic ensembles. Phys. Rev. Lett. 87, 037901, 2001.

[5] T. Wilk A. Chotia M. Viteau D. Comparat P. Pillet A. Browaeys A. Gatan, Y. Miroshnychenko and $\mathrm{Ph}$. Grangier. Observation of collective excitation of two individual atoms in the rydberg blockade regime. Nature Phys. 5, 115, 2009.

[6] William Robert Anderson. Resonant Dipole-Dipole Collisions of Rydberg Atoms in a MagnetoOptical Trap. PhD thesis, University of Virginia, 1996.

[7] Wenhui Li. Probing Dipole-Dipole Interactions in a Frozen Rydberg Gas with Millimeter Waves. $\mathrm{PhD}$ thesis, University of Virginia, 2005.

[8] J. H. Shirley. Solution of the Schroedinger equation with a Hamiltonian periodic in time. Physical Review, 138, 1965.

[9] A. R. Edmonds. Angular Momentum in Quantum Mechanics. Princeton University Press, 1957.

[10] Hans A. Bethe and Edwin E. Salpeter. Quantum mechanics of one-and two-electron atoms. New York :Plenum Pub. Corp., 1977.

[11] J. A. Stratton. Electromagnetic Theory. Wiley - IEEE Press, 2007.

[12] M. Abramowitz and I. A. Stegun. Handbook of Mathematical Functions. National Bureau of Standards Applied Mathematics Series, 1965.

[13] L. Tsang et al. Scattering of Electromagnetic Waves. John Wiley and Sons, Inc., 2000. 